\title{
Article
}

\section{A Phase-Field Perspective on Mereotopology}

\author{
Georg J. Schmitz ${ }^{*}$ \\ ${ }^{1}$ MICRESS group at ACCESS e.V., Intzestr.5, D-52072 Aachen, Germany; \\ *Correspondence: g.j.schmitz@micress.de
}

\begin{abstract}
Mereotopology is a concept rooted in analytical philosophy. The phase-field concept is based on mathematical physics and finds applications in materials engineering. The two concepts seem to be disjoint at a first glance. While mereotopology qualitatively describes static relations between things like $x$ isConnected $y$ (topology) or $x$ isPartOf $y$ (mereology) by first order logic and Boolean algebra, the phase-field concept describes the geometric shape of things and its dynamic evolution by drawing on a scalar field. The geometric shape of any thing is defined by its boundaries to one or more neighboring things. The notion and description of boundaries thus provides a bridge between mereotopology and the phase-field concept. The present article aims to relate phase-field expressions describing boundaries and especially triple junctions to their Boolean counterparts in mereotopology and contact algebra. An introductory overview on mereotopology is followed by an introduction to the phase-field concept already indicating first relations to mereotopology. Mereotopological axioms and definitions are then discussed in detail from a phase-field perspective. A dedicated section introduces and discusses further notions of the isConnected relation emerging from the phase-field perspective like isSpatiallyConnected, isTemporallyConnected, isPhysicallyConnected, isPathConnected and wasConnected. Such relations introduce dynamics and thus physics into mereotopology as transitions from isDisconnected to isPartOf can be described.
\end{abstract}

Keywords: Region based theory of space, RBTS; Contact algebra, Dyadic and Triadic relations, sequent algebra, boundaries, triple junctions, mereotopology, 4D mereotopology, mereophysics, Region Connect Calculus RCC, invariant spacetime interval, Falaco solitons, phase-field method, intuitionistic logic

\section{Introduction}

The term mereology originates from Ancient Greek $\mu$ ćoos (méros, "part") + -logy ("study, discussion, science") while the term topology originates from Ancient Greek тótos (tópos, "place, locality") + -(o)logy ("study of, a branch of knowledge"). The combined expression mereotopology (MT) thus stands for a theory combining mereology (M) and topology $(\mathrm{T})$. The term mereology was first coined by Stanisław Leśniewski as one of three formal systems: protothetic, ontology, and mereology. "Leśniewski was also a radical nominalist: he rejected axiomatic set theory at a time when that theory was in full flower. He pointed to Russell's paradox and the like in support of his rejection, and devised his three formal systems as a concrete alternative to set theory"1. "Parts" in mereology not necessarily have to be spatial parts but may also represent e.g. parts of energy. The top axiom of mereology seems to form a basis to quantify parthood and even to derive a number of physics equations from this philosophical concept [1].

Mereotopology, as philosophical branch, aims at investigating relations between parts and wholes, the connections between parts and the boundaries between them. Mereology and topology are based on primitive relations such as isPartOf or isConnect-

\footnotetext{
${ }^{1}$ https://en.wikipedia.org/wiki/Stanisław_Leśniewski
} 
$e d \mathrm{To}^{2}$, upon which the mereotopology axiomatic systems can be built. An introduction to mereotopology, its fundamental concepts and possible axiomatic systems can be found in the book "Parts \& Places" [2] together with the definition of most of the mereotopological relations and numerous references therein.

Mereotopology thus formalises the description of parthood and connectedness. Mereology maps well onto the hierarchical structure of physical objects like materials enabling to represent materials at different levels of granularity. Any part of a Material is A Material. Any Material hasPart some Material. Any part of a 3DSpace is A 3DSpace. Any part of a Region is $A$ Region. This matches Whitehead's view [3,4] that "points", as well as the other primitive notions in Euclidean geometry like "lines" and "planes" do not have separate existence in reality. As all of them are parts of a 4D-spacetime any of them - from a fundamental perspective - must have a $4 \mathrm{D}$ nature as well. Any (4D) SpaceTimeRegion is $A$ 4DRegion, any 3DVolume is $A$ 4DRegion being "thin" in the time dimension, any 2DPlane is $A$ 4DRegion being "thin" in the time dimension and in one spatial dimension and so forth (see Appendix A). Topology formalises whether space-time regions (3D and/or 4D or even higher dimensional spaces) are connected items or not. In case they are connected, some finite boundary region exists, where they coexist and collocate.

Mereotopology finds application in the development of ontologies. Several foundational ontologies are based on mereotopology as one of the underlying concepts for the specification of relations between individuals and classes, with the most recent example being the Elementary Multiperspective Material Ontology EMMO [5]. Further standardized upper ontologies currently available for use include e.g. BFO [6], BORO method [7], Dublin Core [8], GFO [9], Cyc/OpenCyc/ResearchCyc [10], SUMO [11], UMBEL [12], UFO [13], DOLCE [14,15] and OMT/OPM [16,17].

"In classical Euclidean geometry the notion of "point" is taken as one of the basic primitive notions. In contrast, the region-based theory of space (RBTS) going back to Whitehead [3] and de Laguna [19] has as primitives the more realistic notion of a region as an abstraction of a finite sized physical body, together with some basic relations and operations on regions like the isConnected or isPartOf relations. This is one of the reasons why the extension of mereology complemented by these new relations is commonly called mereotopology "MT". There is no clear difference in the literature between RBTS and mereotopology, and by some authors RBTS is related rather to the so called mereogeometry [20,21], while mereotopology is considered only as a kind of point-free topology, considering mainly topological properties of things. RBTS has applications in computer science because of its more simple way of representing qualitative spatial information. It initiated a special field in Knowledge Representation (KR) [22] called Qualitative Spatial Representation and Reasoning (QSRR) [23]. One of the most popular systems in QSRR is the Region Connection Calculus (RCC) introduced in [24]. The notion of contact algebra (CA) is one of the main tools in RBTS. This notion appears in the literature under different names and formulations as an extension of Boolean algebra with some mereotopological relations [25 - 32]. The simplest system, called just contact algebra (CA) was introduced in [31] as an extension of the Boolean algebra with a binary relation called "contact" and satisfying several simple axioms. Recent work addresses extensions of contact algebra [33].

Most of above approaches are based on monadic relations like Px ( $\mathrm{x}$ is $A$ Part) and dyadic relations like $\mathrm{xCy}(\mathrm{x}$ isConnected $\mathrm{y})$ and thus are limited to relations between two

\footnotetext{
2 throughout this article the CamelCase notation is used for objects/classes while the lowerCamelCase is used for relations

3 section adapted from [18] with slight modifications and amendments
} 
things. In the case of multiple things higher order relations may exist. An example might be a triadic relation like $x$ isConnected $y$ forSome $z$. A simple instance for such a relation would be e.g. a motorway bridge connecting two cities on two sides of a river. If this bridge exists, the cities are connected for a car. Another example is catalysis, where two chemical states $x, y$ are connected (i.e. the reaction occurs) if a catalyst $z$ is present. Else they are disconnected. These simple examples for triadic relations surely need a further formalization. According to Peirce's Reduction Thesis, however, it can be stated that "(a) triads are necessary because genuinely triadic relations cannot be completely analyzed in terms of monadic and dyadic predicates, and (b) triads are sufficient because there are no genuinely tetradic or larger polyadic relations - all higher-arity n-adic relations can be analyzed in terms of triadic and lower-arity relations" ${ }^{4}$. Proofs for Peirce's Reduction Thesis are available e.g. in [34] and [35].

The relation of an n-ary contact is described in a generalization of contact algebra called sequent algebra, which is considered as an extended mereotopology [36, 37]. Sequent algebra replaces the contact between two regions with a binary relation between finite sets of regions and a region satisfying some formal properties of the Tarski consequence relation. Another approach to multiple connected regions is e.g. the Mereology for Connected Structures [38].

Another important aspect not yet covered by classical mereotopology relates to the description of time dependent relations and transitions. In normal language this would refer to the specification of relations like "isConnected", "wasConnected", hasBeenConnected" or similar. 4D-mereotopology [39] specifying e.g. relations like "isHistoricalPartOf", Dynamic Contact Algebra DCA [40, 41], and Dynamic Relational Mereotopology [42] are current first approaches to tackle this challenge.

All above approaches to mereotopology are - to the best of the author's knowledge based on some Boolean algebra and additional relations. They thus only allow for qualitative descriptions like A isConnected B (or isNotConnected as the binary alternative). In contrast, the phase-field approach to mereotopology being depicted in the present article allows the quantitative description of different "degrees of connectivity" ranging e.g. from 0 to $100 \%$. The phase-field perspective thus provides a much higher expressivity and especially allows for describing transitions - e.g. temporal changes - between classes being disjoint in binary, Boolean relations. An example would be a transition from "isDisconnected" via different states of "isConnected" to "isProperPartOf" with a physics example for such a process being a cherry dropping into a region of whipped cream. Eventually also the formulation of relations like "wasConnected", "hasBeenConnected" and many more become possible based on the same approach.

In the spirit of Whitehead's region-based theory of space (RBTS) the regions in the phase-field model are defined by values of the phase-field. The phase-field is a scalar field being defined over a continuous or discretized Euclidian space and thus has some relations with discrete mereotopology DM [43, 44] and with mathematical morphology MM [45]. An essay to describe also this discretized Euclidean spacetime itself based on mereology is attempted in the Appendix B of the present article.

\section{Scope and Outline}

It is not the scope of the present article to review all types of concepts mereotopology beyond of what has shortly been summarized in the introduction. Mereotopology, Mereogeometry and Region Connect Calculus all are based on logical expressions having

\footnotetext{
4 https://en.wikipedia.org/wiki/Semiotic_theory_of_Charles_Sanders_Peirce
} 
only the logical values "true" or "false". The phase-field concept - in contrast - allows for a quantitative, continuous description especially of transitions between different regions. Following George Boolos: "to be is to be the value of a variable or some values of some variables" [46], the value of the phase-field variable identifies anything as being a fraction of the universe or of a region under consideration. Any phase-field variable accordingly takes values from the closed interval $[0,1]$ of the rationale numbers ${ }^{5}$.

The article starts from a short introduction into time-independent phase-field models, which represent objects/regions as scalar fields, the so called phase-fields. It will be shown how boundaries can be represented as correlations of such scalar fields. Along with this basic introduction, analogies and correspondences to mereotopology will already be indicated wherever possible and meaningful. A special section will discuss the extension of the description of dual-boundaries towards higher order junctions like triple junctions and quadruple junctions in which more than two things collocate and coexist.

A dedicated chapter - in a summarizing way - then compares expressions derived from the phase-field concept with their counterparts in the Region Connect Calculus and in classical mereotopology, respectively. It is however beyond the scope of the present article to discuss implications of the phase-field perspective for all types of more complex MT theories.

Current applications of the multiphase-field concept especially address the evolution of complex structures in space and time. A dedicated section of the present article thus introduces the time perspective of the phase-field approach leading to extended notions of isConnected like isTimeConnected ("coexistence"), isSpaceConnected ("collocation"), isPhysicallyConnected, and isCausallyConnected. Further notions becoming possible on the basis of the phase-field concept like wasPhysicallyConnected; isPathConnected or isEnergeticallyConnected are shortly introduced and provide a promising outlook on possible future developments of mereotopology towards "mereophysics".

\section{Phase-Field Models}

Not a single thing can be thought without a contrast to at least one other thing. Any thing thus has at least one "neighbor thing". They form a boundary. They are connected. Multiple things form multiple dual boundaries, but further also lead to the formation of triple and quadruple boundary regions, where multiple things coexist and collocate. A method succesfully being applied to decribe objects, their shapes and their boundaries are phase-field models being developed since the end of the last millenium.

\subsection{Short History of Phase-Field models}

Phase-field models in the recent decades have gained tremendous importance in the area of describing the evolution of complex structures e.g. evolving during solidification of technical alloy systems [47,48] and their processing [49]. They belong to the class of theories of phase-transitions, which go back to van der Waals [50], Ginzburg-Landau [51], Cahn \& Hillard [52], Allen \& Cahn [53], and Kosterlitz-Thouless [54]. A phase-field concept was first proposed in a personal note [55] and later published by different authors [56], [57]. The first numerical implementation of a phase field model describing the evolution of complex shaped $3 \mathrm{D}$ dendritic structures [58] attracted the attention of the materials science community. The concept was then further widened towards treating also multi-phase systems [59] and towards coupling to thermodynamic data [60]. Now-

\footnotetext{
5 in a strict sense "fractions" are all members of the set of rationale numbers. There a priori thus seems to be no need to extend to real numbers.
} 
adays a variety of simulation tools in the area on materials simulation draws on this concept e.g. [61],[62],[63] and renders the evolution of complex structures and patterns - including the dynamics of boundaries and triple junctions - possible, Fig 1. Instructive reviews of phase-field modelling are available e.g. in [64], [65].

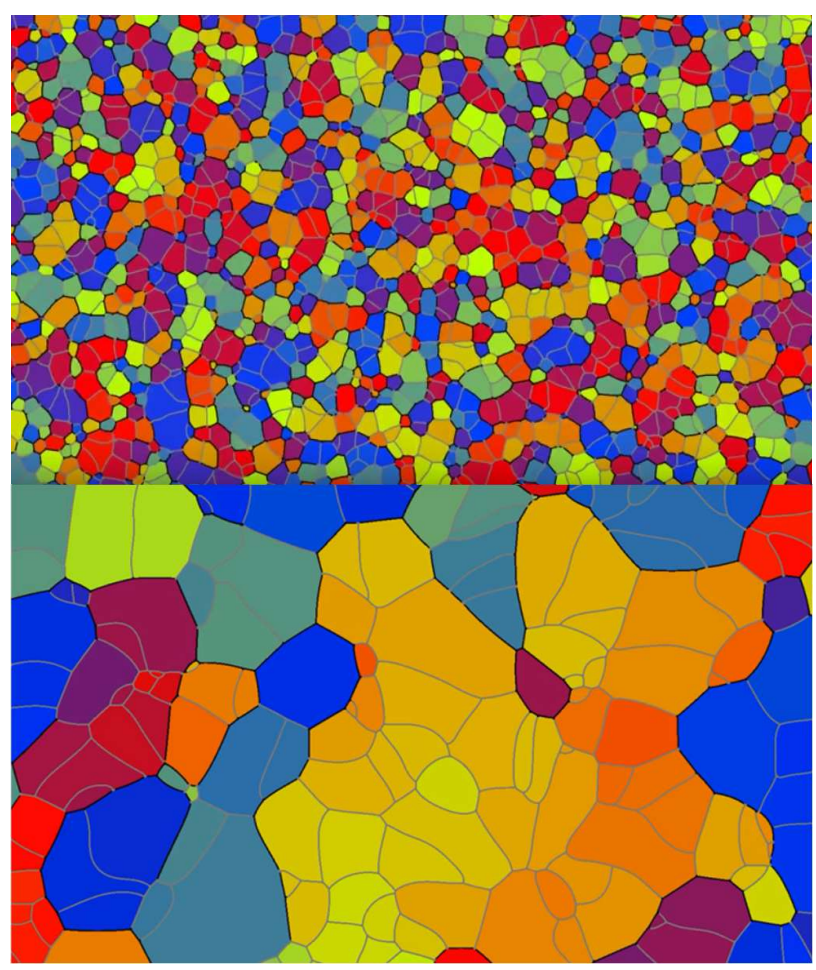

Fig. 1: Grain Growth Process:

Changes in connectivity and in cardinality of the system occur. The initial grain structure (top) evolves towards the grain structure at a later stage (bottom). This grain growth process has been simulated using [61] and a full video of this simulation is available in HD resolution [66].

\subsection{Basic Introduction to Phase-Field Models}

The phase field model in a first place is a way to mathematically describe things and their complex geometrical shape at all, Fig 2.

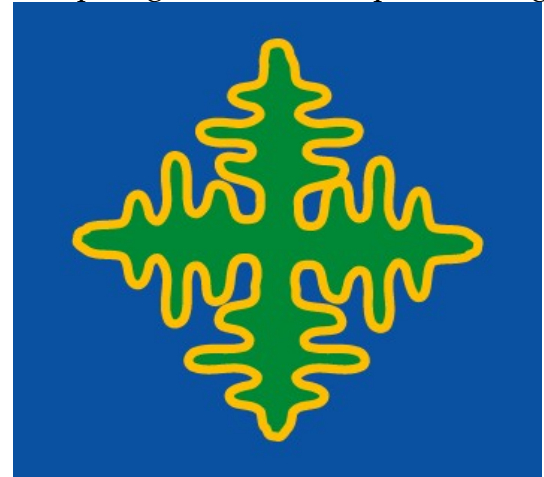

Fig. 2a: A solid phase $\Phi_{\mathrm{s}}$ (green center region) coexisting with a liquid phase $\Phi_{1}$ (blue outer region) in a volume. The fraction solid $\Phi_{\mathrm{s}}$ amounts to approx. $1 / 3$ of the overall volume, while the fraction liquid is approx. $2 / 3$. Both are non-zero and their correlation (yellow) thus exists as a boundary in the overall volume. Nothing can however be said about the position of this boundary without further discretization of the volume (Fig. 2b).

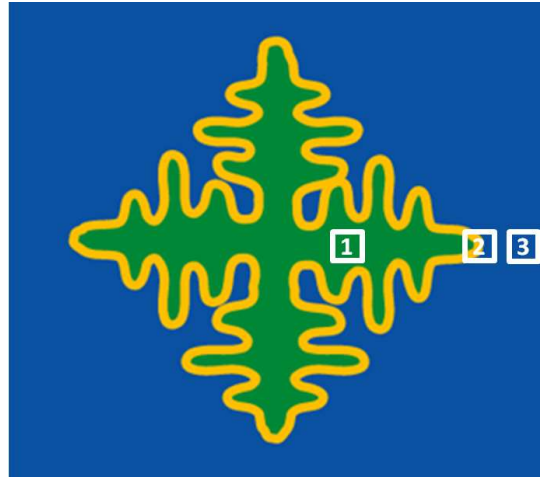

Fig. $2 \mathrm{~b}$ : In above tiny volume " 1 " the fraction solid $\Phi_{\mathrm{s}}$ amounts to exactly 1 , while the fraction liquid $\Phi_{1}$ is exactly 0 . In the tiny volume " 3 " the fraction solid $\Phi_{\mathrm{s}}$ amounts to exactly 0 , while the fraction liquid $\Phi_{1}$ is exactly 1 . In contrast, both fractions are non-zero and their correlation (yellow) exists in the tiny volume " 2 ", which thus comprises a boundary. 
Similar to the Heaviside function $\Theta(x)$ [67], the phase-field function in one dimension $\Phi(\mathrm{x})$ is a function describing the presence or the absence of an object. In contrast to the Heaviside function the phase field function, however, reveals a continuous transition over a finite - though very small - interface thickness $\eta$, Figure3.

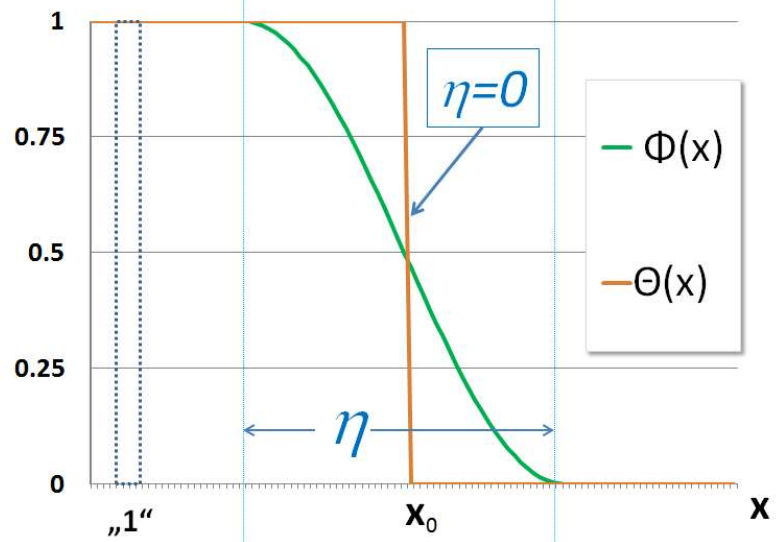

Figure 3: Schematic view of the phase-field function $\Phi(\mathrm{x})$. This function takes a non-zero value wherever the object is present, it takes exactly the value 1 where it is the only present object and is 0 elsewhere (i.e. where the object is absent). It exhibits a continuous transition between two regions over a finite interface thickness $\eta$. The Heaviside function $\Theta\left(\mathrm{x}_{0}\right)$ being characterized by a mathematically sharp transition at $\mathrm{x}_{0}$ is shown as a reference. The dotted region " 1 " exemplarily corresponds to tiny volume " 1 " in Fig 2b) where only the solid is present.

Nothing is a priori known about the exact "shape" of the phase field function in the transition region. Reasoning towards a specification of this shape is based on statistical distributions of gradients in the interface and is described in [68] and [69]. In spite of not knowing this exact shape, a number of terms/expressions can already be qualitatively identified, Table 1, which all allow the identification and description of the transition region (expressions (5), (6), (7 :"overlap"), (8), (13) and (14) in table 1), Figure 4.

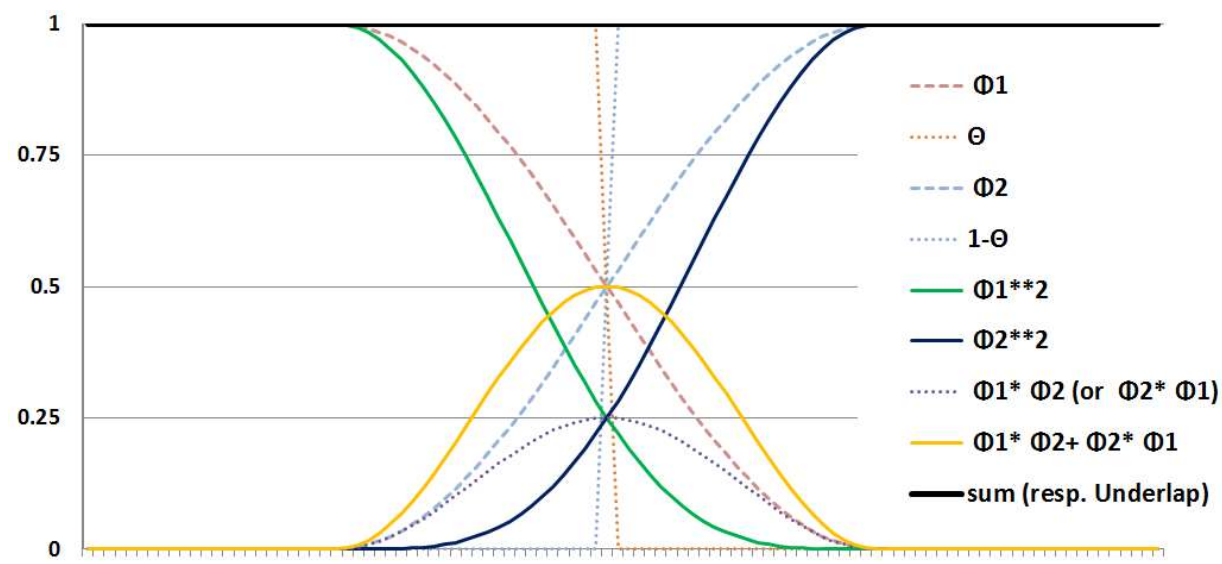

Figure 4: The solid lines represent the three terms occurring in the square of the basic equation (expression 12 in Table 1).

An important characteristic of the phase-field description is that the terms (in expressions (4) and (12) in Table 1) corresponding to the "underlap" in mereology (expression (3)) sum up to a value of 1 everywhere and any time. Expressed in words expression (4) reads:

The whole is the sum of its fractions 
- anywhere, at any time and in any subsystem.

Expressed as a formula this reads:

$1=\sum_{i=0}^{N_{\Phi}} \Phi_{i}(x, t)$ for all $x$ and all $t$ (basic equation)

with $\mathrm{N}_{\Phi}$ being the number of things and $\Phi_{0}$ being the matrix/background thing [1].

In detail this equation - which has a very strong relation to the "mereological sum" defined in mereotopology (see section 4) - means (i) that at least one thing is always present - i.e. has a non- zero value, (ii) that if a thing is the only - single - thing it takes the value of exactly 1 (and all others take exactly the value 0 ), (iii) that if multiple things exist (i.e. have non-zero values), none of them reaches the value of 1 , (iv) that if multiple things exist also their correlations exist (expression 12) and (v) that if multiple things exist (i.e. have non zero values) the sum of all their values equals to 1 . Any transformation, any evolution, any mereotopological description is subject to this "normalization" constraint. 


\begin{tabular}{|c|c|c|c|c|c|}
\hline \multicolumn{2}{|c|}{$\begin{array}{l}\text { Table 1: Quantification of boundaries } \\
\text { in simple phase-field models. } \\
\text { Expressions (3) and (7) indicate } \\
\text { links/correspondances to mereology. }\end{array}$} & $\uparrow$ & ?? & $\eta \quad r$ & \multirow[b]{2}{*}{ remarks } \\
\hline $\begin{array}{l}\text { Expression/ } \\
\text { Term ID }\end{array}$ & $\begin{array}{l}\text { variable/ } \\
\text { term }\end{array}$ & $\begin{array}{l}\text { Value in } \\
\text { bulk } 1\end{array}$ & $\begin{array}{l}\text { Value in } \\
\text { boundary }\end{array}$ & $\begin{array}{c}\text { Value in } \\
\text { bulk } 0\end{array}$ & \\
\hline (1) & $\Phi_{1}$ & 1 & $0<\Phi_{1}<1$ & 0 & \\
\hline (2) & $\Phi_{0}$ & 0 & $0<\Phi_{0}<1$ & 1 & \\
\hline (3) & $\Phi_{0} \vee \Phi_{1}$ & $\begin{array}{c}1 \\
\text { (true) }\end{array}$ & $\begin{array}{c}\text { I } \\
\text { (true) }\end{array}$ & $\begin{array}{c}1 \\
\text { (true) }\end{array}$ & $\begin{array}{l}\text { corresponds to } \\
\text { "mereological } \\
\text { sum" (section 4) }\end{array}$ \\
\hline (4) & $\sum \Phi_{i}$ & 1 & 1 & 1 & $\begin{array}{l}\text { basic equation } \\
\text { (see [1]) }\end{array}$ \\
\hline (5) & $\Phi_{0} \Phi_{1}$ & 0 & $0<\Phi_{0} \Phi_{1}<1$ & 0 & $\begin{array}{l}\text { pairwise } \\
\text { correlation }\end{array}$ \\
\hline (6) & $\Phi_{1} \Phi_{0}$ & 0 & $0<\Phi_{1} \Phi_{0}<1$ & 0 & $\begin{array}{l}\text { same as (5) but } \\
\text { not necessarily } \\
\text { commutative }\end{array}$ \\
\hline$(5 a) /(6 a)$ & & 0 & $\begin{array}{l}0<\partial_{0,1}<1 \\
0<\partial_{1,0}<1\end{array}$ & 0 & $\begin{array}{l}\text { introduction of } \\
\text { nomenclature for } \\
\text { a dual boundary } \\
\text { (see text) }\end{array}$ \\
\hline (7) & $\Phi_{0} \wedge \Phi_{1}$ & $\begin{array}{c}0 \\
\text { (false) }\end{array}$ & $\begin{array}{c}\text { I } \\
\text { (true) }\end{array}$ & $\begin{array}{c}0 \\
\text { (false) }\end{array}$ & $\begin{array}{l}\text { corresponds to } \\
\text { "overlap" in } \\
\text { mereology }\end{array}$ \\
\hline (8) & $\sum \Phi_{i} \Phi_{j}$ & 0 & $0<\sum \Phi_{i} \Phi_{j}<1$ & 0 & $\begin{array}{l}\text { sum of all } \\
\text { pairwise } \\
\text { correlations }\end{array}$ \\
\hline (9) & $\Phi_{1}^{2}$ & 1 & $0<\Phi_{1}^{2}<1$ & 0 & \\
\hline (10) & $\Phi_{0}^{2}$ & 0 & $0<\Phi_{0}^{2}<1$ & 1 & \\
\hline (11) & $\sum \Phi_{i}^{2}$ & 1 & $0<\sum \Phi_{i}^{2}<1$ & 1 & \\
\hline (12) & $\left(\sum \Phi_{i}\right)^{2}$ & 1 & 1 & 1 & $\begin{array}{l}\text { square of basic } \\
\text { equation; see [1] }\end{array}$ \\
\hline (13) & $\left(\sum \Phi_{i}\right)^{2}-\sum \Phi_{i}^{2}$ & 0 & $\sum \Phi_{i} \Phi_{j}$ & 0 & $\begin{array}{l}\text { see [1] and the } \\
\text { present article }\end{array}$ \\
\hline (14) & $\sum \Phi_{i}-\sum \Phi_{i}^{2}$ & 0 & $-\sum \Phi_{i} \ln \Phi_{i}$ & 0 & $\begin{array}{l}\text { for reasoning } \\
\text { towards this } \\
\text { entropy type } \\
\text { formulation } \\
\text { see [1] }\end{array}$ \\
\hline
\end{tabular}

To describe geometric structures, the phase-field function can be considered as being a function of space only. In this case the function does not depend on time and spatial structures are considered to be eternal and thus not to change. "Collocation" then is the key to describe boundaries and the relation isConnected becomes a synonym for isCollocated. "Collocation" in a first place requires the two things $\Phi_{\mathrm{k}}$ and $\Phi_{\mathrm{n}}$ to exist individually 
at some - also existing - places $\mathrm{xn}_{\mathrm{n}} \mathrm{xl}$, which are tiny, but finite volumes inside the region of interest:

$$
\begin{gathered}
\Phi_{i} \text { exists at } x_{n} \equiv \exists x_{n} \wedge \Phi_{i}\left(x_{n}\right) \neq 0 \\
\Phi_{k} \text { exists at } x_{l} \equiv \exists x_{l} \wedge \Phi_{k}\left(x_{l}\right) \neq 0 \\
\Phi_{i} \text { isCollocated } \Phi_{k} \equiv \exists x_{0} \text { such that } \Phi_{i}\left(x_{0}\right) \neq 0 \wedge \Phi_{k}\left(x_{0}\right) \neq 0
\end{gathered}
$$

This expression describes the collocation of two things in a tiny volume $\mathrm{x}_{0}$. It is equivalent to a non-vanishing algebraic product describing the spatial correlation $\Phi_{\mathrm{i}} \Phi_{\mathrm{k}}$ in that tiny volume (see Table 1 ):

$$
\Phi_{i} \text { isCollocated } \Phi_{k} \rightarrow \Phi_{i}\left(x_{0}\right) \Phi_{k}\left(x_{0}\right) \neq 0
$$

The boundary between these two things thus can be defined as the set of all those volume elements $x_{n}$ in which this correlation does not vanish. Summing up all these non-vanishing spatial correlations over all $\mathrm{N}_{\mathrm{x}}$ tiny volumes $\mathrm{x}_{\mathrm{n}}$ constituting the overall volume of the system under consideration yields the fraction, which the dual boundary between $\Phi_{\mathrm{i}}$ and $\Phi_{\mathrm{k}}$ takes of that total volume:

$$
\partial \Phi_{i, k}=\frac{1}{N_{x}} \sum_{n=1}^{N_{x}} \Phi_{i}\left(x_{n}\right) \Phi_{k}\left(x_{n}\right)
$$

The symbol " $\partial$ " has been introduced here to denote a boundary. This symbol is typically used in mathematics to denote the boundary $\partial \Omega$ of a region $\Omega$ (see also introduction of this notation in table 1 ). The boundary between two things $\mathrm{i}, \mathrm{k}-$ which is a volume - is related to the sum of all volume elements $\mathrm{x}_{\mathrm{n}}$ where correlations between thing $i$ and thing $\mathrm{k}$ are non-vanishing. Any of the things may have further boundaries also with other things. The total boundary of thing $\Phi_{i}$ then - in lowest order of all its dual boundaries - is given by the sum of its dual boundaries with all $(\forall)$ other things:

$$
\partial \Phi_{i, \forall}=\frac{1}{N_{x}} \sum_{\substack{i=0 \\ i \neq k}}^{N_{\Phi}} \sum_{j=1}^{N_{x}} \Phi_{i}\left(x_{j}\right) \Phi_{k}\left(x_{j}\right)
$$

This is the same as

$$
\partial \Phi_{i, \forall}=\sum_{\substack{k=0 \\ k \neq i}}^{N_{\Phi}} \partial \Phi_{i, k}
$$

$\partial \Phi_{i, k}$ being identical to 0 means that no interface at all exists between thing $\mathrm{i}$ and thing $\mathrm{k}$ : neither in the considered domain, nor in any of its sub-domains, nor in any of its elementary volume elements.

\subsection{Multi-Phase-Field Models}

Classical phase field models - and most other theories of phase-transitions and also most mereotopological approaches - describe the boundary or connection between exactly two things (resp. the transition between exactly two states). In many areas of applications, however, situations occur, where three or more things coexist and collocate. An instructive practical example is the so called peritectic reaction occurring during solidification of a steel grade, Fig. 5: 


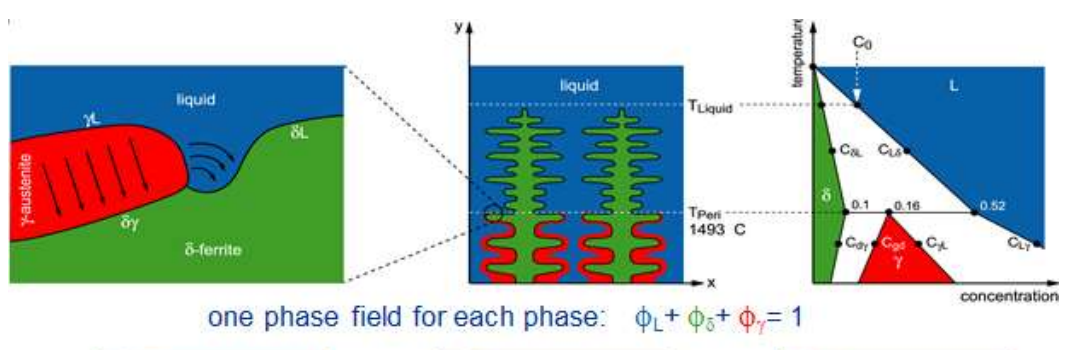

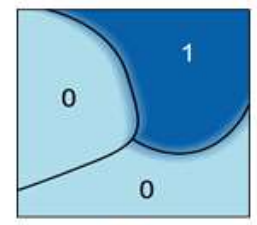

$\phi_{\mathrm{L}}$

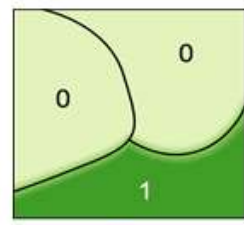

$\phi_{\delta}$

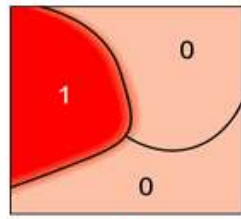

$\phi_{\gamma}$

Fig. 5: Schematic of a peritectic reaction in a steel grade. Red/green/blue areas indicate regions being occupied by the three phases: austenite $(\gamma)$ ferrite $(\delta)$ and liquid melt (L). Wherever there is only (!) Liquid, no ferrite and no austenite may be present. The phases pairwise coexist and collocate at their dual boundaries. All three phases coexist and collocate at the triple junction in the middle of each picture. The upper right inset (the phase-diagram) indicates that the phases also coexist in the same energy interval (i.e. at the same temperature). Note the "Basic Equation" for the three things in the center of the figure.

To account for such configurations the multi-phase-field concept has been developed [59], which allows for the description of structures comprising multiple objects/things like different phases as depicted in Fig 5. or for multiple grains of a single phase as depicted in Fig. 1. In this multi-phase-field model the basic equation enters as a constraint into the Lagrange density forming the basis for the derivation of the evolution equations for the different phase-fields.

\subsection{Triple junctions}

Higher order junction terms correspond to correlations of three (for triple junctions) or even more things (further, higher order junction terms). In multi-phase-field models triple junction terms are necessary to describe the equilibrium wetting angles like e.g. formed by a droplet on a solid substrate placed in air satisfying "Young's Law" [70] and especially the kinetics of motion of such triple junctions. A detailed analysis of triple junctions and their role in phase-field models in microstructure evolution is given in [71].

As triple junctions are relevant for the description and discussion especially of "contacts" in the RCC and in mereotopology in section 4, they are explicitly formulated here. For this purpose the basic equation (expression (4) in table 1) has to be formulated for at least three things and has to be - at least - cubed. Simple squaring of the basic equation even for three things will not generate triple junction terms.

$$
\begin{gathered}
1=\left(\Phi_{i}+\Phi_{j}+\Phi_{k}\right)^{3}=\Phi_{i}^{3}+\Phi_{i}^{2} \Phi_{k}+\Phi_{i}^{2} \Phi_{j}+\Phi_{i} \Phi_{k}^{2}+\Phi_{i} \Phi_{j}^{2}+\Phi_{i} \Phi_{j} \Phi_{k}+\Phi_{i} \Phi_{k} \Phi_{j} \\
(+\cdots+\cdots+\cdots \text { permutations of } i, j, k)
\end{gathered}
$$

The volume term (first term of the RHS) for the bulk fraction, where only the one object $i$ exists then reads 


$$
\partial \Phi_{i}=\frac{1}{N_{x}} \sum_{n=1}^{N_{x}} \Phi_{i}^{3}\left(x_{n}\right)
$$

The dual boundary fractions ( $2^{\text {nd }}$ and $4^{\text {th }}$ term for $i, k$, resp. $3^{\text {rd }}$ and $5^{\text {th }}$ term for $i, j$ ) for the region $\mathrm{i}$ with the other region $\mathrm{k}$ in this ternary case then read:

$$
\partial \Phi_{i, k}=\frac{1}{N_{x}} \sum_{n=1}^{N_{x}} \Phi_{i}\left(x_{n}\right) \Phi_{k}^{2}\left(x_{n}\right)+\frac{1}{N_{x}} \sum_{n=1}^{N_{x}} \Phi_{i}^{2}\left(x_{n}\right) \Phi_{k}\left(x_{n}\right)
$$

This expression reduces to the binary case if $\Phi_{j}=0$ :

$$
\begin{gathered}
\partial \Phi_{i, k}=\sum_{n=1}^{N_{x}} \Phi_{i}\left(x_{n}\right) \Phi_{k}\left(x_{n}\right) \Phi_{k}\left(x_{n}\right)+\sum_{n=1}^{N_{x}} \Phi_{i}\left(x_{n}\right) \Phi_{i}\left(x_{n}\right) \Phi_{k}\left(x_{n}\right) \\
\partial \Phi_{i, k}=\sum_{n=1}^{N_{x}} \Phi_{i}\left(x_{n}\right) \Phi_{k}\left(x_{n}\right) \Phi_{k}\left(x_{n}\right)+\Phi_{i}\left(x_{n}\right) \Phi_{i}\left(x_{n}\right) \Phi_{k}\left(x_{n}\right) \\
\partial \Phi_{i, k}=\sum_{n=1}^{N_{x}} \Phi_{i}\left(x_{n}\right) \Phi_{k}\left(x_{n}\right)\left[\Phi_{k}\left(x_{n}\right)+\Phi_{i}\left(x_{n}\right)\right]
\end{gathered}
$$

with the sum in the square brackets being equal to 1 if $\Phi \mathrm{j}=0$ :

$$
\partial \Phi_{i, k}=\sum_{n=1}^{N_{x}} \Phi_{i}\left(x_{n}\right) \Phi_{k}\left(x_{n}\right)
$$

The two ternary terms - the triple junction terms 6 and 7 on the RHS of the equation - contributing to the boundary of region i read:

and

$$
\partial \Phi_{i, j, k}=\sum_{n=1}^{N_{x}} \Phi_{i}\left(x_{n}\right) \Phi_{j}\left(x_{n}\right) \Phi_{k}\left(x_{n}\right)
$$

$$
\partial \Phi_{i, k, j}=\sum_{n=1}^{N_{x}} \Phi_{i}\left(x_{n}\right) \Phi_{k}\left(x_{n}\right) \Phi_{j}\left(x_{n}\right)
$$

These two terms -being permuted in the last two indices- correspond to two different types of triple junctions revealing a different helicity. The topic helicity is discussed in a separate section below. The total boundary of a thing $i$ then is the sum of its dual boundaries plus the triple junction terms:

$$
\partial \Phi_{i, \forall}=\sum_{\substack{k=0 \\ k \neq i}}^{3} \partial \Phi_{i, k}+\sum_{\substack{j, k=0 \\ k \neq i \neq j}}^{3} \partial \Phi_{i, j, k}
$$

Based on the these specifications of boundary and triple junction terms, mereotopological relations can be easily be formulated and visualized based on some simple configurations as will be shown in section 4 . It is important to note that all current mereotopological relations between two things will probably profit from the introduction/notion of a third thing, the "background" or "matrix" thing 0 , to which they are connected as well, Fig. 6. 


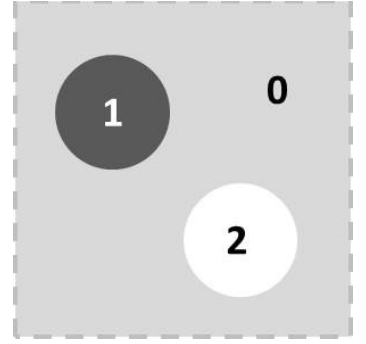

No triple junction:

1 isDisconnected 2

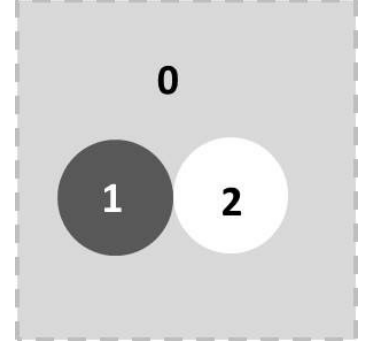

One triple junction:

1 isExternalContact 2

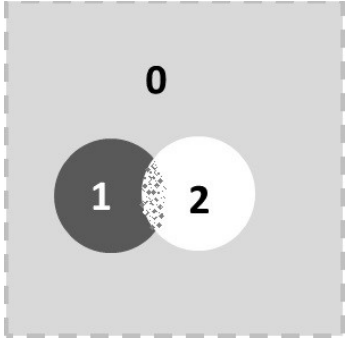

Two triple junctions: 1 isPartOf 2

Figure 6: Classification of different configurations known from mereotopology and region connect calculus according to the number of triple junctions being present in the system. Dyadic relations between two things turn into triadic relations of three things if the matrix thing $(0)$ is taken into account.

The simple examples depicted in Fig. 6 are introduced here to highlight the role of the "background" resp. "matrix" thing " 0 " and also the importance of triple and higher order junctions for mereotopology in general. The class "no triple junction" besides the case "isDisconnected" also contains the case "isNonTangentialProperPart", the class "one triple junction" applies to "isExternalContact" and also to "isTangentialProperPart". Eventually the presence of two triple junctions corresponds to the "isPartOf" relation.

Additional remark: The three configurations of things depicted in Fig. 6 might also be considered as a temporal sequence of two individuals ("atoms") being initially disconnected and then entering into a bound state. This scenario relates to Mulliken's holistic interpretation of a bound state [72], where the two atomic orbitals must incorporate "the overlap in the space region that corresponds to the intersection of each atomic space" [73]. A mereology of quantum chemical systems has recently been discussed [74].
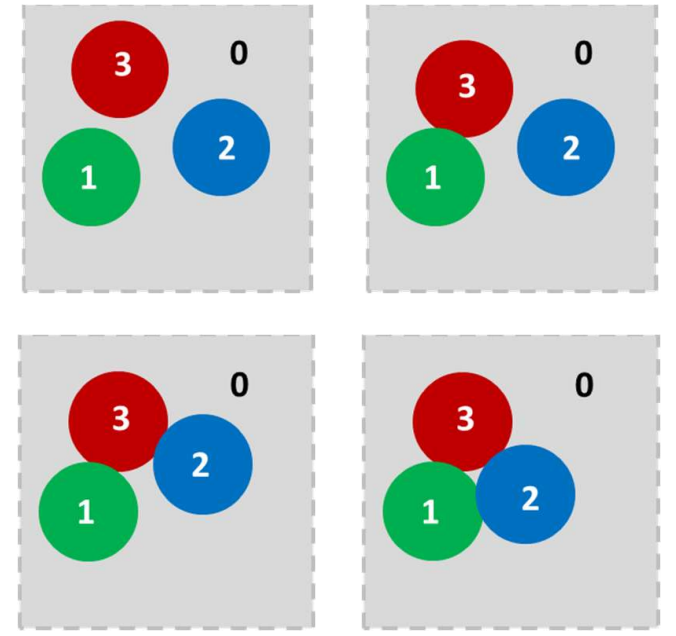

Fig.7 a: Multiple things in a volume. The two situations left can be described well by classical mereotopology. Most left: All things are mutually disconnected resp connected with the matrix only. Left: 1 isPartOf 3 ,

2 isDisconnected from both 1 and 3 differ in number and type of triple junctions. This number increases from 4 (left) to 6 (middle) all of which include the matrix thing. Eventually (right) a situation of 4 triple junctions is possible in which one of these triple junctions does not comprise the matrix thing. Note that the number of triple junctions is always even. The case of "external contact" seemingly having only one triple junction is discussed separately (see text).

In case all three things being connected to each of the other things (see Figure 7 lower right and Figure 8) there are three dual boundaries. Starting from the bottom and crossing these boundaries counter-clockwise, Figure 8 (left) gives following sequence: red-blue , blue-green, green-red, which we abreviate for the ease of further reading as 
R-B, B-G, G-R or even simpler (omitting also the hyphons) as RB, BG, GR. Thus "RB" reprents a transition from red to blue. This is definitely something to be distinguished from a transition "BR" going from blue to red. $\mathrm{RB}$ thus is not the same as BR. The sequence of the symbols being used to denote the boundary thus is important and has a meaning. It denotes the direction in which the boundary is crossed. Taking the convention of reading letters from left to right (as usual in most western languages) one will start from red to blue. In contrast, when taking the convention of reading signs from right to left (as e.g. in Arabian language) the red to green transition would be the first. Even more interesting is to have a look at the sequence when going from one area to the next neighboring area. Again starting on the bottom (the red region in Fig.8) the sequence reads R-B-G (and eventually back to red: $-\mathrm{R}$ ). But one could also start from blue and continue in so- called cyclic permutations :

$$
\mathrm{R}-\mathrm{B}-\mathrm{G} \text { is the same as } \mathrm{B}-\mathrm{G}-\mathrm{R} \text { is the same as } \mathrm{G}-\mathrm{R}-\mathrm{B}
$$

One will however never end (for this triple symbol AND when continuing going clockwise! ) in a situation:

\section{$\mathrm{R}-\mathrm{G}-\mathrm{B}$ is the same as G-B-R is the same as B-R-G}

The sequence of letters used to describe these two symbols - in 2 dimensions - thus either allows (i) distinguishing two different types of triple junctions or (ii) describing a sense of rotation (clockwise/counterclockwise), Fig. 8 (left) and Fig. 8 (right).
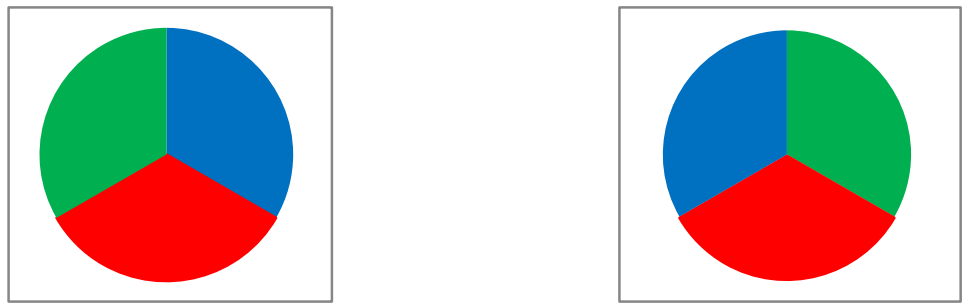

Figure 8 : Two different triple juntions in 2 dimensions in which three objects red (R), green $(\mathrm{G})$, and blue $(\mathrm{B})$ coexist and collocate. They can be distinguished by the sequence in which the three things are arranged. They cannot be mapped onto each other by any rotation in 2D (see text), but only by mirroring. They may be - and in 3D physical systems probably are - connected in the third dimension (see text and Fig.

10) meaning that they are parts of the same thing.

Triple junctions are regions of collocation of three things. They are also regions where the three dual boundary planes (which each are volumes being thin in one direction) between the three differnet things collocate. As boundaries between any pair of two things in 3D are "planes", the intersection of any pair of "planes" defines a "line" In three dimensions. Triple junctions thus are "lines" (having finite volumes), which in 3 dimensions either form closed loops - called vortices - or are connected to the boundary of the region of interest, respectively. Triple junctions are not "points" as they seem to be in a 2 D section, but lines. In 2D sections they always appear as "pairs", Figure 9. 


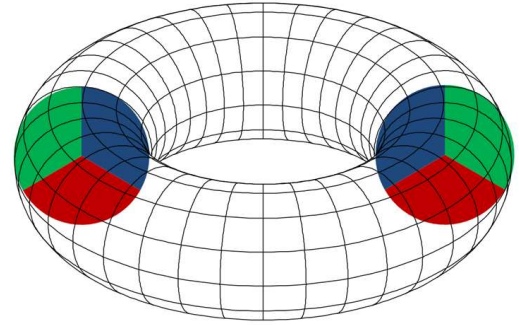

Fig.9a) Visualisation of a vortex resp. of a torus. A 2D section reveals two vortcies with opposite helicities.

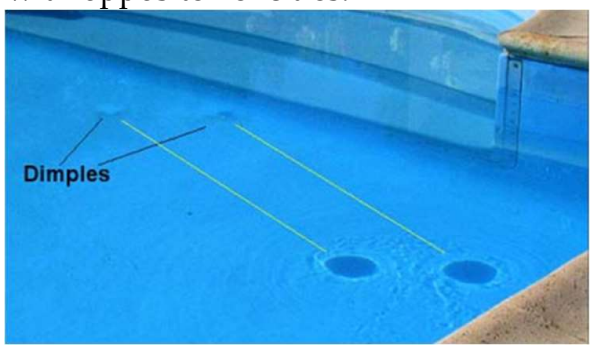

Fig.9c) Triple junctions in a 2 D section at the water surface always show up as pairs, shown here for two flow vortices ("dimples") in a pool - so called Falaco solitons [76]. "Falaco solitons appear to have properties claimed by the String theorists trying to explain Quantum Gravity" [77].

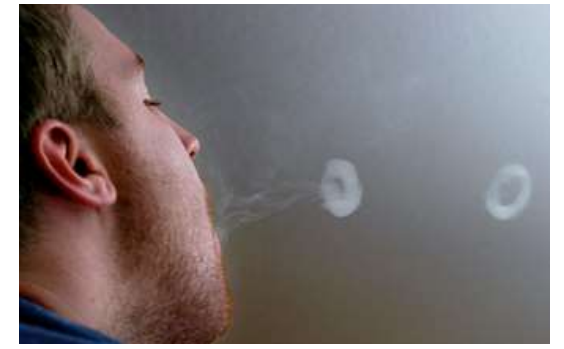

Fig.9b) Smoke vortices as propagaing objects in a 3D world. Photo reproduced from [75]

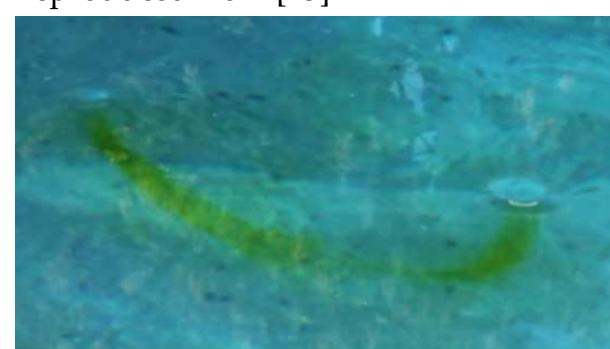

Fig. 9d) Experimental visualisation of 3-D conectivity of two vortices [78]. The surface dimples have been couloured with green fluid, which reveals them being coupled in the third dimension in a semi vortex extending under the water surface.

\subsection{Quadruple and higher order junctions}

A maximum of 4 things can coexist at the same position "i.e. coexist and collocate". In such a case they form quadruple "points". Such quadruple points only exist in 3D space. Remember that all "points", "lines" and "planes" are 3D objects (in a 3D world) resp. 4D objects in a $4 \mathrm{D}$ world (see Appendix A). There are

- pairs of things forming dual boundaries ( being "planes")

- 3 coexisting/collocated things forming 3 dual boundaries being coexisting/ collocated in a triple boundary (being a "line")

○ 4 coexisting things have following boundaries as parts 1 quadruple "point", 4 triple "line" junctions, and 6 dual boundary "planes"

The fourth power of the basic equiation for 4 things yields total of $4^{4}=256$ terms and can be sorted using the multinomial expansion ${ }^{6}$ :

$$
\left(\Phi_{1}+\Phi_{2}+\Phi_{3}+\Phi_{4}\right)^{4}=\sum_{k 1+k 2+k 3+k 4=4} \frac{4 !}{k 1 ! k 2 ! k 3 ! k 4 !} \Phi_{1}^{k 1} \Phi_{2}^{k 2} \Phi_{3}^{k 3} \Phi_{4}^{k 4}
$$

The $\mathrm{k}_{\mathrm{i}}$ always sum up to 4 by definition of the sum. This is further detailed in Appendix $\mathrm{C}$ and eventually allows classifying into

- 4 "unary" terms $\partial \Phi_{i}$ with one ot the ki equals 4 and the others are identical 0

\footnotetext{
6 see e.g https://en.wikipedia.org/wiki/Multinomial_distribution
} 
- 84 "dual" boundary terms, where two of the ki are identical 0 and the others complement to 4 . For a dual boundary $i, j$ this provides 14 terms: $\partial \Phi_{i, j}$ (7terms) and $\partial \Phi_{j, i}(7$ terms). A total of 6 boundary pairs (i,j; i,k; i,l; k,l; $\mathrm{j}, \mathrm{k}$ and $\mathrm{j}, \mathrm{l})$ thus generates the total of 84 dual boundary terms.

- 144 "triple" boundary terms, where one of the $\mathrm{k}_{\mathrm{i}}$ is identical 0 and the three others complement to 4 . Each triple junction $\mathrm{i}, \mathrm{j}, \mathrm{k}$ generates 36 terms, which can be classified according to the helicity of the junction:

$$
\partial^{+} \Phi_{i, j, k}(18 \text { terms })+\partial^{-} \Phi_{i, j, k}(18 \text { terms })=\partial \Phi_{i, j, k}(36 \text { terms })
$$

A total of 4 triple sets $(i, j, k ; i, j, l ; i, k, l$ and $j, k, l)$ thus generates the total of 144 triple boundary terms.

- 24 "quadruple" boundary terms, where all $\mathrm{k}_{\mathrm{i}}$ are identical 1 leading to (sorted by first index)

$$
\partial \Phi_{i, j, k, l}\left(6 \text { terms); } \partial \Phi_{j, i, k, l}(6 t e r m s) ; \partial \Phi_{k, i, j, l}(6 t e r m s) ; \partial \Phi_{l, i, j, k}(6 t e r m s)\right.
$$

In summary the following overall equation scheme results:

$$
\begin{gathered}
\Phi_{i}=\partial \Phi_{i}+\sum_{\substack{j \\
i \neq j}} \partial \Phi_{i, j}+\sum_{\substack{j, k \\
i \neq j \neq k}}\left[\partial^{+} \Phi_{i, j, k}+\partial^{-} \Phi_{i, j, k}\right]+\sum_{\substack{j, k, l \\
i \neq j \neq k \neq l}} \partial \Phi_{i, j, k, l} \\
\Phi_{j}=\partial \Phi_{j}+\sum_{\substack{i \\
j \neq j}} \partial \Phi_{j, i}+\sum_{\substack{i, k \\
i \neq j \neq k}}\left[\partial^{+} \Phi_{j, i, k}+\partial^{-} \Phi_{j, i, k}\right]+\sum_{\substack{i, k, l \\
i \neq j \neq k \neq l}} \partial \Phi_{j, i, k, l} \\
\Phi_{k}=\partial \Phi_{k}+\sum_{\substack{j \\
j \neq k}} \partial \Phi_{k, j}+\sum_{\substack{i, j \\
i \neq j \neq k}}\left[\partial^{+} \Phi_{k, i, j}+\partial^{-} \Phi_{k, j, i}\right]+\sum_{\substack{i, j, l \\
i \neq j \neq k \neq l}} \partial \Phi_{i, j, k, l} \\
\Phi_{l}=\partial \Phi_{l}+\sum_{\substack{j \\
j \neq l}} \partial \Phi_{l, j}+\sum_{\substack{j, k \\
i \neq j \neq k}}\left[\partial^{+} \Phi_{i, j, k}+\partial^{-} \Phi_{i, j, k}\right]+\sum_{\substack{i, j, k \\
i \neq j \neq k \neq l}} \partial \Phi_{l, j, k, i} \\
1=\sum_{i=1}^{4} \partial \Phi_{i}+\sum_{i=1}^{4} \sum_{\substack{j \\
j \neq i}} \partial \Phi_{i, j}+\sum_{\substack { i=1 \\
\begin{subarray}{c}{j \neq k \\
i \neq j \neq k{ i = 1 \\
\begin{subarray} { c } { j \neq k \\
i \neq j \neq k } }\end{subarray}}\left[\partial^{+} \Phi_{i, j, k}+\partial^{-} \Phi_{i, j, k}\right]+\sum_{\substack{i, j, k, l \\
i \neq j \neq k \neq l}} \partial \Phi_{l, j, k, i}
\end{gathered}
$$

This eventually yields the total fractions of the 4 different objects (the LHS) as these are summed up from contributions of bulk, dual, triple and quadruple boundaries. A visual impression of the different terms for bulks, dual boundaries, triple and quadruple junctions is depicted in Fig.10.
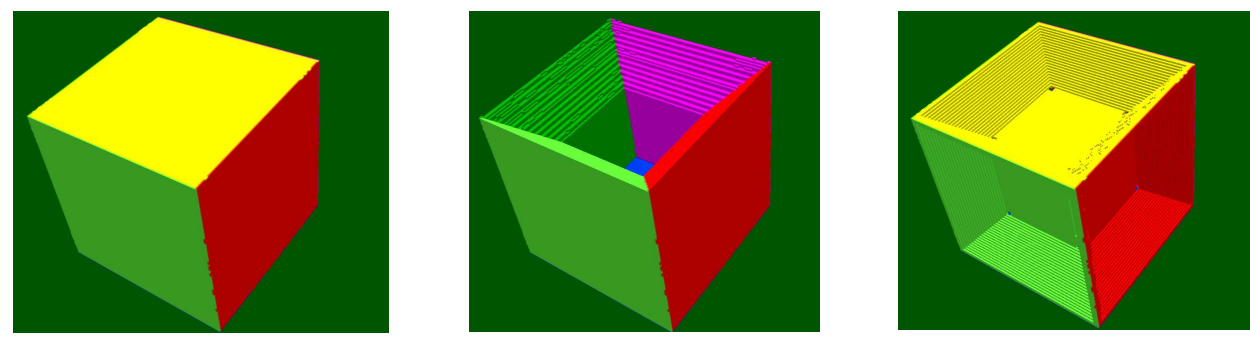

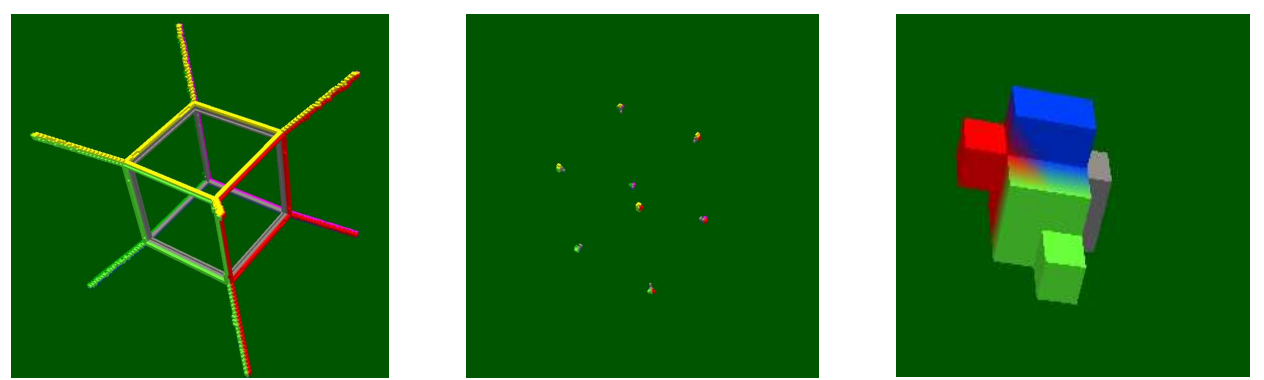

Fig.10: Volumes, Faces/Areas, Egdes/Lines and Vortices/Quadruple Points in a tesseract cube. Upper row: ouside view of the tesseract (left), center cube and one of the faces removed (middle) and dual boundaries (right). Lower row: triple lines (left), quadruple points (middle) and zoom-in into a quadruple point (right). The tesseract structure was synthesized using [61].

\subsection{Summary of phase-field expressions}

In view of the comparison with expressions from Region Connect Calculus, from Contact algebra and from mereology in the following sections, Table 2 provides a list of all terms being necessary for this purpose.

\begin{tabular}{|c|c|c|c|}
\hline $\begin{array}{c}\text { equation } \\
\#\end{array}$ & $\begin{array}{c}\text { global } \\
\text { value }\end{array}$ & $\begin{array}{c}\text { local value in } \\
\text { volume } \mathrm{n}\end{array}$ & relation global-local \\
\hline 1 & $\Phi_{i}$ & $\Phi_{i}\left(x_{n}\right)$ & $\Phi_{i}=\frac{1}{N_{x}} \sum_{n=1}^{N_{x}} \Phi_{i}\left(x_{n}\right)$ \\
\hline 2 & $\partial \Phi_{i}$ & $\partial \Phi_{i}\left(x_{n}\right)$ & $\partial \Phi_{i}=\frac{1}{N_{x}} \sum_{n=1}^{N_{x}} \partial \Phi_{i}\left(x_{n}\right)$ \\
\hline 3 & $\partial \Phi_{i, k}$ & $\partial \Phi_{i, k}\left(x_{n}\right)$ & $\partial \Phi_{i, k}=\frac{1}{N_{x}} \sum_{n=1}^{N_{x}} \partial \Phi_{i, k}\left(x_{n}\right)$ \\
\hline 4 & $\partial \Phi_{i, j, k}$ & $\partial \Phi_{i, j, k}\left(x_{n}\right)$ & $\partial \Phi_{i, j, k}=\frac{1}{N_{x}} \sum_{n=1}^{N_{x}} \partial \Phi_{i, j, k}\left(x_{n}\right)$ \\
\hline 5 & $\partial \Phi_{i, j, k, l}$ & $\partial \Phi_{i, j, k, l}\left(x_{n}\right)$ & $\partial \Phi_{i, j, k, l}=\frac{1}{N_{x}} \sum_{n=1}^{N_{x}} \partial \Phi_{i, j, k, l}\left(x_{n}\right)$ \\
\hline 6 & $\partial \Phi_{i, \forall}$ & $\mathrm{n} / \mathrm{a}$ & $\partial \Phi_{i, \forall}=\sum_{k \neq i} \partial \Phi_{i, k}$ \\
\hline
\end{tabular}

Table 2: List of all terms being necessary to express mereotopological and region connect calculus situations. The quadruple terms seem not yet to be needed to address the mereotopological situations being investigated in the literature by now.

Looking at possible extensions to higher order expressions questions emerge like: Why not raising the basic equation to the $6^{\text {th }}$, to the $8^{\text {th }}$ or even higher powers? Is an even power mandatory? Why not go for more than 4 different objects? For small number of objects (one or two or three) an exponent being equal to the number of objects seems sufficient. If only two objects exist, there will be no triple junction and an exponent of 2 can be considered as sufficient. As a first rule of thumb the exponent thus should correspond to the number of objects. An exponent of 4 then seems sufficient - and necessary - to describe all types of geometric coexistence regions (dual boundaries "planes"; triple junctions "lines" and quadruple junctions "points") even if multiple 
objects are collocated. A power of 6 was used in [66] to refine the desription of the dual interface in a solid-liquid two state system by a refined discretisation.

\section{Comparison with mereotopological concepts}

Based on the specifications of bulk areas, boundaries, triple junctions and quadruple junctions depicted in the preceding section, especially in Table 2, mereotopological relations can easily be formulated and visualized based on some simple configurations as outlined in the following.

\subsection{Comparison with Region Connect Calculus}

Some simple Region Connect Calculus (RCC) situations, Fig.11, are individually discussed based on the description of boundary terms as introduced in section 3. Starting from the easily identifiable configurations (X DC Y, X NTTPi Y, X NTTP Y, and X PO Y), the more complex situations involving only one Triple Junction (X EC Y, X TPP Y, X TPPi $\mathrm{Y}$ ) are discussed. Most attention is paid to the case X EQ Y.

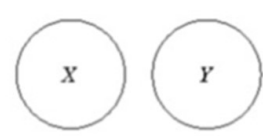

$X$ DC $Y$

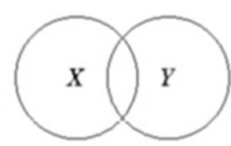

$X$ PO $Y$

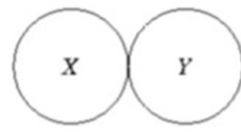

$X$ EC $Y$

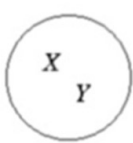

$X$ EQ $Y$

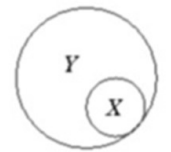

$X$ TPP $Y$

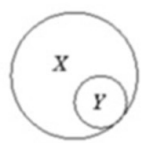

$X$ TPPi $Y$

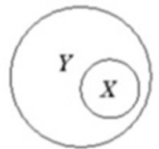

$X$ NTPP $Y$

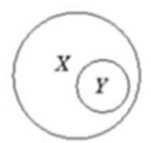

$X$ NTPPi $Y$

Fig. 11 : Region Connection Calculus?: disconnected (DC), externally connected (EC), equal (EQ), partially overlapping (PO), tangential proper part (TPP), tangential proper part inverse (TPPi), non-tangential proper part (NTPP), non-tangential proper part inverse (NTPPi)

Case: X DC Y: X and Y do not have any common boundary:

$$
\partial \Phi_{x, y}=0
$$

For X DC Y, parts X AND Y both have boundaries with the background thing 0 (not shown in the figure) only. Their total boundary thus hasPart only the boundary to the thing 0:

$$
\begin{aligned}
& \partial \Phi_{x, \forall}=\partial \Phi_{x, 0} \\
& \partial \Phi_{y, \forall}=\partial \Phi_{y, 0}
\end{aligned}
$$

As there exists no dual boundary between $X$ and $Y$, also no triple junctions involving either of the two parts do exist:

$$
\begin{array}{ll}
\partial \Phi_{x, y, 0}=0 & \wedge \\
\partial \Phi_{x, 0, y}=0 & \wedge \\
\partial \Phi_{y, 0, x}=0 & \wedge
\end{array}
$$

\footnotetext{
7 https://en.wikipedia.org/wiki/Region_connection_calculus
} 


$$
\partial \Phi_{y, x, 0}=0
$$
addition:

Case: X NTPP Y: X has a boundary with Y only while $Y$ has a boundary to thing 0 in

$$
\begin{gathered}
\partial \Phi_{x, \forall}=\partial \Phi_{x, y} \\
\partial \Phi_{y, \forall}=\partial \Phi_{y, x}+\partial \Phi_{y, 0}
\end{gathered}
$$

Although there exists a boundary between both things, there is no region where both things are connected to the 0 -thing as well. Thus there are no triple junctions:

$$
\begin{aligned}
& \partial \Phi_{x, y, 0}=0 \\
& \partial \Phi_{x, 0, y}=0 \\
& \partial \Phi_{y, 0, x}=0 \\
& \partial \Phi_{y, x, 0}=0
\end{aligned}
$$

Case: X NTPPi Y: Y has a boundary with X only while $X$ has a boundary to thing 0 in addition:

$$
\begin{gathered}
\partial \Phi_{x, \forall}=\partial \Phi_{x, y}+\partial \Phi_{x, 0} \\
\partial \Phi_{y, \forall}=\partial \Phi_{y, x}
\end{gathered}
$$

Though there exists a boundary between both parts, there is no region where both things are connected to the 0 -thing as well. There are no triple junctions:

$$
\begin{aligned}
& \partial \Phi_{x, y, 0}=0 \\
& \partial \Phi_{x, 0, y}=0 \\
& \partial \Phi_{y, 0, x}=0 \\
& \partial \Phi_{y, x, 0}=0
\end{aligned}
$$

All these three cases above do not comprise any triple junction. The easiest case including triple junctions (X PO Y) will be discussed first as this discussion is helpful in understanding the cases revealing one single triple junction only (i.e. the cases $X$ EC $Y, X$ TPP Y, X TPPi Y)

Case: X PO Y: X and Y reveal a region of coexistence/collocation -the overlap region. A boundary between $X$ and $Y$ thus exists:

$$
\partial \Phi_{x, y}>0
$$

Further, both things $\mathrm{X}$ and $\mathrm{Y}$ in this case also have boundaries to thing 0 :

$$
\begin{aligned}
& \partial \Phi_{x, 0}>0 \\
& \partial \Phi_{y, 0}>0
\end{aligned}
$$

X PO Y further comprises two regions ("points") where all three things X AND Y AND the 0-thing collocate: there exist two triple junctions. Their total boundary thus hasPart the boundaries to thing 0 , to $\mathrm{X}$ (resp. to $\mathrm{Y}$ ) and further the two triple junctions where $X, Y$ and 0 coexist/collocate. These two triple junctions however do NOT collocate - i.e. they exist in different volumes $\mathrm{x}_{1}$ and $\mathrm{x}_{\mathrm{m}}$ - and are distinguished by different helicities for the two different locations, see section 3.4. A correlation of the two triple junctions does not exist in any volume element $\mathrm{x}_{\mathrm{n}}$ :

$$
\begin{gathered}
\partial \Phi_{y, \forall}=\partial \Phi_{y, 0}+\partial \Phi_{y, x}+\partial \Phi_{y, x, 0}+\partial \Phi_{y, 0, x} \\
\partial \Phi_{x, \forall}=\partial \Phi_{x, 0}+\partial \Phi_{x, y}+\partial \Phi_{x, y, 0}+\partial \Phi_{x, 0, y} \\
\exists x_{l}: \partial \Phi_{x, y, 0}\left(x_{l}\right) \neq 0 \quad \wedge \quad \exists x_{m}: \partial \Phi_{x, 0, y}\left(x_{m}\right) \neq 0 \quad \wedge \quad \forall x_{n}: \partial \Phi_{x, y, 0}\left(x_{n}\right) \partial \Phi_{x, 0, y}\left(x_{n}\right)=0
\end{gathered}
$$




\section{Case: X EC Y}

In this case there seemingly exists a single triple junction in a discretized single volume $x_{n}$. This defines a "point" (a finite sized smallest volume in 2 dimensions....)

$$
\partial \Phi_{y, 0, x}>0
$$

In the case $X$ PO Y discussed above, two triple junctions are coexistent - but not collocated. In the case X DC Y no triple junctions are existent. X EC Y describes a transition between X DC Y and X PO Y and thus benefits from being capable of describing both cases - each of them as a limit. Looking at the reverse process the two separated - i.e. not collocated - triple junctions in the X POY case then have to condense in to a single state of coexistence AND collocation for the X EC Y case ( and also for the TPP cases..)

$$
\exists x_{n}: \partial \Phi_{x, y, 0}\left(x_{n}\right) \neq 0 \wedge \partial \Phi_{x, 0, y}\left(x_{n}\right) \neq 0 \quad \rightarrow \quad \exists x_{n}: \partial \Phi_{x, y, 0}\left(x_{n}\right) \partial \Phi_{x, 0, y}\left(x_{n}\right) \neq 0
$$

\section{Case: X EQY}

$X$ and $Y$ obviously have the same total boundary:

$$
\partial \Phi_{x, \forall}=\partial \Phi_{y, \forall}
$$

Both have a boundary with 0 only

$$
\begin{aligned}
& \partial \Phi_{x, \forall}=\partial \Phi_{x, 0} \\
& \partial \Phi_{y, \forall}=\partial \Phi_{y, 0}
\end{aligned}
$$

Further, they both have identical phase fields,

$$
\forall x_{n}: \Phi_{x}\left(x_{n}\right)=\Phi_{y}\left(x_{n}\right)
$$

This expression directly implies that the two things are identical i.e. the "same thing" in a mereological sense. They have the same geometry as defined by their individual phase fields. In terms of being fractions of a universe both things are also identical. They can be treated as a single thing representing twice the fraction of one of the two things but losing their identity then:

$$
\forall x_{n}: \Phi_{z}\left(x_{n}\right)=\Phi_{y}\left(x_{n}\right)+\Phi_{x}\left(x_{n}\right)=2 \Phi_{x}\left(x_{n}\right)
$$

Further, there may be additional attributes beyond the mere geometric shape, which may still allow distinguishing them in spite of being geometrically identical.

\subsection{Phase-Field perspective of Contact Algebra}

The topology axioms of contact algebra are (adapted from [18]):

(C1) If $\mathrm{aCb}$, then $\mathrm{a}>0$ and $\mathrm{b}>0$,

(C2) If $\mathrm{aCb}$ and $\mathrm{a} \leq \mathrm{c}$ and $\mathrm{b} \leq \mathrm{d}$, then $\mathrm{cCd}$,

(C3) If $\mathrm{aC}(\mathrm{b}+\mathrm{c})$, then $\mathrm{aCb}$ or $\mathrm{aCc}$,

(C4) If $\mathrm{aCb}$, then $\mathrm{bCa}$,

(C5) If $a \bullet b \neq 0$, then $\mathrm{aCb}$.

In the following these axioms are related and compared to the phase-field formulations for time independent situations. The phase-field formulations introduced in the 
preceding section are shortly recovered in the following for this purpose. A thing $\Phi_{a}$ is globally present in a region under consideration if it takes a non-zero value in this region: $\Phi_{a}>0$

A thing $\Phi_{\mathrm{a}}$ is locally present in a region $\mathrm{x}_{\mathrm{j}}$, which is a sub-region of the region under consideration, if it takes a non-zero value in that sub-region

$$
\Phi_{a}\left(x_{j}\right)>0
$$

The global value $\Phi_{a}$ of is the normalized sum of all local values in the $\mathrm{N}_{\mathrm{x}}$ sub-regions

$$
\Phi_{a}=\frac{1}{N_{x}} \sum_{j=1}^{N_{x}} \Phi_{a}\left(x_{j}\right)
$$

Eventually the definition of "collocation" is:

$$
\Phi_{i} \text { isCollocated } \Phi_{k} \equiv \exists x_{0} \text { such that } \Phi_{i}\left(x_{0}\right) \neq 0 \wedge \Phi_{k}\left(x_{0}\right) \neq 0
$$

which implies that there exists a boundary between the two things

$$
\Phi_{i} \text { is Collocated } \Phi_{k} \rightarrow \partial \Phi_{i, k} \neq 0
$$

"Collocated" in this context (i.e. time independent) means "isSpatiallyConnected" or just is Connected i.e. $\Phi_{i} C \Phi_{k}$ in the nomenclature of contact algebra.

The Axiom (C1) of Contact Algebra -using these phase-field definition of isSpatiallyConnected - then translates into

$$
\begin{gathered}
\Phi_{a} C \Phi_{b} \equiv \partial \Phi_{a, b} \neq 0 \\
\partial \Phi_{a, b} \neq 0 \rightarrow \exists x_{n} \text { such that } \Phi_{a}\left(x_{n}\right) \neq 0 \wedge \Phi_{b}\left(x_{n}\right) \neq 0 \\
\Phi_{a}\left(x_{n}\right) \neq 0 \rightarrow \sum_{j=1}^{N_{x}} \Phi_{a}\left(x_{j}\right) \neq 0 \rightarrow \Phi_{a} \neq 0 \\
\Phi_{b}\left(x_{n}\right) \neq 0 \rightarrow \sum_{j=1}^{N_{x}} \Phi_{b}\left(x_{j}\right) \neq 0 \rightarrow \Phi_{b} \neq 0 \\
\Phi_{a} \subset \Phi_{b} \rightarrow \Phi_{a} \neq 0 \wedge \Phi_{b} \neq 0
\end{gathered}
$$

Expressed in words this reads "If two things are locally connected, both things must at least globally exist". Axiom (C1) thus is recovered by the phase field perspective.

Axiom (C3) covers the case of one thing being connected to two other things, which both globally exist:

$$
\begin{gathered}
\Phi_{a} \neq 0 \wedge \Phi_{b} \neq 0 \wedge \Phi_{c} \neq 0 \\
\Phi_{a} C\left(\Phi_{b}+\Phi_{c}\right) \rightarrow \exists x_{n} \text { such that } \sum_{n=1}^{N_{x}} \Phi_{a}\left(x_{n}\right)\left[\sum_{j=1}^{N_{x}} \Phi_{b}\left(x_{n}\right)+\sum_{k=1}^{N_{x}} \Phi_{c}\left(x_{n}\right)\right] \neq 0 \\
\sum_{n=1}^{N_{x}} \Phi_{a}\left(x_{n}\right) \sum_{j=1}^{N_{x}} \Phi_{b}\left(x_{n}\right)+\sum_{n=1}^{N_{x}} \Phi_{a}\left(x_{n}\right) \sum_{k=1}^{N_{x}} \Phi_{c}\left(x_{n}\right) \neq 0
\end{gathered}
$$




$$
\begin{gathered}
\sum_{n=1}^{N_{x}} \Phi_{a}\left(x_{n}\right) \sum_{j=1}^{N_{x}} \Phi_{b}\left(x_{n}\right) \neq 0 \vee \sum_{n=1}^{N_{x}} \Phi_{a}\left(x_{n}\right) \sum_{k=1}^{N_{x}} \Phi_{b}\left(x_{n}\right) \neq 0 \\
\rightarrow \partial \Phi_{a, b} \neq 0 \vee \partial \Phi_{a, c} \neq 0 \rightarrow \Phi_{a} C \Phi_{b} \vee \Phi_{a} C \Phi_{c}
\end{gathered}
$$

Expressed in words this reads "If a thing is connected to two other things, it is connected to at least one of them". Axiom (C3) thus is recovered also by the phase field perspective. For multiple things this can even be even further refined as: "Any thing is connected to at least one of the other things" or "Any thing is connected to its complement".

Axiom (C4) relates to the symmetry of the connected relation

$$
\begin{gathered}
\Phi_{a} C \Phi_{b} \equiv \exists x_{n} \text { such that } \Phi_{a}\left(x_{n}\right) \neq 0 \wedge \Phi_{b}\left(x_{n}\right) \neq 0 \\
\Phi_{a}\left(x_{n}\right) \neq 0 \wedge \Phi_{b}\left(x_{n}\right) \neq 0 \rightarrow \Phi_{b}\left(x_{n}\right) \neq 0 \wedge \Phi_{a}\left(x_{n}\right) \neq 0 \\
\Phi_{b}\left(x_{n}\right) \neq 0 \wedge \Phi_{a}\left(x_{n}\right) \neq 0 \rightarrow \Phi_{b} C \Phi_{a}
\end{gathered}
$$

Expressed in words this reads: "If thing " $a$ " is connected to thing " $b$ ", then thing " $b$ " is also connected to thing "a"". Axiom (C4) thus is recovered also by the phase field perspective for time - independent correlations.

The situation however may be - and probably is - different for time dependent and other situations. A "path" connecting two things " $a$ " and " $b$ " may exist for some time, while the return path might not exist anymore, when attempting to go back from " $b$ " to "a". An example would be a bridge connecting "a" and "b", which breaks down when crossing it for the first time. The concept of asymmetric connectivity has major implications for a number of areas in physics and chemistry like chemical reactions preferentially proceeding into one direction, entropy always increasing or osmotic processes to name a few. A simple example from everyday life is a one-way in traffic. To the best of the author's knowledge a concept of asymmetric connectivity has not yet been discussed in any of the contemporary mereotopology endeavors. "Symmetric connectivity" can be retained by defining two things $\mathrm{a}, \mathrm{b}$ as isConnected if at least one path direction does exist. This allows for a more general - even symmetric - formulation:

$$
\mathrm{a}, \mathrm{b} \text { are connected } \Leftrightarrow \mathrm{a} \text { isPathConnected } \mathrm{b} \quad \vee \quad \mathrm{b} \text { isPathConnected } \mathrm{a}
$$

This expression, which still is symmetric, can be satisfied by following three configurations:

$$
\begin{array}{cccc}
\mathrm{a} \text { isPathConnected } \mathrm{b} & \wedge & \mathrm{b} \neg \text { isPathConnected } \mathrm{a} \\
\mathrm{a} \neg \text { isPathConnected } \mathrm{b} & \wedge & \mathrm{b} \text { isPathConnected } \mathrm{a} \\
\mathrm{a} \text { isPathConnected } \mathrm{b} & \wedge & \mathrm{b} \text { isPathConnected } \mathrm{a}
\end{array}
$$

The last expression indicates the existence of a reversible path, while both other expressions lead to irreversible situations, where there is a path from " $a$ " to " $b$ " (or vice versa) but no way back. Path connectivity is an important concept being introduced by Richard P. Feynman and finds applications in the principles of least action and/or Fermat's principle. It is discussed in a little more detail in section 5.5.

In a last but one step Axiom (C5) is discussed, which essentially states that if two things globally exist, they are connected: 


$$
\begin{gathered}
\Phi_{a} \neq 0 \wedge \Phi_{b} \neq 0 \rightarrow \Phi_{a} \Phi_{b} \neq 0 \\
\Phi_{a} \Phi_{b}=\frac{1}{N_{x}^{2}} \sum_{j=1}^{N_{x}} \Phi_{a}\left(x_{j}\right) \sum_{k=1}^{N_{x}} \Phi_{b}\left(x_{k}\right)=\frac{1}{N_{x}^{2}}\left(\sum_{\substack{k=1 \\
j=k}}^{N_{x}} \Phi_{a}\left(x_{j}\right) \Phi_{b}\left(x_{k}\right)+\sum_{\substack{j, k=1 \\
j \neq k}}^{N_{x}} \Phi_{a}\left(x_{j}\right) \Phi_{b}\left(x_{k}\right)\right) \neq 0
\end{gathered}
$$

The second sum contains correlations between the volume elements $x_{j}$ and $x_{k}$ of the reference frame when re-expressing the fields as products (i.e. $\Phi_{a}\left(x_{j}\right) \equiv$ $\Phi_{a} x_{j}$; see Appendix B5). Neglecting such correlations ${ }^{8}$ (i.e. $x_{j} x_{k}=0$ for all $\mathrm{i}, \mathrm{j}$ ) the second term on the RHS vanishes and only the first sum remains:

$$
\begin{gathered}
\Phi_{a} \Phi_{b} \neq 0 \rightarrow \sum_{k=1}^{N_{x}} \Phi_{a}\left(x_{k}\right) \Phi_{b}\left(x_{k}\right) \neq 0 \\
\sum_{k=1}^{N_{x}} \Phi_{a}\left(x_{k}\right) \Phi_{b}\left(x_{k}\right) \neq 0 \rightarrow \partial \Phi_{a, b} \neq 0 \\
\partial \Phi_{a, b} \neq 0 \rightarrow \Phi_{a} C \Phi_{b}
\end{gathered}
$$

Expressed in words this reads "If two things globally exist, they are connected". Axiom (C5) thus is recovered also by the phase field perspective for the case of exactly two coexisting things. However, for three (and more things) existing globally, i.e. for

$$
\Phi_{a} \neq 0 \wedge \Phi_{b} \neq 0 \wedge \Phi_{c} \neq 0
$$

a number of options occurs, which can be identified when using Axiom (C3):

$$
\begin{aligned}
\Phi_{a} C\left(\Phi_{b}+\Phi_{c}\right) \neq 0 \rightarrow & \Phi_{a} \Phi_{b}+\Phi_{a} \Phi_{c} \neq 0 \rightarrow \Phi_{a} \Phi_{b} \neq 0 \vee \Phi_{a} \Phi_{c} \neq 0 \\
& \rightarrow \Phi_{a} C \Phi_{b} \vee \Phi_{a} C \Phi_{c}
\end{aligned}
$$

Thus - even in case two things a and b do exist globally (i.e. have non-zero values) these two things a and $b$ do not necessarily need to be connected if more than two things are globally present. In case " $a$ " and " $b$ " not being mutually connected, both have to be connected to - or separated by - a third thing c. This directly implies the following global relations

$$
\begin{gathered}
\Phi_{a} \neq 0 \wedge \Phi_{b} \neq 0 \wedge \Phi_{c} \neq 0 \\
\rightarrow \Phi_{a, b} \neq 0 \vee \partial \Phi_{a, c} \neq 0 \vee \partial \Phi_{b, c} \neq 0
\end{gathered}
$$

Expressed in words this reads "If three things globally exist, each of them is connected to at least one of the two other things". While two things being mutually connected directly implies that both exist globally (see Axiom C1):

$$
\Phi_{a} C \Phi_{b} \rightarrow \sum_{k=1}^{N_{x}} \Phi_{a}\left(x_{k}\right) \Phi_{b}\left(x_{k}\right) \neq 0 \rightarrow \Phi_{a} \Phi_{b} \neq 0
$$

their individual global existence - in contrast however - does NOT imply that they are connected if more than two things are considered. In case of three things a,b,c two of them

8 In the spirit of the present article such correlations will exist between volumes being in contact with each other i.e between neighboring positions. They are neglected here to show under which conditions the axioms of Contact Algebra can be recovered. These terms open options for a future refinement of the concept. 
e.g. a,b may be mutually connected or may be disconnected (i.e. separated by the third thing $\mathrm{c}$ then):

$$
\begin{gathered}
\Phi_{a} \Phi_{b} \neq 0 \rightarrow \partial \Phi_{a, b} \neq 0 \vee \partial \Phi_{a, b}=0 \\
\partial \Phi_{a, b}=0 \rightarrow \Phi_{a} \neg C \Phi_{b}
\end{gathered}
$$

In case one of these boundaries does NOT exist (i.e. equals to 0) both other boundaries must exist (i.e. take non-zero values):

$$
\partial \Phi_{a, b}=0 \rightarrow \partial \Phi_{a, c} \neq 0 \wedge \partial \Phi_{b, c} \neq 0
$$

In the case a triple junction exists, all three dual boundaries do exist:

$$
\partial \Phi_{a, b, c} \neq 0 \rightarrow \partial \Phi_{a, b} \neq 0 \wedge \partial \Phi_{a, c} \neq 0 \wedge \partial \Phi_{b, c} \neq 0
$$

Axiom (C5) of Contact Algebra thus is recovered for exactly two existing things. The phase-field perspective depicted above seems however to imply that this axiom might have to be re-considered for the case of more than two things.

Eventually Axiom (C2) will be discussed. This seems the most complicated discussion as it involves 4 different things. The axiom (C2): "if $\mathrm{aCb}$ and $\mathrm{a} \leq \mathrm{c}$ and $\mathrm{b} \leq \mathrm{d}$, then $\mathrm{cCd}$ " expressed in words reads: If a isConnected $\mathrm{b}$ and a isPartOf $\mathrm{c}$ and if b isPartof $\mathrm{d}$ then $c$ isConnected $\mathrm{b}$. The individual expressions formulated in phase-field boundary terms translate into

$$
a C b \rightarrow \partial \Phi_{a, b} \neq 0
$$

If a isPartOf $\mathrm{c}$, there exists a boundary between a and c:

$$
a \leq c \rightarrow \partial \Phi_{a, c} \neq 0
$$

The total boundary of " $a$ " then hasPart the 2 dual boundaries, but inevitably then also comprises a triple junction $a, b, c$

$$
\partial \Phi_{a, \forall}=\partial \Phi_{a, b}+\partial \Phi_{a, c}+\partial \Phi_{a, b, c}
$$

Then further $\mathrm{b}$ isPart $\mathrm{f} \mathrm{d}$ implies the existence of a boundary between $\mathrm{b}$ and $\mathrm{d}$

$$
b \leq d \rightarrow \partial \Phi_{b, d} \neq 0
$$

The total boundary of " $\mathrm{b}$ " then hasPart the 2 dual boundaries, but inevitably also a triple junction $a, b, d$ :

$$
\partial \Phi_{b, \forall}=\partial \Phi_{b, a}+\partial \Phi_{b, d}+\partial \Phi_{a, b, d}
$$

If $\mathrm{aCb}$ then $\equiv \exists x_{n}$ such that $\partial \Phi_{a, \forall}\left(x_{n}\right) \neq 0 \wedge \partial \Phi_{b, \forall}\left(x_{n}\right) \neq 0$

$$
\partial \Phi_{a, b, d} \neq 0 \wedge \partial \Phi_{a, b, c} \neq 0 \rightarrow \partial \Phi_{a, b, c, d} \neq 0 \rightarrow \partial \Phi_{c, d} \neq 0 \rightarrow c C d
$$

Accordingly also Axiom C2 can be recovered by the phase field perspective.

In summary, all five axioms of the axiomatic system of contact algebra can be expressed in terms of dual and higher order boundaries as described by the phase-field perspective. 


\subsection{Comparison with Mereology}

While "connections" as used in Region Connect Calculus and topology have been described as "boundaries" between things in the preceding chapter, the description of a "part" being at the heart of mereology is related to the phase-field itself in the phase field perspective. The section starts with a short overview of mereology as being described in detail in [2].

\subsubsection{Mereological Axioms and Definitions}

Part: The monadic relation

$$
P x \equiv x \text { is A Part }
$$

defines " $x$ " to be a part. This expression implies the existence of " $x$ " as in order to be a part " $x$ " must exist. It finds its phase-field counterpart in the expression"

$$
\text { a is } A \text { Part } \equiv \Phi_{a} \neq 0
$$

which implies "a" (denoted as $\Phi_{a}$ in the phase-field perspective) to be a non-zero fraction of a system under consideration. The thing $\Phi_{\mathrm{a}}$ exists if it has a non-zero value, in case the thing does not exist it takes exactly the value 0 [46]:

$$
\begin{gathered}
\Phi_{a} \text { exists } \equiv \Phi_{a} \neq 0 \\
\Phi_{a} \text { notExists } \equiv \Phi_{a}=0
\end{gathered}
$$

Parthood: Mereology further builds on a dyadic relation specifying parthood:

$$
\text { Pxy } \equiv x \text { isPartofy }
$$

This parthood relation is considered as primitive following some basic axioms like (see e.g. [2]):

$$
\begin{aligned}
& P x x \quad \text { (Reflexivity) } \\
& P x y \wedge P y x \rightarrow x=y \quad \text { (Antisymmetry) } \\
& P x y \wedge P y z \rightarrow P x z \quad \text { (Transitivity) }
\end{aligned}
$$

Some commonly used definitions based on these axioms are

$$
\begin{array}{cc}
0 x y \equiv \exists z(P z x \wedge P z y) & (\text { Overlap }) \\
\mathrm{U} x y \equiv \exists z(P x z \wedge P y z) & \text { (Underlap) } \\
\mathrm{PP} x y \equiv \mathrm{P} x y \wedge \neg \mathrm{P} y x \quad \text { (ProperParthood) }
\end{array}
$$

Above axioms of Ground Mereology (M) are further complemented by a strong supplementation in Extensional Mereology (EM):

$$
\neg \mathrm{P} y x \rightarrow \exists z(P z y \wedge \neg O z x) \text { (Strong Supplementation) }
$$

Extensional Mereology then is further complemented by following closure extensional axioms leading to Closure Extensional Mereology (CEM):

$$
\begin{gathered}
\mathrm{U} x y \rightarrow \exists z \forall w(O w z \leftrightarrow(O w x \vee O w y)) \\
0 x y \rightarrow \exists z \forall w(P w z \leftrightarrow(P w x \wedge P w x)) \quad(\text { Product })
\end{gathered}
$$

9 by intention the phase field notation will deviate from using " $\mathrm{x}$ " to denote a part as usual in mereology. In the context of the phase field perspective, a,b,c etc will be used instead. This is to avoid confusion with the $x_{i}$ being used to denote elementary spatial regions in the phase field perspective. 


\section{$\exists z \forall x P x z \quad$ (Upper Bound)}

Specifying a particular, individual $\mathrm{z}$ matching the upper bound axiom fixes a universe under consideration having part all parts:

$$
u \equiv \exists ! z \forall x P x z \quad \text { (Universe) }
$$

\subsubsection{An essay towards a first order logic description of the phase-field concept}

The phase-field method to the best of knowledge of the author by now has not been formulated as an axiomatic system. The following section thus can be considered as a first essay towards formalizing the phase-field concept. It does not reveal the degree of maturity of the mereological axioms and is subject to future review. Before formulating the phase-field concept in FOL it is instructive to summarize the major concepts in their algebraic form.

In the phase-field concept all existing things are fractions and sum up to form the "whole" (i.e. the value 1). Not-existing things do not contribute to this sum as their values are identical 0 .

$$
\Phi_{0}+\sum_{i=1}^{N_{\Phi}} \Phi_{i}=1
$$

The "complement thing" $\Phi_{0}$ has been added to this sum to account for all un-named or un-identified - but existing - fractions of the universe. The "complement thing" accordingly can be defined as:

$$
\Phi_{0} \equiv 1-\sum_{i=1}^{N_{\Phi}} \Phi_{i}
$$

The result is the "basic equation" already being introduced in [1] with the summation starting from $\mathrm{i}=0$ :

$$
\sum_{i=0}^{N_{\Phi}} \Phi_{i}=1 \text { (basic equation) }
$$

Without rigorous proof - which is beyond the scope of the present article - a number of implications can directly be inferred:

(i) postulating the number of things to be finite $\left(\mathrm{N}_{\Phi}\right)$ directly implies the existence of a smallest fraction which has no parts (i.e. an "atom") and of a largest fraction (an "upper bound")

(ii) if a thing is the only thing (the "whole") it takes the value 1 and its complement is 0

(iii) if a thing is not the only thing, the complement exists (i.e. it has a non-zero value)

(iii) the basic equation corresponds to the mereological sum of all existing things (including the complement)

(v) in case both - a thing and its complement thing - exist, their -mereological- product (or their "boundary", or their "correlation") does exist and they are connected (see section 4.2):

$$
\Phi_{i} \neg \Phi_{i}=\Phi_{i}\left(1-\Phi_{i}\right) \neq 0
$$


Note that this correlation term between a thing and its complement is found in a number of areas. Examples are the logistic differential equation, where it corresponds to the derivative of the logistic function ${ }^{10}$ and is named the logistic distribution:

$$
f^{\prime}(x)=f(x)(1-f(x))
$$

The expression interestingly also corresponds to the lowest order Taylor approximation of entropy type terms [1]:

$$
\Phi_{i}\left(1-\Phi_{i}\right) \sim-\Phi_{i} \ln \Phi_{i}=S
$$

In the following a FOL description of above concepts is attempted. For this purpose the conventions of FOL are adapted and the $\Phi_{i}$ thus are denoted as x,y,z etc. again the following.

In the phase-field perspective all things - whether existing or non-existing (e.g. not yet or no more existing) - are fractions (of a whole):

\section{$\forall x \quad x \in \mathbb{Q}[0,1]$ (fractions)}

The closed interval $[0,1]$ here is the interval of rationale numbers $\mathbb{Q}[0,1]$, because any fraction by definition is a rationale number. In contrast to selecting the same interval of the real numbers $\mathbb{R}[0,1]$ this implies things to be countable as the set of rationale numbers is countable. In a refined axiomatization even a finite cardinality of the collection of $\mathrm{N}$ things could be postulated. In case a thing exists it takes a non-zero value, in case it does not exist it takes the value 0 :

$$
\begin{gathered}
\exists x \equiv x \neq 0 \quad(\text { existence }) \\
\neg \exists x \equiv x=0 \quad(\text { non }- \text { existence }) \\
\exists x \leftrightarrow \quad x \in(0,1] \\
\exists x \leftrightarrow x=1 \vee(0<x<1)
\end{gathered}
$$

This provides a link between "Boolean Logics" and more general types of logic like Heyting logic ${ }^{11}$ or Fuzzy logic ${ }^{12}$ allowing for multiple logic states beyond the binary Boolean alternative of "true" and/or "false". The Boolean view is recovered if selecting the values from the interval of the natural numbers $\mathbb{N}[0,1]$ which only has the two elements 0 and 1 instead of $\mathbb{Q}[0,1]$ :

The above relation

$$
\begin{aligned}
& \text { "true" (or Boolean I) translates into " } \neq 0 " \\
& \text { "false" (or Boolean 0) translates into } "=0 "
\end{aligned}
$$

$$
\exists x \leftrightarrow x=1 \vee(0<x<1)
$$

expressed in words reads: if $x$ exists (i.e. has a non zero value) it is either the whole (with value 1) or a part (with value beween 0 and 1). This allows for the specification of whole and of part in the following.

The whole corresponds to a unique, single object (universe) with no further objetcs being existing then:

$\exists x(x=1)$ (Whole)

$x=1 \rightarrow \forall y(\neg \exists y)$ (no further thing)

10 https://en.wikipedia.org/wiki/Logistic_function

11 https://en.wikipedia.org/wiki/Intuitionistic_logic

12 https://en.wikipedia.org/wiki/Fuzzy_logic 
The monadic part relation as used e.g. in mereology is recovered by specifying "parts" as existing fractions (of a whole) with values smaller one:

$$
P x \equiv x<1 \wedge \exists x \quad(\text { Part })
$$

which is equivalent to $x$ having a value in the open interval $(0,1)$ :

$$
P x \leftrightarrow x \in(0,1)
$$

If $\mathrm{x}$ is a part or $\mathrm{x}$ does not exist, there exists at least one other fraction/thing:

$$
P x \vee \neg \exists x \rightarrow \exists y((x+y<1) \vee(x+y=1)) \quad \text { (General Supplement) }
$$

In case $\mathrm{x}$ is a part the "other" thing $\mathrm{y}$ is also a part:

$$
P x \rightarrow \exists y \wedge(x+y \leq 1) \rightarrow \exists y \wedge y<1) \rightarrow P y
$$
whole:

These two fractions either supplement each other to form a part, which still is not the

$$
\begin{gathered}
x<1 \rightarrow \exists y(x+y<1)(\text { Supplement }) \\
x<1 \rightarrow \exists y(y<\neg x)
\end{gathered}
$$

or the two fractions $x$ and $y$ complement each other to form the whole:

$$
\begin{gathered}
\neg x \equiv \exists y(x+y=1)(\text { Complement }) \\
\neg x \equiv \exists y(y=1-x) \\
\neg x \equiv(1-x)
\end{gathered}
$$

The thing and its complement always complement each other to form the universe:

$$
\begin{gathered}
\forall x(x+\neg x=1) \text { (Universal Union) } \\
\text { which compares to } \\
\forall x(x \vee \neg x=\text { true })(\text { Boolean })
\end{gathered}
$$

The Universal Union allows inferring, that the whole has no complement:

$$
x=1 \rightarrow \neg x=0 \rightarrow \neg \exists \neg x \text { (Whole has no complement ) }
$$

In contrast to Boolean logic a thing and its complement can coexist, i.e. they both can have non-zero values:

$$
\forall x: P x \rightarrow(x \wedge \neg x \neq 1)(\text { Coexistence })
$$

This expression decomposes into two cases:

$$
x \wedge \neg x \neq 1 \rightarrow((x \wedge \neg x=0) \vee(x \wedge \neg x \neq 0))
$$

Case a) $(x \wedge \neg x=0)$

For a binary interval of the natural numbers - i.e. the Boolean case - the only alternative for selecting "not being equal 1 " is to be equal 0 . Case a) thus reflects the Boolean view that a thing and its complement do not coexist:

$$
\forall \mathrm{x}: \mathrm{x} \wedge \neg \mathrm{x}=0 \text { (false) }
$$

The Boolean view thus is recovered in this special case. From the phase-field logic this expression reads

$$
\begin{gathered}
(x \wedge \neg x=0) \rightarrow \neg \exists(x \wedge \neg x) \rightarrow \neg \exists x \vee \neg \exists \neg x \\
\rightarrow x=0 \vee \neg x=0 \rightarrow x \neg x=0
\end{gathered}
$$

Expressed in words this reads that a thing and its complement do not coexist if either the thing or its complement do not exist individually. 


\section{Case b) $(x \wedge \neg x \neq 0)$}

Case b) is possible in a non-Boolean perspective only. In the phase-field formulation one gets

$$
\begin{gathered}
(x \wedge \neg x \neq 0) \rightarrow \exists x \wedge \exists \neg x \\
\rightarrow x \neq 0 \wedge \neg x \neq 0 \\
\rightarrow x \neg x \neq 0
\end{gathered}
$$

Note that $\neg \mathrm{x}$ does NOT mean that $\mathrm{x}$ does not exist but $\neg \mathrm{x}$ denotes the complement of $x$. In contrast: if $x$ does not exist its complement does exist and vice versa via the GeneralSupplement Axiom:

$$
\begin{aligned}
& \neg \exists x \rightarrow \exists \neg x \\
& \neg \exists \neg x \rightarrow \exists x
\end{aligned}
$$

In the phase field perspective thus anything (!) which is a part overlaps (i.e. isConnectedTo) its complement part:

$$
O x \neg x \equiv \forall x(\mathrm{Px} \wedge P \neg x)(\text { Fundamental Overlap })
$$

This fundamental overlap is given by the algebraic product of the two non-zero fractions of the thing and its complement:

$$
\begin{gathered}
O x \neg x \equiv x \neg x \\
\rightarrow O x \neg x=x(1-x))
\end{gathered}
$$

The general overlap between two parts is defined as

$$
\begin{aligned}
O x y \equiv & (\mathrm{Px} \wedge \mathrm{P} y)(\text { Overlap }) \\
& \rightarrow O x y=x y
\end{aligned}
$$

The overlap in phase-field perspective corresponds to the situation of two things being connected, being collocated resp. two things having a boundary. Any part is connected to its complement in view of the Fundamental Overlap. If the complement of $\mathrm{x}$ has two parts $\mathrm{y}$ and $\mathrm{z}, \mathrm{x}$ is connected to at least one of them (see also discussion of Axiom C3 of contact algebra in section 4.2):

$$
\begin{gathered}
O x \neg x \wedge(\mathrm{P} \neg x=P y \vee P z) \rightarrow \mathrm{Px} \wedge(\mathrm{P} y \vee P z) \\
\mathrm{Px} \wedge(\mathrm{P} y \vee P z) \leftrightarrow(\mathrm{Px} \wedge \mathrm{P} y) \vee(\mathrm{Px} \wedge P z) \\
O x \neg x=\mathrm{xy} \vee \mathrm{xz}
\end{gathered}
$$

A full FOL (First-Order $\operatorname{Logic}^{13}$ ) or IPL (intuitionistic propositional logic ${ }^{14}$ ) description of the phase-field concept is beyond the scope of the present article and will be subject of a future separate publication. It will include the definition of objects like triple \& quadruple junctions and interesting objects like the ratio of things.

\subsubsection{Further useful definitions}

The following section defines some further objects based on the Closure Extensional Mereology (CEM) framework described in section 4.3.1. The definition of these objects is helpful for the comparison with the phase-field perspective. The three objects being discussed for this purpose are the self-sum, the triple overlap and the triple product.

\footnotetext{
${ }^{13}$ https://en.wikipedia.org/wiki/First-order_logic

${ }^{14}$ https://en.wikipedia.org/wiki/Intuitionistic_logic
} 
Self-Sum: Formally interpreting the minimal underlap (i.e. the mereological sum) of a thing with itself leads to following specification ${ }^{15}$ :

This expression reduces to

$$
\mathrm{U} x x \rightarrow \exists z \forall w(O w z \leftrightarrow(O w x \vee O w x))
$$

$$
\mathrm{U} x x \rightarrow \exists z \forall w(O w z=O w x) \quad(\text { Self }- \text { Sum })
$$

This "self-sum" will be shown to be identical with the fraction the part takes of the whole in the phase field perspective. ological sum

Triple Overlap: A closer look at the "equivalence" in the expression for the mereunveils three different options for Owz to be "true":

$$
\begin{aligned}
& \text { Owx }=\text { true } \wedge \text { Owy = false } \\
& \vee O w x=\text { false } \wedge \text { Owy = true } \\
& \vee O w x=\text { true } \wedge \text { Owy = true }
\end{aligned}
$$

The last expression suggests - and allows for - a definition of a triple junction in form of a triadic relation ${ }^{16}$ :

$$
\text { Тxyz } \equiv \exists w(O w z \wedge O w x \wedge O w y) \quad \text { (Triple Overlap) }
$$

Expressed in words this definition reads: "There exists a region $\mathrm{w}$ which overlaps with three regions $x, y, z$ ". This region is denoted as a "triple overlap".

Triple Product: Further a maximum triple overlap - a "triple product" - comprising all regions $w$ with triple overlaps of the three same three things can be defined:

$$
\mathrm{T} x y z \equiv \exists t \forall w(O w z \wedge O w x \wedge O w y) \quad \text { (Triple Product) }
$$

The definitions of triple product and triple overlap are amended to classical mereology here, as they find their counterparts in the phase-field perspective (see table in section 3.4).

\begin{tabular}{|c|c|c|c|c|c|}
\hline & $\mathrm{U} x_{n} y$ & $\mathrm{O} x_{n} y$ & $\mathrm{PP} x_{n} y$ & $\mathrm{EQ} x_{n} y$ & $\mathrm{PE} x_{n} y$ \\
\hline$n=1$ & + & - & - & - & - \\
\hline$n=2$ & + & + & - & - & - \\
\hline$n=3$ & + & + & + & - & - \\
\hline$n=4$ & + & + & - & + & - \\
\hline$n=5$ & + & + & - & - & + \\
\hline
\end{tabular}

\subsubsection{Graphical visualisation of mereological expressions}

Before eventually discussing mereology from the phase-field perspective, a graphical visualization of the different definitions in mereology is very instructive. Numerous graphical representations of the classical definitions and relations are available, e.g. Fig.12.

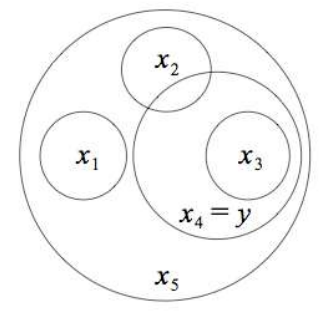

Figure 12: Overview of mereological relations (underlap, overlap, proper parthood equality and enclosure) between different things. [https://plato.stanford.edu/entries/mereology/].

15 This formulation by now has not been used in mereology formulations to the best of the authors knowledge.

16 This definition is not part of any mereology formulation by now. It is introduced here for the first time. 
Some further graphics are added in the following allowing discussing some of the terms in more detail or trying to illustrate some of the terms graphically at all.

Underlap: The mereological underlap Uxy denotes a thing $\mathrm{z}$ which has two things $\mathrm{x}$ and $y$ as parts. The two extremes are the whole (i.e. the universe individual) representing the maximum object comprising both things and the mereological sum representing the minimum object comprising both things, Figure 13.
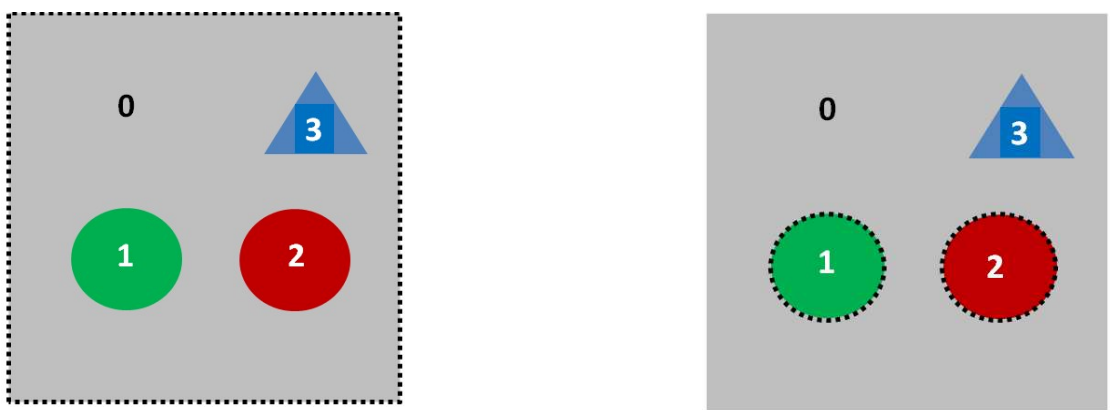

Fig. 13: The "universe individual" (left, marked by its dashed boundary) is one of the possible selections for an underlap of the two things1\&2, as per definition the universe hasPart all things. Another possible selection is the mereological sum representing the minimum object comprising both things (right, boundaries also marked by dashed lines)

However, there is no "unique underlap". A variety of different objects fulfil the condition to be an underlap of two things. Between the two extremes depicted in Fig.13, a variety of different underlap objects can accordingly be defined, Fig. 14.

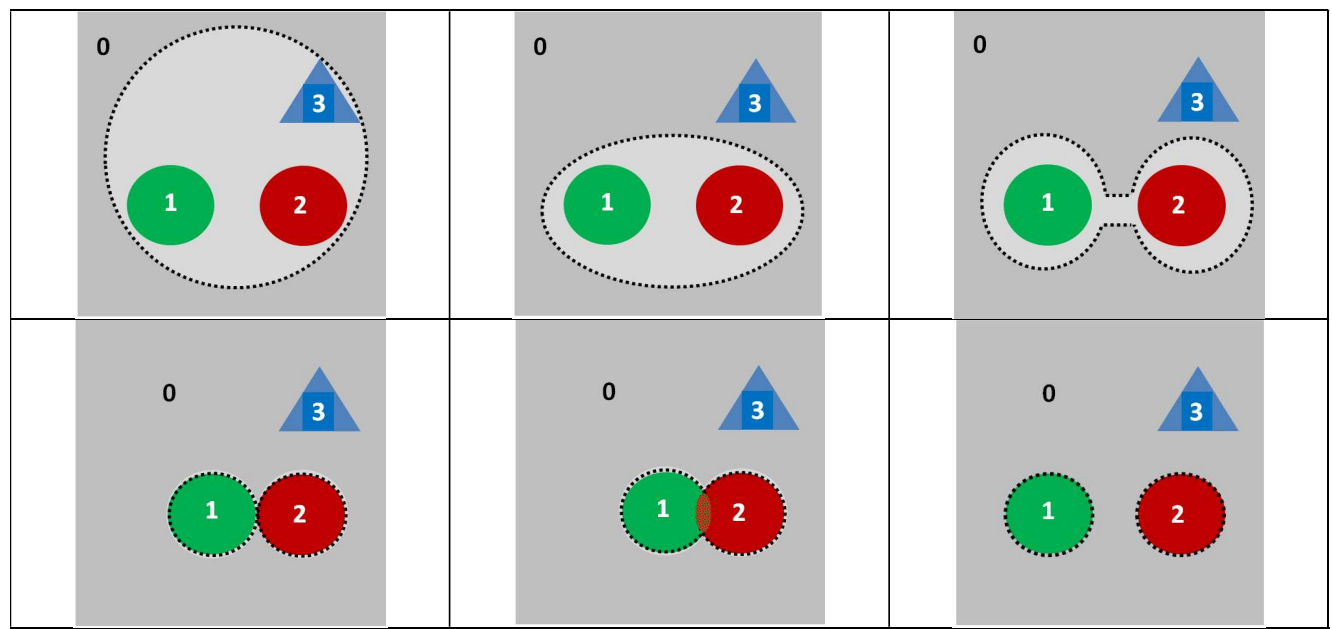

Fig.14: Different possible underlaps (light grey) for two things (1/green, $2 /$ red) being part of a universe individual (dark grey) having three things $(1,2,3)$ and a matrix thing $(0)$ as parts. Note that the underlap in all cases, except for the lower right case, is a single, self-connected object. The lower right case corresponds to the mereological sum of two disconnected things. Even for disconnected things the underlap may however be self-connected (see text). The different possible self-connected underlaps depicted in this figure differ in their size and thus allow for a contineous description of a transition from "isDisconnected" via "isExternalContact" to "isPartOf" with a variable describing this

transition being the "fraction" the underlap takes of the universe (see text). The mereological sum of two disconnected parts does not fit into such a sequence as itself is not self-connected. The mereological sum of two connected parts, in contrast, fits well into the sequence as it is a self-connected object. 


\section{Self-connected underlaps of disconnected parts}

In case the underlap is postulated to be self-connected for both connected and also for disconnected things, a minimum of such a self-connected underlap can be used as a measure for distance, Fig. 15. Further separating the two things will increase this minimal volume, while approaching them will decrease it. A distance d, which also is a fraction of the universe, can thus be defined as the difference of the minimum self-connected underlap "MSCU" region and the "Mereological Sum"(MS, which is not self-connected for disconnected things).The value of $\mathrm{d}$ will become 0 in the case of external contact between the two things.

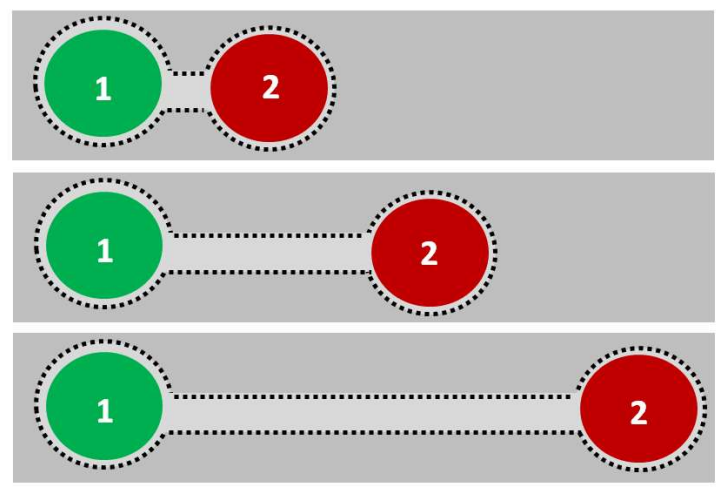

Fig. 15: Schematic sketch of two things being separated by different "distances" (increasing from top to bottom). While the boundary between most of part 1 and the underlap and most of part 2 of the underlap is minimized and will essentially not depend on their relative position, the volume of a connecting "string", "tube" or "path" will essentially linearly depend on the distance.

The volume of the minimum self-connected underlap (MSCU) will correspond to the sum of the underlaps of the individual parts (i.e. their mereological sum) plus a volume corresponding to the minimal string/path connecting the two parts (the path volume; see also section 5.6) minus the total volume of their overlap (i.e. their mereological product). This concept is not addressed in current mereology, but can most probably be related to a concept of "potential energy". The further discussion of this idea is beyond the scope of the current article and will be subject of future work.

\subsubsection{The phase-field perspective of mereological expressions}

An intuitive approach to a translation of mereological expressions to their phase-field counterparts starts from the mereological definition of the overlap:

$$
0 x y \equiv \exists z(P z x \wedge P z y) \quad(\text { Overlap })
$$

The overlap in the phase field perspective corresponds to the coexistence of the object $\Phi$ (denoted as $x$ in the mereology expression) and an object of the reference frame $\mathrm{x}_{\mathrm{n}}$ (denoted $\mathrm{y}$ in the mereology expression). In case of coexistence, both things are non-zero fractions of a universe i.e. both have values in the open interval $(0,1)$ of the rationale numbers. Their algebraic product (their correlation, " $z$ " in the mereology expression i.e. the overlap) thus exists:

$$
\Phi \mathrm{x}_{n} \neq 0
$$

This allows defining the phase-field (or even any scalar field) as being the overlap of an object $\Phi$ and an object $x_{n}$ being part of a reference frame. Without loss of generality, $\mathrm{X}_{\mathrm{n}}$ can exemplarily be imagined as a simple, individual voxel in a cubic grid.

$$
\left.\Phi\left(\mathrm{x}_{n}\right) \equiv \Phi \mathrm{x}_{n} \quad \text { (phase }- \text { field }\right)
$$


The mereological self-sum, which was introduced as special case of the mereological sum in section 4.3.3, can then be used to identify the total fraction of the object in the entire reference frame being composed of "all $\mathrm{xn}$ "

$$
\mathrm{U} x x \rightarrow \exists z \forall w(O w z=O w x) \quad(\text { self }- \text { sum })
$$

Identifying "all $\mathrm{x}_{\mathrm{n}}$ " (phase-field perspective) with "all $\mathrm{w}$ " (mereology) and $\mathrm{x}$ (mereology) with the object $\Phi$ (phase-field) facilitates the translation between the phase-field perspective and mereological expressions. The object $\mathrm{z}$ in this case is the total fraction of the object $\Phi$. Assuming further "all $\mathrm{x}_{\mathrm{n}}$ " and thus "all $\mathrm{w}$ " to be countable and finite allows specifying the total fraction $\Phi$ the object, which sums all $\mathrm{x}_{\mathrm{n}}$ where $\Phi$ is present.

$$
\Phi=\frac{1}{N_{x}} \sum_{n=1}^{N_{x}} \Phi\left(\mathrm{x}_{n}\right)
$$

\section{Uxx translates into $\Phi$ which is the global fraction of the object $\Phi$}

Having thus related the phase-field expressions $\Phi\left(\mathrm{x}_{\mathrm{n}}\right)$ and $\Phi$ (see equation 1 in table in section 3.6) to mereological expressions, in the next steps the two expressions $\partial \Phi_{i, k}$ and $\partial \Phi_{i, k}\left(x_{n}\right)$ (see equation 3 in table in section 3.6) will be discussed. They can be identified to relate to the mereological product. The mereological product is the largest overlap of two phase fields describing two things a and $b$, i.e. the region formed by all $\mathrm{x}_{\mathrm{n}}$ where the two things coexist ${ }^{17}$. The smallest region of coexistence - the smallest overlap - is defined by coexistence of the two things at least in a single $\mathrm{x}_{\mathrm{n}}$ :

$$
O a b \equiv \exists x_{n}\left(\Phi_{a}\left(x_{n}\right) \Phi_{b}\left(x_{n}\right) \neq 0\right)
$$

Expressed words: There exists a volume $\mathrm{x}_{\mathrm{n}}$ which hasPart finite fractions of both parts $a$ and $b$ (or which isPart of both $a$ and $b$ ):

$$
\mathrm{Oab} \equiv \exists x_{n}\left(\mathrm{Px}_{n} a \wedge \mathrm{Px}_{n} \mathrm{~b}\right)
$$

This exactly is the mereological definition of overlap and also to the phase-field definition of an interface in an elementary volume of the reference frame:

$$
\partial \Phi_{a, b}\left(x_{n}\right) \neq 0
$$

Oab translates into $\partial \Phi_{a, b}\left(x_{n}\right)$ which is the local fraction of the boundary between $a$ and $b$

The maximum overlap - i.e. the mereological product- is the object comprising all $\mathrm{x}_{\mathrm{n}}$ which contribute to the total boundary between the two things a and $b$ :

$$
\partial \Phi_{a, b}=\frac{1}{N_{x}} \sum_{n=1}^{N_{x}} \partial \Phi_{a, b}\left(x_{n}\right)
$$

The mereological product Oab translates into $\partial \Phi_{a, b}$ which is the total fraction the boundary between $a$ and $b$ takes in the universe under consideration

\footnotetext{
17 Things have been named a \& b here in order not to generate confusion with the $x$ denoting an existing object of the reference frame in the phase field perspective.
} 
Recovering the definition of the phase-field as the correlation (overlap) between the thing and a VolumeElement of a reference frame (see Appendix B):

$$
\Phi_{a}\left(\mathrm{x}_{n}\right) \equiv \Phi_{a} \mathrm{x}_{n} \quad \text { resp. } \quad \Phi_{b}\left(\mathrm{x}_{n}\right) \equiv \Phi_{b} \mathrm{x}_{n}
$$

allows rewriting $O a b \equiv \Phi_{a} \mathrm{x}_{n} \Phi_{b} \mathrm{x}_{n} \neq 0$ which eventually can be interpreted as a triadic relation : Tabx $\equiv \Phi_{a} \mathrm{x}_{n} \Phi_{b} \neq 0$. Expressed in words this triadic relation reads: Tabx: $a \mathcal{E} b$ collocate in $x$. It can likewise be formulated for coexistence as Tabt: $a \mathcal{E} b$ coexist during $t$. Eventually a quartic relation Qabxt for a physical contact, which corresponds to coexistence (during $\mathrm{t}$ ) and collocation (in $\mathrm{x}$ ) can be formulated: Qabxt: $a \mathcal{E} b$ coexist in $x$ during $t$

The Mereological sum - i.e. a possible, minimum thing c having part two things a and bfrom a phase-field perspective can directly be identified as the sum of the two individual phase-fields describing the two things $a$ and $b$ coexisting in an $x_{n}$. The thing $c-$ the sum - is described by its own phase-field then only has " $a$ " and " $b$ " as parts and no further thing:

$$
\Phi_{c}\left(x_{n}\right)=\Phi_{a}\left(x_{n}\right)+\Phi_{b}\left(x_{n}\right)
$$

Uab (Underlap) is the same as $\Phi_{a}\left(x_{n}\right)+\Phi_{b}\left(x_{n}\right)$ (sum of local fractions)

$$
\Phi_{c}=\frac{1}{N_{x}} \sum_{n=1}^{N_{x}}\left(\Phi_{a}\left(x_{n}\right)+\Phi_{b}\left(x_{n}\right)\right)=\Phi_{a}+\Phi_{b}
$$

Uab (Mereological sum) is the same as $\Phi_{a}+\Phi_{b} \quad$ (sum of global fractions)

\subsubsection{Graphical comparison of mereological and phase-field descriptions}

Boundary "areas" in mereotopology MT and in the Region Connect Calculus RCC correspond to Overlaps. External contact (EC) and tangential proper parts (TPP) both relate to triple junctions. There is no equivalent to quadruple junctions provided in either of these two concepts. The phase field approach allows the description of mereotopological relations between things on the basis of the boundaries and the higher order junctions they form, Figs. 16 and 17. Examples for three or more things forming a whole are depicted in Fig.18.

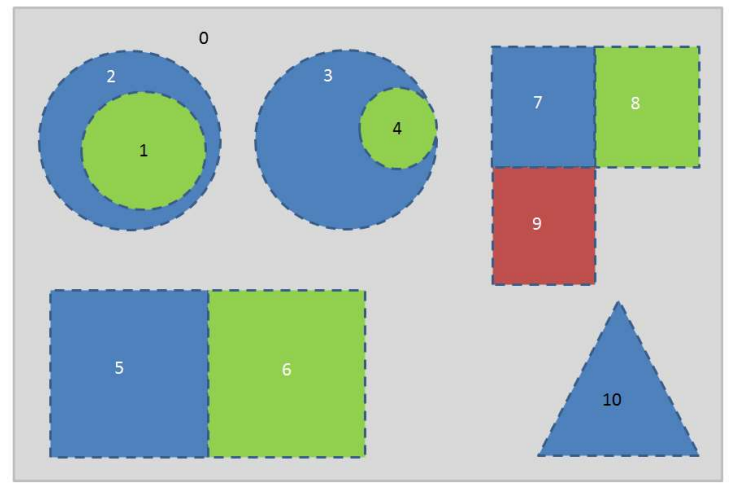

Figure 16: Different configurations of things forming a variety of interfaces, triple lines and quadruple points. The total boundary of thing 7 $\partial \Phi_{7, \forall}$ consists of boundary regions with the matrix 0 , and with things 8 \& 9 and also includes triple and quadruple junctions meaning that $\partial \Phi_{7, \forall}=\partial \Phi_{7,0}+\partial \Phi_{7,8}+\partial \Phi_{7,9}+$ $\partial \Phi_{7,8,0}+\partial \Phi_{7,9,0}+\partial \Phi_{7,9,8,0}$ (see text for the other objects)

Based on boundaries, the topological relations between the things being depicted in Figure 17 can easily described. For example thing 10 has an interface with the matrix 0 
only: $\partial \Phi_{10, \forall}=\partial \Phi_{10,0}$. Thing 1 is proper part of thing 2 and thus has an interface with 2 only: $\partial \Phi_{1, \forall}=\partial \Phi_{1,2}$. Thing 2 (in absence of thing 1 ) is a direct part of the universe (Thing 0) and thus has an interface with 0 only: $\partial \Phi_{2, \forall}=\partial \Phi_{2,0}$. In presence of thing 1 , however, thing 2 has above external boundary with thing 0 but also a further internal boundary with thing 1: $\partial \Phi_{2, \forall}=\partial \Phi_{2,0}+\partial \Phi_{2,1}$. Thing 4 is tangential part of thing 3 and thus has an interface with 3 only but also a single triple-junction: $\partial \Phi_{4, \forall}=\partial \Phi_{4,3}+$ $\partial \Phi_{4,3,0} \partial \Phi_{4,0,3}$. Things $5 \& 6$ represent a "bound state" and their boundaries are $\partial \Phi_{5, \downarrow}=\partial \Phi_{5,0}+\partial \Phi_{5,6}+\partial \Phi_{5,6,0}+\partial \Phi_{5,0,6}$ and $\partial \Phi_{6, \downarrow}=\partial \Phi_{6,0}+\partial \Phi_{6,5}+\partial \Phi_{6,5,0}+\partial \Phi_{6,0,5}$. 


\begin{tabular}{|c|c|c|c|}
\hline \multicolumn{2}{|c|}{$\begin{array}{l}\text { mereotopology } \\
\text { description }\end{array}$} & $\begin{array}{l}\text { configuration } \\
\text { (geometric) }\end{array}$ & $\begin{array}{l}\text { boundary description } \\
\text { (this article) }\end{array}$ \\
\hline $\begin{array}{c}\text { IOxy } \\
\text { Uxy } \\
\text { (IUxy) }\end{array}$ & $\begin{array}{l}\text { IOyx } \\
\text { Uyx } \\
\text { (IUyx) } \\
\text { IPPyx }\end{array}$ & & $\begin{array}{c}\partial \Phi_{1, \forall}=\partial \Phi_{1,0}+\partial \Phi_{1,2} \\
\partial \Phi_{2, \forall}=\partial \Phi_{2,1} \\
\partial \Phi_{0, \forall}=\partial \Phi_{0,1}+\text { external }\end{array}$ \\
\hline \multicolumn{4}{|c|}{$\begin{array}{l}\text { Case \#1: part } 2 \text { ("y", yellow) is internal proper part of part } 1 \text { (" } x \text { " blue). } 2 \text { is ConnectedTo (i.e. has boundary with) } \\
1 \text { only, while } 1 \text { has a boundary with } 0 \text { in addition. The matrix has an "external" boundary (dashed grey) }\end{array}$} \\
\hline $\begin{array}{l}\text { IOxy } \\
\text { Uxy } \\
\text { (IUxy) } \\
\text { (TUxy) }\end{array}$ & $\begin{array}{l}\text { IOyx } \\
\text { Uyx } \\
\text { (IUyx) } \\
\text { (TUyx) } \\
\text { TPPyx }\end{array}$ & & $\begin{array}{c}\partial \Phi_{1, \forall}=\partial \Phi_{1,2}+\partial \Phi_{1,0}+\partial \Phi_{1,2,0}+\partial \Phi_{1,0,2} \\
\partial \Phi_{2, \forall}=\partial \Phi_{2,0}+\partial \Phi_{2,1}+\partial \Phi_{2,1,0}+\partial \Phi_{2,0,1} \\
\partial \Phi_{0, \forall}=\partial \Phi_{0,1}+\partial \Phi_{0,2}+\partial \Phi_{0,1,2}+\partial \Phi_{0,2,1}+e x t \\
\partial \Phi_{1,0}>\partial \Phi_{2,0} \\
\partial \Phi_{2,1}>\partial \Phi_{2,0}\end{array}$ \\
\hline
\end{tabular}

Case \#2: Both things have a mutual boundary. Both things (i) have a boundary with " 0 " and (ii) have two triple junctions. The total boundary of 1 with 0 is greater than the boundary of 2 with 0 .

\begin{tabular}{|c|c|c|c|}
\hline $\begin{array}{c}\text { IOxy } \\
\text { (IUxy) } \\
\text { (TUxy) }\end{array}$ & $\begin{array}{c}\text { IOyx } \\
\text { (IUyx) } \\
\text { (TUyx) }\end{array}$ & & $\begin{array}{c}\partial \Phi_{1, \forall}=\partial \Phi_{1,2}+\partial \Phi_{1,0}+\partial \Phi_{1,2,0}+\partial \Phi_{1,0,2} \\
\partial \Phi_{2, \forall}=\partial \Phi_{2,0}+\partial \Phi_{2,1}+\partial \Phi_{2,1,0}+\partial \Phi_{2,0,1} \\
\partial \Phi_{0, \forall}=\partial \Phi_{0,1}+\partial \Phi_{0,2}+\partial \Phi_{0,1,2}+\partial \Phi_{0,2,1}+e x t \\
\partial \Phi_{1,0}>\partial \Phi_{2,0} \\
\partial \Phi_{2,}<\partial \Phi_{20}\end{array}$ \\
\hline
\end{tabular}

Case \#3: This case is topologically identical with case \#2 from the phase field perspective in the sense that it reveals the same boundaries and the same number of triple junctions. It differs in the relative size of the boundaries with the boundary between 2 and 0 being larger as compared to case \#2. The fraction the boundary volume takes in this case is finite and not negligible.

\begin{tabular}{|c|c|c|}
\hline $\begin{array}{l}\text { TOxy } \\
\text { (IUxy) } \\
\text { (TUxy) }\end{array}$ & $\begin{array}{l}\text { TOyx } \\
(\mathrm{IUyx}) \\
\text { (TUyx) }\end{array}$ & $\begin{array}{c}\partial \Phi_{1, \forall}=\partial \Phi_{1,2}+\partial \Phi_{1,0}+\partial \Phi_{1,2,0}+\partial \Phi_{1,0,2} \\
\partial \Phi_{2, \forall}=\partial \Phi_{2,0}+\partial \Phi_{2,1}+\partial \Phi_{2,1,0}+\partial \Phi_{2,0,1} \\
\partial \Phi_{0, \forall}=\partial \Phi_{0,1}+\partial \Phi_{0,2}+\partial \Phi_{0,1,2}+\partial \Phi_{0,2,1}+\text { ext. }\end{array}$ \\
\hline
\end{tabular}

Case \#4: This case again is topologically identical with case \#2 and \#3 from the phase field perspective in the sense that it reveals the same boundaries and the same number of triple junctions. The volume the boundary takes is neglected here.

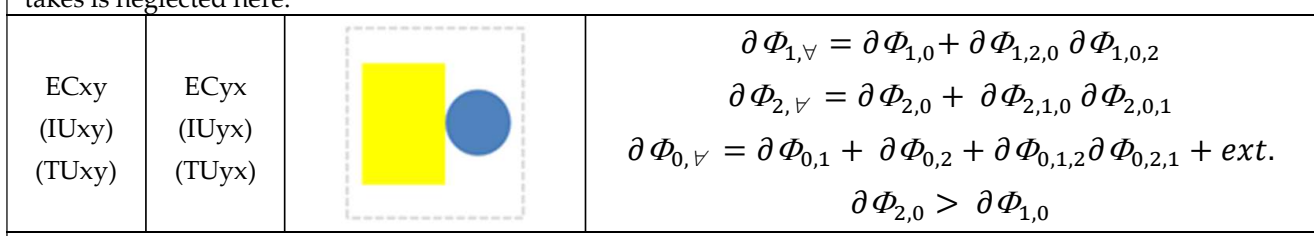

Case \#5: ExternalContact at a single triple point.This situation is decribed by two collocated (i.e. correlated) triple junctions

\begin{tabular}{|c|c|c|c|c|}
\hline \multirow{2}{*}{ DCxy } & DCyx & & & \\
& & & & $\partial \Phi_{1, \forall}=\partial \Phi_{1,0}$ \\
& & $\partial \Phi_{2, \forall}=\partial \Phi_{2,0}$ \\
& & $\partial \Phi_{0, \forall}=\partial \Phi_{0,1}+\partial \Phi_{0,2}+$ ext. \\
& $\partial \Phi_{2,0}>\partial \Phi_{1,0}$ \\
\hline
\end{tabular}

Case \#6: Disconnected parts. No common boundary. No triple junctions. Both things have a boundary with " 0 " only. The boundary of 2 (the yellow thing) with 0 is larger as compared to the boundary of 1 with 0 .

Figure 17: Comparison of mereological configurations with the phase-field perspective 


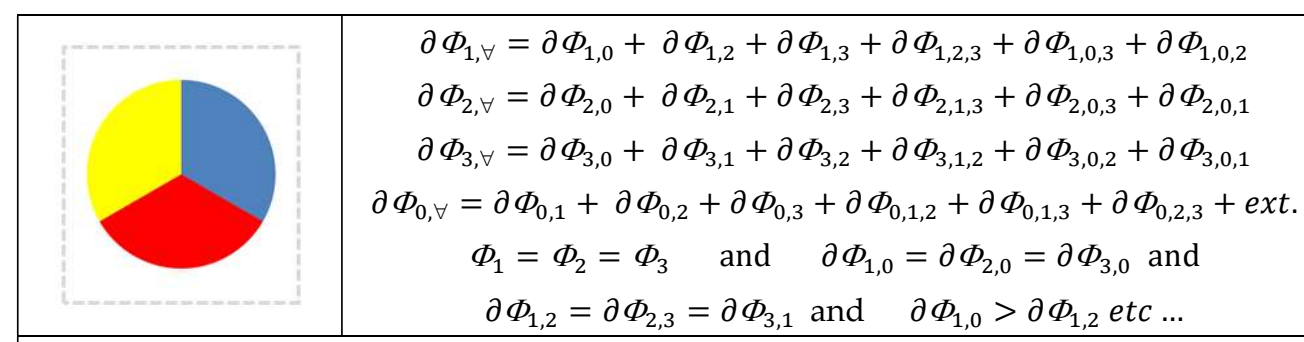

Case \#9: Add third part (1 blue 2 yellow 3, red, 0 Matrix): Above arrangement comprises 4 triple junctions, of which are 3 with vacuum/matrix and only one amongst the three parts.

$$
\begin{gathered}
\text { 0: gray, 1: white, 2: black, 3: white and 4: black } \\
\partial \Phi_{1, \forall}=\partial \Phi_{1,0}+\partial \Phi_{1,2}+\partial \Phi_{1,4}+\partial \Phi_{1,2,0}+\partial \Phi_{1,0,2} \\
\partial \Phi_{2, \forall}=\partial \Phi_{2,0}+\partial \Phi_{2,1}+\partial \Phi_{2,3}+\partial \Phi_{2,1,0}+\partial \Phi_{2,0,1} \\
\partial \Phi_{3, \forall}=\partial \Phi_{3,2} \\
\partial \Phi_{4, \forall}=\partial \Phi_{4,1} \\
\partial \Phi_{0, \forall}=\partial \Phi_{0,1}+\partial \Phi_{0,2}+\partial \Phi_{0,1,2}+\partial \Phi_{0,2,1}+\text { ext. } \\
\Phi_{1}=\Phi_{2} \wedge \Phi_{3}=\Phi_{4} \\
\partial \Phi_{1,0}=\partial \Phi_{2,0} \wedge \partial \Phi_{1,4}=\partial \Phi_{2,3} \\
\partial \Phi_{1,0}>\partial \Phi_{1,4}
\end{gathered}
$$

Case \#10: Demonstration of the expressiveness of the concept depicted in the present article. Try to compose an item comprising 4 things $(1,2,3,4)$ and the background thing $(0$, gray, already placed) based on the information given above (solution and further discussion in Appendix D)

Figure 18: Some examples for the phase-field perspective of wholes comprising more than 2 parts (excluding the matrix)

\section{Extended notions of the isConnected relation}

Physics (from Ancient Greek: $\varphi v \sigma \iota \kappa \eta$ ) is the natural science that studies matter, its motion and behavior through space and time. A concept of mereology/mereotopology addressing space AND time - i.e. a 4D mereotopology - similar to the MereoGeometry of 3D static structures - might thus be named MereoPhysics [1].

The phase field $\Phi$ in its typical applications is not only a function of space but a function of both space and time:

$$
\Phi_{k} \text { exists at } x_{j} \text { during } t_{i} \rightarrow \exists t_{i}, x_{j} \wedge \Phi_{k}\left(x_{j}, t_{i}\right) \neq 0
$$

This allows for a phase-field based definition of „isConnected“(in 4D): $\Phi_{1}$ isConnected (in4D) to $\Phi_{2}$ if their correlation function is non-zero:

$$
\Phi_{1}\left(x_{1}, t_{1}\right) \text { isConnected } \Phi_{2}\left(x_{2}, t_{2}\right) \rightarrow \Phi_{1}\left(x_{1}, t_{1}\right) P_{2}\left(x_{2}, t_{2}\right) \neq 0
$$

This recovers the classical connectivity relation $(\mathrm{C} 1)$ If $\mathrm{aCb}$, then $\mathrm{a}>0$ and $\mathrm{b}>0$ :

$$
\Phi_{1}\left(x_{1}, t_{1}\right) \Phi_{2}\left(x_{2}, t_{2}\right) \neq 0 \rightarrow \Phi_{1}\left(x_{1}, t_{1}\right) \neq 0 \wedge \Phi_{2}\left(x_{2}, t_{2}\right) \neq 0
$$

Expressed in words this relation reads: Any two things which (i) have existed, (ii) will exist or (iii) currently are existing in the 3D universe are (4D) connected. They may however exist at different times i.e. they do "notCoexist" and thus are temporally disconnected. They also may coexist but may "notCollocate" i.e. they may be spatially disconnected. 


\section{1. isTimeConnected: "coexistence"}

"Coexistence" in a first place requires the two things to exist individually during some time intervals $t_{i}, t_{1}$ :

$$
\begin{aligned}
& \Phi_{k} \text { exists during } t_{i} \rightarrow \exists t_{i} \wedge \Phi_{k}\left(t_{i}\right) \neq 0 \\
& \Phi_{n} \text { exists during } t_{l} \rightarrow \exists t_{l} \wedge \Phi_{n}\left(t_{l}\right) \neq 0
\end{aligned}
$$

Coexistence can then be defined as both things existing during the same time interval to

$$
\Phi_{n} \text { coexists } \Phi_{k} \equiv \exists t_{0} \text { such that } \Phi_{k}\left(t_{0}\right) \neq 0 \wedge \Phi_{n}\left(t_{0}\right) \neq 0
$$

This is equivalent to a non-vanishing algebraic product describing the time-correlation $\Phi_{\mathrm{k}} \Phi_{\mathrm{n}}$ during that time interval to:

$$
\Phi_{n} \text { coexists } \Phi_{k} \rightarrow \Phi_{k}\left(t_{0}\right) \Phi_{n}\left(t_{0}\right) \neq 0
$$

Their temporal distance $\mathrm{dt}$ vanishes in this case because $\mathrm{t}_{\mathrm{i}}=\mathrm{t}_{1}\left(=\mathrm{t}_{0}\right)$ :

$$
d t \equiv t_{i}-t_{l}=0
$$

\section{2. isSpaceConnected: "collocation"}

The aspect of "collocation" has already been addressed in sections 3 and 4, where mereotopology and the time-independent phase field perspective were discussed in detail. In case of time dependent phenomena, 3D spatial connectivity is not static any more but becomes subject to change. The "isCollocated" relation thus needs to be complemented by a "wasCollocated" relation and in a similar way "coexists" needs a complements like "coexisted". Also the relation "isPhysicallyConnected" introduced in the following section needs such a complement relation "wasPhysicallyConnected". An instructive situation for such relations is depicted in Fig. 19. Such relations can easily be formally defined based on the scheme depicted in section 5.3. Although it would be possible to formally define relations for "willBeConnected" or similar relations directing to the future, this does not seem meaningful.

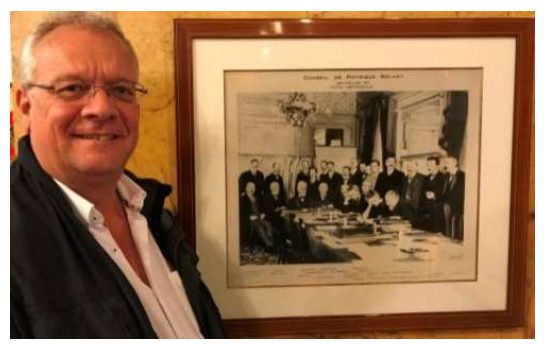

Fig. 19: Collocation and Coexistence:

Location: Hotel Metropol in Brussels, Belgium. I wasCollocated with the giants of physics at the time this "picture in picture" was taken. I was at the hotel and they had been at the same place in 1911. I "coexisted" only with two of the participants (M. deBroglie and G. Hostelet) which still were alive when I was born. At the time the

picture in picture was recorded, I wasPhysicallyConnected with the picture of the participants of the $1^{\text {st }}$ Solvay Conference. The original was recorded in 1911 and thus wasPhysicallyConnected to the participants. The participants werePhysicallyConnected as they wereCoexisting in 1911 AND wereCollocated at the Hotel Metropol during the conference period.

\section{3. isPhysically Connected}

Two things are physically connected in case they share a common region of space $\mathrm{x}_{0}$ during a finite time interval to in which both are coexisting. They are coexisting and collocated in this case:

$$
\Phi_{n} \text { isPhysicallyConnected } \Phi_{k} \equiv \Phi_{n} \text { isCollocated } \Phi_{k} \wedge \Phi_{n} \text { coexists } \Phi_{k}
$$

Their phase-field description is a function of both variables $\mathrm{x}$ and $\mathrm{t}$ in this case. 


$$
\begin{gathered}
\Phi_{n} \text { isPhysicallyConnected } \Phi_{k} \\
\rightarrow \exists x_{0}, t_{0} \text { such that } \Phi_{k}\left(x_{0}, t_{0}\right) \neq 0 \wedge \Phi_{n}\left(x_{0}, t_{0}\right) \neq 0
\end{gathered}
$$

In a simple next step even a relation "wasPhysicallyConnected" can be defined for things which have formerly coexisted AND were collocated during a time interval $t_{\text {past. }}$ They shared some former region $x$ (remember $x$ to be a finite region) during some past time interval $t_{\text {past }}(t$ is also finite). „Now" (i.e. during a time interval tnow) they arePhysicallyDisConnected:

$$
\begin{gathered}
\Phi_{n} \text { isPhysicallyDisConnected } \Phi_{k} \\
\rightarrow \nexists x_{0}, \exists t_{\text {now }} \text { such that } \Phi_{k}\left(x_{0}, t_{\text {now }}\right) \neq 0 \wedge \Phi_{n}\left(x_{0}, t_{\text {now }}\right) \neq 0
\end{gathered}
$$

\section{4. is CausallyConnected}

In case two things are both spatially AND temporally disconnected - i.e. both their spatial AND their temporal distance do not vanish - they still may be connected by a relation isCausally Connected. The situation is summarized in Fig. 20:

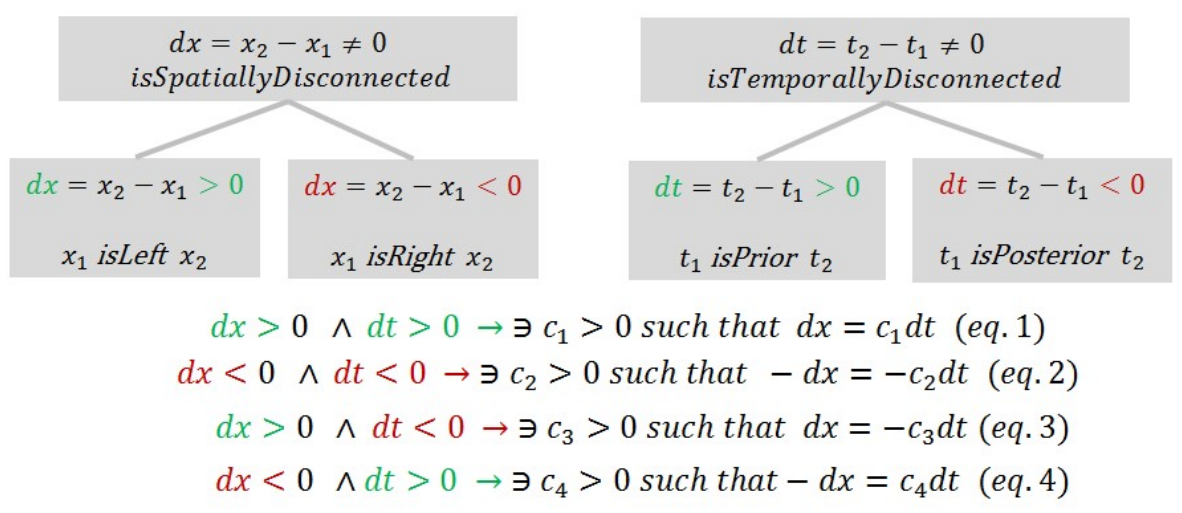

Fig. 20: The $\mathrm{C}_{\mathrm{i}}$ in the different equations a priori are not necessary identical. Requesting invariance under parity operations $(P(x)=-x)$ and under time inversal symmetry operations $(\mathrm{T}(\mathrm{t})=-\mathrm{t})$ however limits their choice. Applying the parity operation on eq. 1 leads to eq. 4 if $\mathrm{c}_{1}=\mathrm{C}_{4}$, applying it to eq. 2 leads to eq. 3 if $\mathrm{c}_{2}=\mathrm{C}_{3}$. Applying the time inversal operation to eq. 2 leads to eq. 4 if $\mathrm{c}_{2}=\mathrm{C}_{4}$. Eventually applying it to eq. 3 leads to eq. 1 if $\mathrm{C}_{3}=\mathrm{C} 1$. The request for satisfying invariance under both of these operations thus leads to $\mathrm{C}_{1}=\mathrm{C}_{2}=\mathrm{C}_{3}=\mathrm{C}_{4}=\mathrm{c}$.

In case equations 1 and 2 in Fig. 20 are identical (i.e. $\left.\mathrm{c}_{1}=\mathrm{C}_{2}\right)$ and they read:

$$
c d t-d x=0
$$

Equations 3 and 4 in Fig. 20 are also identical if $\mathrm{c}_{3}=\mathrm{C}_{4}$ and then read

$$
c d t+d x=0
$$

At least one of these equations is always satisfied as the original 4 equations cover all possible combinations. Following expression accordingly is always "true":

$$
c d t-d x=0 \quad \vee c d t+d x=0
$$

These conditions together correspond to the solution of a quadratic equation and accordingly can be re-formulated and combined into a single equation: 


$$
(c d t-d x)(c d t+d x)=0
$$

This equation specifies the invariant spacetime distance $\mathrm{ds}^{2}$ known from special relativity theory to be equal to 0 as a criterion for things being causally (i.e. light-like) connected:

$$
c^{2} d t^{2}-d x^{2}=0=d s^{2}
$$

\section{5. isEnergeticallyConnected}

As an observation, there is a striking formal similarity between the invariant spacetime interval and the energy-momentum balance of massless particles:

$$
\begin{gathered}
c^{2} \mathrm{dt}^{2}-d x^{2}=0 \\
E^{2}-p^{2} c^{2}=0
\end{gathered}
$$

Multiplying the first equation with a factor $\mathrm{F}^{2}$ - which a priori shall only represent a conversion factor between spatial coordinates (first equation) and energetic perspective (second equation) - yields

$$
\mathrm{F}^{2} \mathrm{c}^{2} \mathrm{dt}^{2}-\mathrm{F}^{2} \mathrm{dx}^{2}=0
$$

Recovering the following expressions - actually the definitions - for energy $\mathrm{E}$ and momentum $\mathrm{p}$ - one immediately gets the second equation

$$
\mathrm{dE}=\mathrm{Fdx} \text { and } \mathrm{dp}=\mathrm{Fdt} \rightarrow \mathrm{dp}^{2} \mathrm{c}^{2}-\mathrm{dE}^{2}=0
$$

Much more interesting, however, seems the definition of a relation isEnergeticallyConnected which means "equilibrium". Any distance from equilibrium then corresponds to a thermodynamic driving force e.g. for phase transitions. Such a mereotopological view on thermodynamic equilibrium has quite recently been discussed [79].

\section{6. isPathConnected}

Regions where the thing $\Phi_{i}$ and an elementary region of the reference frame $x_{j}$ collocate can be described by a non-vanishing correlation (see section 4 and Appendix B):

$$
\Phi_{i} x_{j} \neq 0
$$

Such a non-vanishing correlation holds for both of the following cases (see also Figure B4 in Appendix B):

$$
x_{j} \text { isProperPart } \Phi_{i} \quad \text { OR } \quad \Phi_{i} \text { isProperPart } x_{j}
$$

In case $\Phi$ isProperPart of $x_{j}$ the correlation denotes the position of this thing $\Phi$, as the correlations of $\Phi$ with all other VolumeElements $\mathrm{x}_{\mathrm{k}}$ are identical 0 in this case. This leads to:

$$
\sum_{k=1}^{N x} \Phi_{i} x_{k}=\Phi_{i} x_{j} \equiv x_{j}^{\Phi}
$$

This position may change over time. The time-dependent position is given by the coexistence of a time interval $\mathrm{t}_{\mathrm{j}}$, the thing $\Phi$ and the VolumeElement $\mathrm{x}_{\mathrm{k}}$ :

$$
\Phi_{i} x_{j} t_{k} \neq 0
$$

The position of a thing during a time interval $t_{k}$ (read "at time $t_{k}$ ") then can be written as:

$$
x_{j}^{\Phi}\left(t_{k}\right) \equiv x_{j}^{\Phi} t_{k}
$$


Characteristic points of any path are its initial and final positions (the "start-point" and the "end-point") denoting the two boundaries of a 4 dimensional object being small in 2 spatial dimensions (a "line" - see Appendix A) - the "path":

$$
\begin{aligned}
& \text { InitialPosition: } \quad x_{\text {initial }}^{\Phi} \equiv x_{0}^{\Phi}\left(t_{0}\right) \\
& \text { FinalPosition: } \\
& \mathrm{x}_{\mathrm{final}}^{\Phi} \equiv \mathrm{x}_{\mathrm{N}}^{\Phi}\left(\mathrm{t}_{\mathrm{N}}\right)
\end{aligned}
$$

An extended path then is a sequence of such positions bound by the initial and final positions which all exist during a sequence of different time intervals tk. The full path thus exists if any individual position has a non-zero value. For the extended path this means that the product of all these positions is non-zero for a sequence of time intervals:

$$
\text { A path from } x_{\text {initial }}^{\Phi} \text { to } \mathrm{x}_{\text {final }}^{\Phi} \text { exists } \rightarrow \prod_{k=0}^{N} x_{k}^{\Phi}\left(t_{k}\right) \neq 0
$$

The total "path" of a - tiny point like (i.e. smaller than the volume element $\mathrm{x}_{\mathrm{k}}$ ) - thing between two positions $x_{\text {initial }}^{\Phi}$ and $\mathrm{x}_{\text {final }}^{\Phi}$ taken by the thing at two different times 0 and $\mathrm{N}$ then can be summed up and yields the total volume $\mathrm{P}$ of the path "line":

$$
P^{\Phi}\left(x_{0}, x_{N}\right)=\sum_{k=0}^{N} x_{k}^{\Phi}\left(t_{k}\right)
$$

Any position $x_{k}^{\Phi}$ is a fraction of the whole and thus has a value smaller than 1 . The probability of a path to exist (which is given by the above product) thus decreases with increasing number $\mathrm{N}$ of path elements forming the product. This fact leads to a higher probability for shorter paths with the minimum path length being the most probable path. This is most likely related to the principle of least action and Fermat's principle. The sum and the product expressions find continuum counterparts in the Feynman path integrals ${ }^{18}$, with a rigorous formal comparison being subject to future work. The interesting fact here seems, that these expressions are derived based on mere logic considerations.

\section{Summary, Conclusions and Outlook}

The present article has related and compared the concepts of mereotopology, Region Connect Calculus and Contact Algebra to a field theoretic description of objects, their shapes and their boundaries by the phase-field concept. The concepts underlying mereotopology on the one hand seemed to be similar to the principles underlying the phase-field concept, on the other hand a number of differences/complementarities became obvious.

In a first place limitations of Boolean algebra were identified. Boolean algebra does not allow for the coexistence of a thing and its complement. Such coexistence, however, is essential not only in the phase-field perspective, where coexistence and collocation actually are measures for the physical boundary between things. Mereotopology is based on Boolean algebra and thus considers the different types of connectivity as logically -and thus qualitatively - disjoint. Transitions e.g. from " $x$ isDisconnected $y$ " to " $x$ isProperPartof $y^{\prime \prime}$ - with a cherry dropping into whipped cream being an example - thus cannot or only hardly be addressed. Transitions, however, are ubiquitous processes and thus need a formal description. Such a description may widen the field of applications of contemporary mereology resp. mereotopology and mereogeometry towards new grounds of mereophysics.

A "dynamic" view on contacts and boundaries and the notion of time were thus introduced. This is first reflected in time dependent relations allowing to semantically de-

18 https://en.wikipedia.org/wiki/Path_integral_formulation 
scribe "historicalParthood" via "isConnected" or "wasConnected" relations. Separating the description of 4D connectivity into "isSpatiallyConnected" and "isTemporallyConnected" allowed for the definition of isPhysciallyConnected and eventually also of isCausallyConnected. As a consequence, the formulation of the relativistic spacetime interval could be derived from mere logical inference.

Eventually the symmetry of the connectivity relation was discussed, where a isPathConnected and a isEnergeticallyConnected relation provide the grounds for describing reversible and/or irreversible paths.

In a generalized notion the "isConnected" relation according to the present article might be interpreted as some generalized "distance" between two things to be equal to 0 . This generalized distance may be a spatial distance, a temporal distance, an energetic distance or any other type of general distance. All these distances are expressed as the difference between two values. The status of "isConnected" thus is reached if two values are equal, i.e. if two values satisfy the equation of type $A-B=0$. A possible notion of "distance" was introduced, which could be described in mereological terms as minimal self-connected underlap minus mereological sum of two disconnected things.

Concepts of higher order junctions were discussed. These relate to connectivity of more than two things. Benefits of describing triple junctions with triadic relations and the importance of triadic relations in general were highlighted as well as the notion of helicity and aspects of 3D connectivity of triple junctions being seemingly disconnected in 2D. A potential need to reconsider/reformulate one of the axioms in Contact Algebra was identified when discussing connectivity of multiple objects.

Author Contributions: The entire article has been conceptualized and written by the author GJS.

Funding: The work presented in this article is a spin-off from discussions during the formulation of the Elementary Multiperspective Material Ontology EMMO being funded by the European Commission in the MarketPlace project under grant \# 760173 and from discussions on applications of EMMO for Metamodels and Digital Shadows within the WorkStream AII of the Cluster of Excellence "Internet of Production" funded by the Deutsche Forschungsgemeinschaft DFG under grant [EXC 2023, Project-ID: 39062112].

Acknowledgments: The author thanks Emanuele Ghedini, Jesper Friis, Gerhard Goldbeck, Adham Hashibon and Sebastiano Moruzzi for their patience and for all the interesting and constructive discussions on philosophy and on various areas of physics during the intense work on EMMO.

Conflicts of Interest: The author declares no conflict of interest. The funders had no role in the design of the study; in the collection, analyses, or interpretation of data; in the writing of the manuscript, or in the decision to publish the results. 


\section{Appendix A: 4D Geometry}

This appendix proposes a solution to the dilemma of describing points, lines, surfaces, and volumes known from classical geometry - and all having different dimensions there - all as $4 \mathrm{D}$ physicals. Based on a recent essay to derive physics laws from mereology [1] and the mereological principle that any part of a $4 \mathrm{D}$ item again has to be a $4 \mathrm{D}$ item, the basic approach to a solution is shortly outlined in the following in a very simplified way.

Any volume in $3 \mathrm{D}_{3 \mathrm{D}}$ is the product of a length $\mathrm{L}$, a width $\mathrm{W}$, and a height $\mathrm{H}$ and can be written as scalar triple product:

$$
H \overrightarrow{\mathrm{e}}_{\mathrm{z}}\left(L \overrightarrow{\mathrm{e}}_{\mathrm{x}} \times W \overrightarrow{\mathrm{e}}_{\mathrm{y}}\right)=\mathrm{V}_{3 D}
$$

Assuming further the length $\mathrm{L}$ (and width $\mathrm{W}$ and height $\mathrm{H}$ ) being - integer - multiples of an elementary length scale $l_{p}$ allows rewriting

$$
\begin{aligned}
& h \overrightarrow{\mathrm{e}}_{\mathrm{z}}\left(l \overrightarrow{\mathrm{e}}_{\mathrm{x}} \times w \overrightarrow{\mathrm{e}}_{\mathrm{y}}\right) * \mathrm{l}_{p}^{3}=\mathrm{V}_{3 D} \\
& \text { with integers } h, l, w \geq 1
\end{aligned}
$$

Volumes being thin in one of the three spatial dimensions - i.e. surfaces - then are defined as $3 \mathrm{D}$ volumes where exactly one of the integers takes the value 1 . Lines correspond to 3D volumes where exactly 2 of these integers take the value 1 and points are elementary volumes in which all 3 integers take the value 1 .

The time $\mathrm{T}$ as $4^{\text {th }}$ dimension becomes a further factor in the product defining the volume in $4 \mathrm{D}\left(\mathrm{V}_{4 \mathrm{D}}\right) . c T$ is an integer multiple of a length scale $l p=c \tau p$

$$
\begin{gathered}
V_{4 D}=V_{3 D} c T=V_{3 D} t c \tau_{p} \\
\text { with integer } t \geq 1
\end{gathered}
$$

All objects in following scheme, figure A1, accordingly are 4D spacetime items:

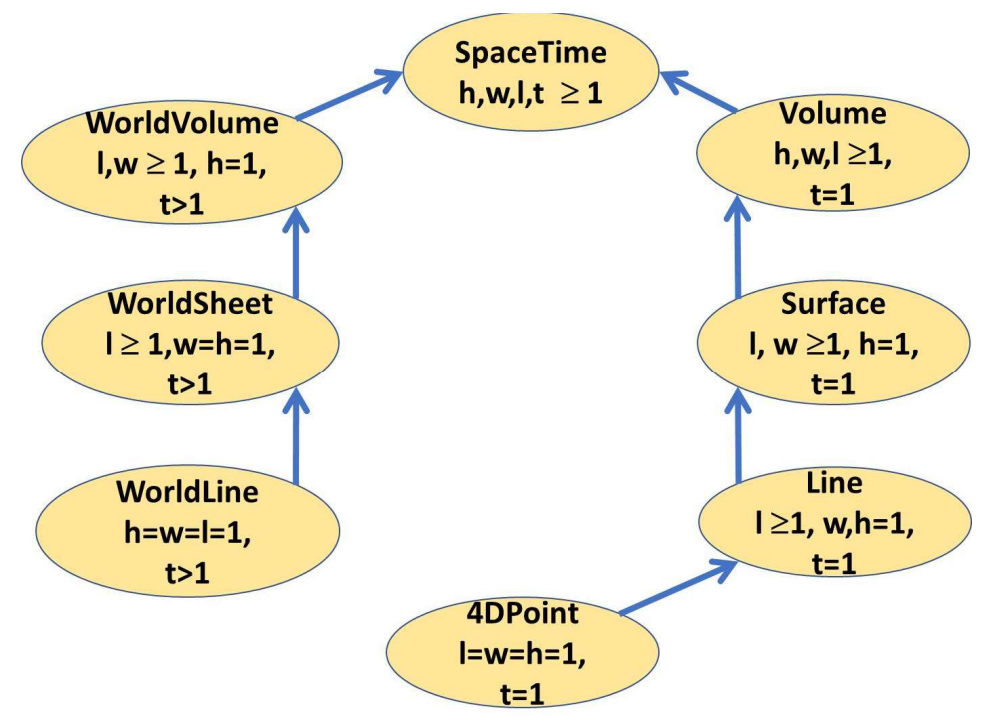

Figure A1: Concept to integrate different 4D objects ("points", "lines" "surfaces") as subclasses of a $4 \mathrm{D}$ physical spacetime. The classes are differentiated by the values of the variables $\mathrm{l}, \mathrm{w}, \mathrm{h}$, and $\mathrm{t}$ (see text). Arrows are to be read as "hasPart" relations. 


\section{Appendix B: From Mereology to a Scalar Field}

\section{B1 Reference frame}

To obtain information about the local existence or non-existence of things, their mutual positions and their topological arrangement in a region of spacetime, it is beneficial to discretize this region into smaller sub-regions specifying a "reference frame" or a "coordinate system".

\section{B2 Discretization of spacetime}

Multiplying the "basic equation" (which is based on the top axiom of mereology [1]) with a pseudoscalar entity like e.g. a volume $\mathrm{V}$ is the first step towards discretizing space:

$$
\sum_{i=0}^{N_{\Phi}} \Phi_{i} V=\sum_{i=0}^{N_{\Phi}} V_{i}=V
$$

$\mathrm{V}$ represents the total volume of the universe under consideration. Note that this volume and all the sub-regions $V_{i}$ are pseudoscalars (Figure B1 a). In a next step tiny elementary - cubes all having the same size - may be considered as abstracted regions. These do not need to be interconnected but might be embedded in an unstructured matrix (Figure B1 b) and/or B2 c))

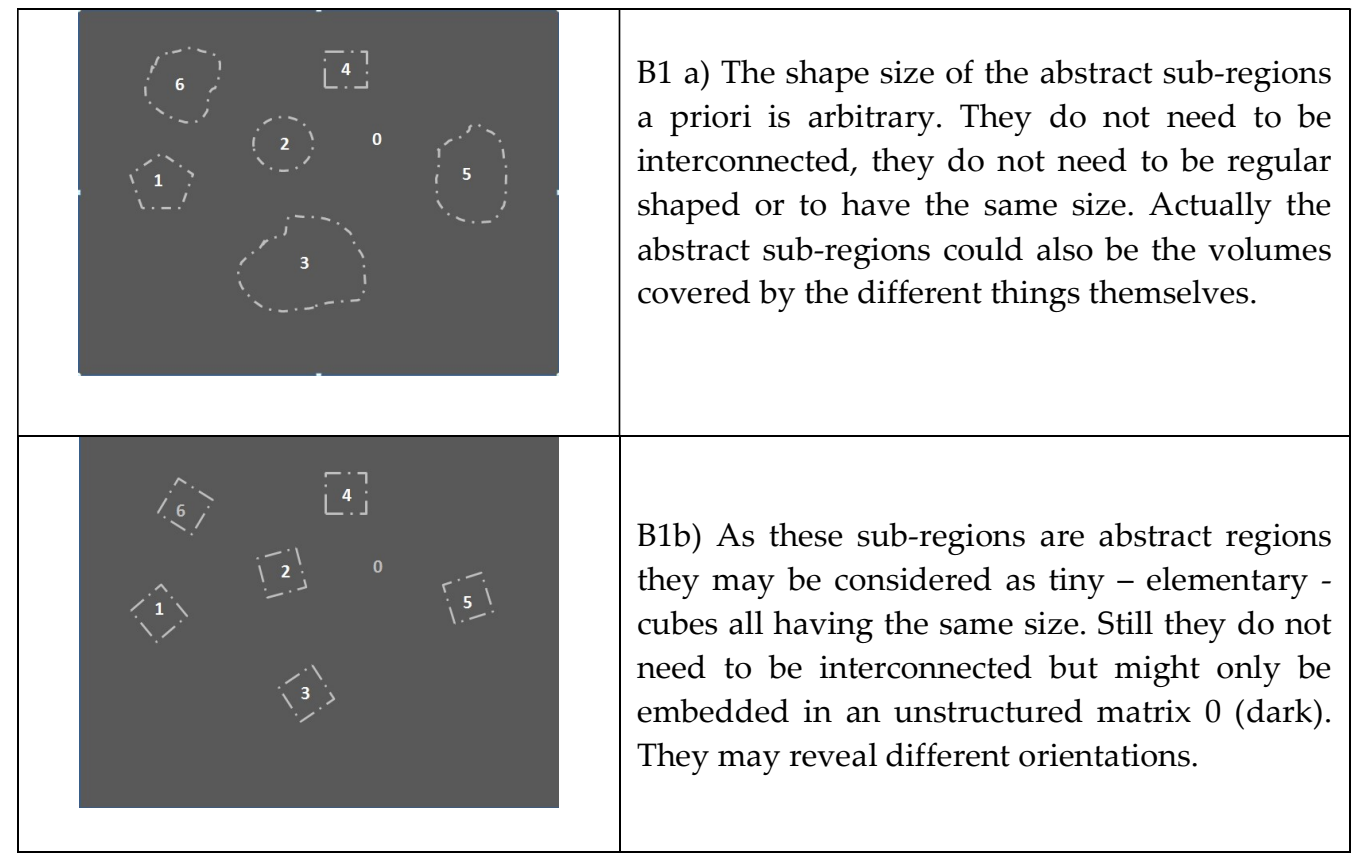

\section{B3 "Self-assembly" of spacetime}

In a further step the cubes can be arranged - or may by some process self-assemble into a regular lattice. This process might be interpreted as a condensation of a structured volume from an unstructured gas phase containing individual volumes. In an intermediate step molecule type arrangements of volume elements may form, Fig. B2 a: 


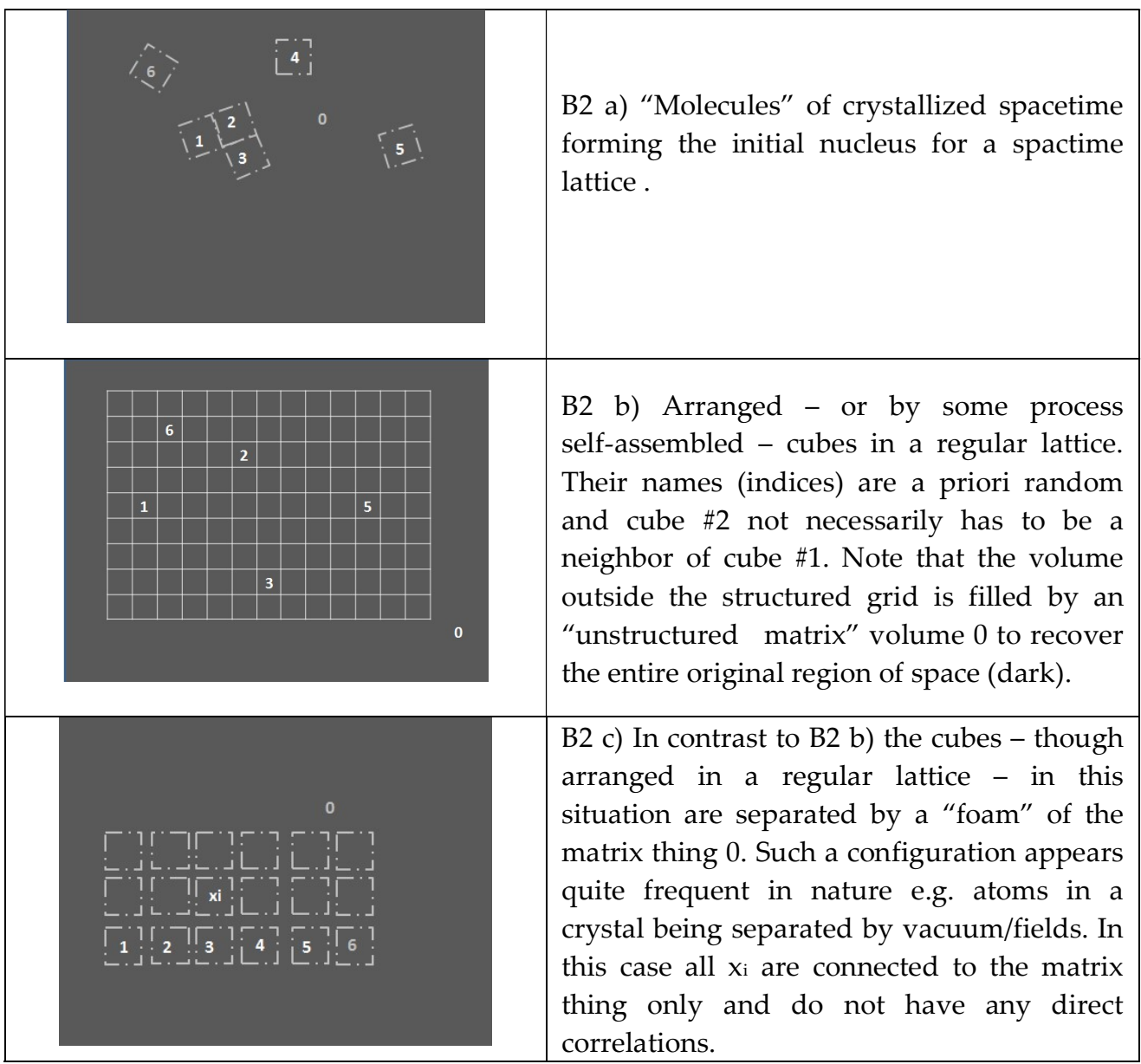

The number $\mathrm{N}$ of things in figure $\mathrm{B} 2$ is the total number of cubes, which for a - cube/ brick type- reference frame is $\mathrm{N}_{\mathrm{x}} \mathrm{N}_{\mathrm{y}} \mathrm{N}_{\mathrm{z}}$, see figure B3. Actually the region under consideration here is composed of a structured grid and an unstructured matrix $\mathrm{V}_{0}$

All cube volumes $V_{\text {i }}$ for $\mathrm{i}>0$ are identical with respect to their value and only differ with respect to their position in the lattice being denoted by their name/index $\mathrm{j}$. $\mathrm{V}_{0}$ fills all possible empty space between the cubes and also the remaining parts of the volume of the universe not being filled by the cubes.

$$
V_{0}+\sum_{j=1}^{N_{x} N_{y} N_{z}} V_{j}=V
$$

In case nothing exists in the Volume 0, everything exists in the structured volume. The total volume than can be re-sized to the structured part $\mathrm{V}^{\prime}$ only

$$
\sum_{j=1}^{N_{x} N_{y} N_{z}} V_{j}=V^{\prime}
$$

Speculative side remark: Comparing this "self-assembly" process with a crystallization process further suggests the possible release of some latent heat, which might be the initial energy being present during the big bang (which would be a big nucleation and crystallization process and not an explosion in this picture). 


\section{B4 Neighborhood relations}

The names/indices of the individual volume elements in the preceding section were selected arbitrary. A simple renaming does not affect the volume elements except changing their name. By convention, naming the indices of the cubes allows for the introduction of neighborhoods e.g. cube \# 5 is left neighbor of cube \#6 and right neighbor of cube\#4 in a linear, one dimensional chain of cubes. This naming scheme can easily be extended also to 3D, Figure B3.

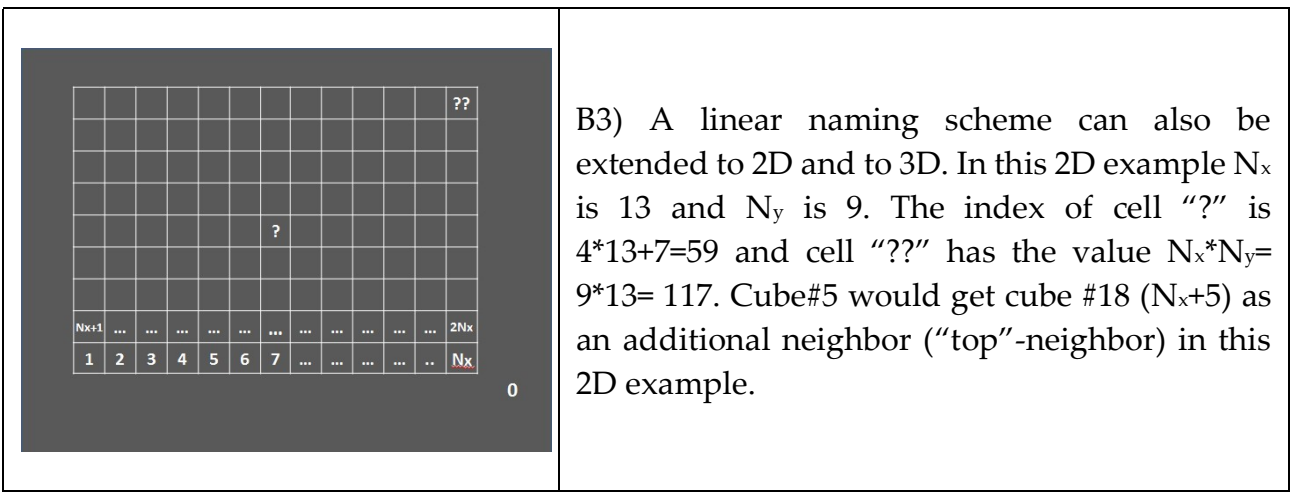

This naming step is most important as it introduces and defines "left" and "right" (in one dimension) and also front/rear and top/bottom in 3 dimensions. Such neighborhood relations are essential and open up the possibility to describe relative positions of things.

\section{B5 Positions and Scalar Fields}

For a $3 \mathrm{D}$ situation the number of the cubes is $\mathrm{N}_{\mathrm{x}} \mathrm{N}_{\mathrm{y}} \mathrm{N}_{\mathrm{z}}$. The index has been switched to $j$ here to distinguish it from the index $i$ being used to count the things $\Phi_{\mathrm{i}}$. The volume of the structured spacetime $\mathrm{V}^{\prime}$ is considered only in the following.

$$
\begin{gathered}
\sum_{j=1}^{N_{x} N_{y} N_{z}} \frac{V_{j}}{V^{\prime}}=1 \\
\sum_{j=1}^{N_{x} N_{y} N_{z}} \frac{V_{j}}{V^{\prime}}=1
\end{gathered}
$$

This equation can be directly multiplied with the basic equation for the -scalarthings $\Phi_{\mathrm{i}}$ yielding

$$
\begin{aligned}
& \sum_{\substack{j=1 \\
N_{x} N_{y} N_{z}}}^{N_{x} N_{y} N_{z}} \frac{V_{j}}{V^{\prime}} \sum_{i=0}^{N_{\Phi}} \Phi_{i}=1 \\
& \sum_{j=1}^{N_{\phi}} \sum_{i=0}^{V_{j}} V_{j} \Phi_{i}=V^{\prime}
\end{aligned}
$$

For a single thing in vacuum/matrix $\left(N_{\Phi}=1\right)$ being represented on this "grid" this equation reads

$$
\sum_{j=1}^{N_{x} N_{y} N_{z}} \sum_{i=0}^{1} V_{j} \Phi_{i}=V^{\prime}
$$


The term

$$
\sum_{j=1}^{N_{x} N_{y} N_{z}}\left(V_{j} \Phi_{1}+V_{j} \Phi_{0}\right)=V^{\prime}
$$

$$
\mathrm{V}_{\mathrm{j}} \Phi_{1} \equiv \Phi_{1}\left(\mathrm{~V}_{\mathrm{j}}\right)
$$

is the correlation between the elementary cube volume $V_{j}$ and the thing 1 . This product only exists where both $\mathrm{V}_{\mathrm{j}}$ and $\Phi$ coexist i.e. where both have non zero values.

The value of the index $j$ corresponds to a well-defined position $r_{j}$ of the centroid of the cube $\mathrm{j}$ as defined by the numbering/naming scheme being detailed above. The equation accordingly defines a discretized field describing the presence of thing 1 at various, discrete positions $\mathrm{r}_{\mathrm{j}}$ :

$$
\mathrm{V}_{\mathrm{j}} \Phi_{1} \equiv \Phi_{1}\left(\mathrm{~V}_{\mathrm{j}}\right)=\Phi_{1}\left(\overrightarrow{\mathrm{r}}_{\mathrm{j}}\right)
$$

In case the volume of the $\Phi_{1}$ is smaller than the volume element $V_{j}$, this correlation only exists inside this particular volume $V_{j}$ and the term then defines the position of the thing $\Phi_{\mathrm{i}}$

$$
\mathrm{V}_{\mathrm{j}} \Phi_{1}=\mathrm{V}_{\mathrm{j}}\left(\Phi_{1}\right)=\overrightarrow{\mathrm{r}}_{\mathrm{j}}\left(\Phi_{1}\right) \text { for } \quad \Phi_{1}<\mathrm{V}_{\mathrm{j}}
$$

These two cases are illustrated in Fig B4:
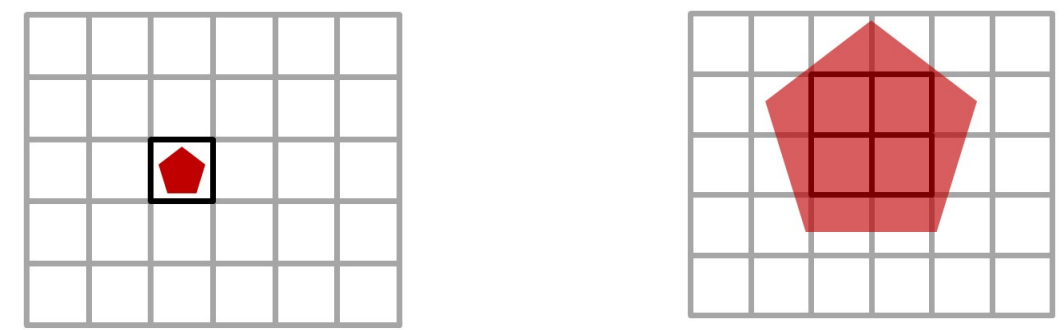

Figure B4: Left: In case $\Phi$ (red) isProperPart of a specific VolumeElement $x_{j}$ (depicted by the black boundary) this particular VolumeElement defines the position of $\Phi$ up to some remaining uncertainty. Right: All VolumeElements $x_{j}$ being parts of $\Phi$ denote regions where $\Phi$ is present. In the case they are ProperParts of $\Phi$, there is no other thing present in this particular VolumeElement.

In case the thing $\Phi_{1}$ is bigger than an individual $V_{j}$ the total volume of the thing $i$ then reads

$$
\sum_{j=0}^{N_{x} N_{y} N_{z}} V_{j} \Phi_{1}=V_{1}
$$

Remark: This equation in a continuum formulation corresponds to:

$$
\iiint_{0}^{\mathrm{V}} \Phi_{1}(\overrightarrow{\mathrm{r}}) \mathrm{dxdydz}=\mathrm{V}_{1}
$$

In a next step time intervals can be introduced by multiplying the basic equation with a scalar (not a pseudoscalar!) called time $\mathrm{T}$

$$
\begin{gathered}
\sum_{k=0}^{N_{t}} \Phi_{k} T=T \\
\sum_{k=0}^{N_{t}} t_{k}=T
\end{gathered}
$$


Similar to the selection of identical volumes, also identical sized time intervals can be selected making the index $\mathrm{k}$ a measure for the position of the respective time interval on the arrow of time. A Volume $\mathrm{j}$ exists during a time interval tk means:

$$
\mathrm{V}_{\mathrm{j}} \mathrm{t}_{\mathrm{k}}>1
$$

This is a $4 \mathrm{D}$ spacetime volume element. Thing i exists during a time interval $t_{k}$ means:

$$
\Phi_{\mathrm{i}} \mathrm{t}_{\mathrm{k}}>1
$$

"Coexisting" eventually means that the correlation also is a correlation with the time interval making the product not vanishing during that time interval

$$
\mathrm{V}_{\mathrm{j}} \Phi_{1} \mathrm{t}_{\mathrm{k}}>1
$$

Eventually, a scalar field description for a - discretized - scalar field describing the elementary regions covered by can be formulated

$$
\mathrm{V}_{\mathrm{j}} \Phi_{1} \mathrm{t}_{\mathrm{k}}=\Phi_{1}\left(\mathrm{~V}_{\mathrm{j}}, \mathrm{t}_{\mathrm{k}}\right)
$$

which in a continuous formulation (with $V_{j}$ and $t_{k}->0$ ) can be identified with a scalar field -the phase-field:

$$
\Phi_{1}\left(\vec{r}_{j}, t_{k}\right) \rightarrow \Phi_{1}(\vec{r}, t)
$$




\section{Appendix C: States and Interface States}

Quadruple junctions - i.e. the collocation of 4 things - correpond to "points" (which are still finite sized volumes). Looking at the fourth oder eponents of the basic eqaution thus can be expected to yield further insights. Raising the basic equation ot the fourth power

$$
1=\left(\Phi_{i}+\Phi_{j}+\Phi_{k}+\Phi_{l}\right)^{4}
$$

yields a total of $4^{4}=256$ terms which can be sorted using the multinomial expansion ${ }^{19}$ :

$$
\left(\Phi_{1}+\Phi_{2}+\Phi_{3}+\Phi_{4}\right)^{4}=\sum_{k 1+k 2+k 3+k 4=4} \frac{4 !}{k 1 ! k 2 ! k 3 ! k 4 !} \Phi_{1}^{k 1} \Phi_{2}^{k 2} \Phi_{3}^{k 3} \Phi_{4}^{k 4}
$$

The $\mathrm{k}_{\mathrm{i}}$ always sum up to 4 by definition. This allows classifying into

- 4 "unary" terms $\partial \Phi_{i}$ with one of the ki being equal to 4 and all others being identical 0

- 84 "dual" boundary terms, where two of the $\mathrm{k}_{\mathrm{i}}$ are identical 0 and the others complement to 4

- 144 "triple" boundary terms, where one of the $\mathrm{k}_{\mathrm{i}}$ is identical 0 and the three others complement to 4

- 24 "quadruple" boundary terms, where all $\mathrm{k}_{\mathrm{i}}$ are identical 1

Unary states:

One $\mathrm{k}_{\mathrm{i}}=4$ and the others equal to 0 yields a total of 4 states:

$$
\frac{4 !}{4 ! 0 ! 0 ! 0 !} \Phi_{1}^{4}, \quad \frac{4 !}{0 ! 4 ! 0 ! 0 !} \Phi_{2}^{4}, \quad \frac{4 !}{0 ! 0 ! 4 ! 0 !} \Phi_{3}^{4}, \quad \frac{4 !}{0 ! 0 ! 0 ! 4 !} \Phi_{4}^{4}
$$

Dual boundaries:

Two $\mathrm{k}_{\mathrm{i}}=0$ e.g. $\mathrm{k}_{3}=0 \wedge \mathrm{k}_{4}=0$.

(i) Two other $\mathrm{k}$ are identical and complement to 4 e.g. $\quad \mathrm{k}_{1}=\mathrm{k}_{2}=2$

$$
\frac{4 !}{2 ! 2 ! 0 ! 0 !}=6 * 6 \text { dual boundaries }=36
$$

(i) Two other $\mathrm{k}$ are different and complement to 4 e.g. $\mathrm{k}_{1}=1 \wedge \mathrm{k}_{2}=3$ or $\mathrm{k}_{1}=3 \wedge \mathrm{k}_{2}=1$

$$
\begin{aligned}
& \frac{4 !}{1 ! 3 ! 0 ! 0 !}=4 * 6 \text { dual boundaries }=24 \\
& \frac{4 !}{3 ! 1 ! 0 ! 0 !}=4 * 6 \text { dual boundaries }=24
\end{aligned}
$$

In total the description of a dual boundary $\mathrm{i}, \mathrm{k}$ thus comprises 3 types linear independant states (i.e. $\Phi_{i}^{2} \Phi_{k}^{2} ; \Phi_{i}^{3} \Phi_{k}^{1}$ and $\Phi_{i}^{1} \Phi_{k}^{3}$ ), which might be related to a "pseudo-vector" perpendicular to the boundary. A total of 6 dual boundaries exists: $\left(\mathrm{k}_{3}=0 \wedge \mathrm{k}_{4}=0 \mathrm{~V}\right.$ $\left.\mathrm{k}_{2}=0 \wedge \mathrm{k}_{4}=0 \vee \mathrm{k}_{1}=0 \wedge \mathrm{k}_{4}=0 \vee \mathrm{k}_{2}=0 \wedge \mathrm{k}_{3}=0 \vee \mathrm{k}_{2}=0 \wedge \mathrm{k}_{1}=0 \vee \mathrm{k}_{1}=0 \wedge \mathrm{k}_{3}=0\right)$

\section{Ternary junctions:}

One of the $k_{i}=0$ (e.g. $\left.k_{4}\right)$, two $k_{i}=1$ and one $k_{i}=2$.

There is a total of 4 Triple junctions (i.e. $\mathrm{k}_{1}=0 \vee \mathrm{k}_{2}=0 \vee \mathrm{k}_{3}=0 \vee \mathrm{k}_{4}=0$ ):

19 https://en.wikipedia.org/wiki/Multinomial_theorem 


$$
\begin{aligned}
& \frac{4 !}{1 ! 1 ! 2 ! 0 !}=12 * 4 \text { triple junctions }=48 \\
& \frac{4 !}{1 ! 2 ! 1 ! 0 !}=12 * 4 \text { triple juncttions }=48 \\
& \frac{4 !}{2 ! 1 ! 1 ! 0 !}=12 * 4 \text { triple juncttions }=48
\end{aligned}
$$

In total the description of a ternary junction $\mathrm{i}, \mathrm{j}, \mathrm{k}$ (a "triple boundary") thus also comprises 3 linear independent states (i.e. $\Phi_{i}^{1} \Phi_{j}^{1} \Phi_{k}^{2} ; \Phi_{i}^{1} \Phi_{j}^{2} \Phi_{k}^{1}$ and $\Phi_{i}^{2} \Phi_{j}^{1} \Phi_{k}^{1}$ ), which might be related to a "vector" parallel to the triple boundary line.

Quaternary Junction

All $\mathrm{k}_{\mathrm{i}}$ are equal: $\left(\mathrm{k}_{1}=\mathrm{k}_{2}=\mathrm{k}_{3}=\mathrm{k}_{4}=1\right)$ :

$$
\frac{4 !}{1 ! 1 ! 1 ! 1 !}=24
$$

Some simplications of above expressions are possible. The general unary term for object i:

simplifies to

$$
\partial \Phi_{i}=\sum_{\substack{k_{i}=4 \\ k_{j}=k_{k}=k_{l}=0}} \frac{4 !}{k_{i} ! k_{j} ! k_{k} ! k_{l} !} \Phi_{i}^{k_{i}} \Phi_{j}^{k_{j}} \Phi_{k}^{k_{k}} \Phi_{l}^{k_{l}}
$$

$$
\partial \Phi_{i}=\Phi_{i}^{4}
$$

The Dual boundary terms for a single, boundary between $\mathrm{i}$ and $\mathrm{j}$

$$
\partial \Phi_{i, j}=\sum_{\substack{k_{i}+k_{j}=4 \\ k_{k}=k_{l}=0}} \frac{4 !}{k_{i} ! k_{j} ! k_{k} ! k_{l} !} \Phi_{i}^{k_{i}} \Phi_{j}^{k_{j}} \Phi_{k}^{k_{k}} \Phi_{l}^{k_{l}}
$$

simplify to

and further to

$$
\partial \Phi_{i, j}=\sum_{k_{i}+k_{j}=4} \frac{4 !}{k_{i} ! k_{j} ! 0 ! 0 !} \Phi_{i}^{k_{i}} \Phi_{j}^{k_{j}} \Phi_{k}^{0} \Phi_{l}^{0}
$$

This term then simplifies and splits into

$$
\partial \Phi_{i, j}=\sum_{k_{i}+k_{j}=4} \frac{4 !}{k_{i} ! k_{j} !} \Phi_{i}^{k_{i}} \Phi_{j}^{k_{j}}
$$

$$
\partial \Phi_{i, j}=\sum_{k_{i}=k_{j}=2} \frac{4 !}{2 ! 2 !} \Phi_{i}^{2} \Phi_{j}^{2}=6 \Phi_{i}^{2} \Phi_{j}^{2}
$$

plus

$$
\sum_{k_{i}=3 \wedge k_{j}=1} \frac{4 !}{3 ! 1 !} \Phi_{i}^{3} \Phi_{j}^{1}=4 \Phi_{i}^{3} \Phi_{j}^{1}
$$

plus

$$
\sum_{k_{i}=1 \wedge k_{j}=3} \frac{4 !}{1 ! 3 !} \Phi_{i}^{3} \Phi_{j}^{1}=4 \Phi_{i}^{1} \Phi_{j}^{3}
$$

The triple junction terms for junctions involving $\mathrm{i}, \mathrm{j}$ and $\mathrm{k}(\mathrm{i} . \mathrm{e} \mathrm{kl}=0$ ) 
simplify to

$$
\partial \Phi_{i, j, k}=\sum_{\substack{k_{i}+k_{j}+k_{k}=4 \\ k_{l}=0}} \frac{4 !}{k_{i} ! k_{j} ! k_{k} ! k_{l} !} \Phi_{i}^{k_{i}} \Phi_{j}^{k_{j}} \Phi_{k}^{k_{k}} \Phi_{l}^{k_{l}}
$$

and can be split into

$$
\partial \Phi_{i, j, k}=\sum_{k_{i}+k_{j}+k_{k}=4} \frac{4 !}{k_{i} ! k_{j} ! k_{k} !} \Phi_{i}^{k_{i}} \Phi_{j}^{k_{j}} \Phi_{k}^{k_{k}}
$$

$$
\partial \Phi_{i, j, k}=\sum_{\substack{k_{i}=2 \\ k_{j}=k_{k}=1}} \frac{4 !}{2 ! 1 ! 1 !} \Phi_{i}^{2} \Phi_{j}^{1} \Phi_{k}^{1}=12 \Phi_{i}^{2} \Phi_{j}^{1} \Phi_{k}^{1}
$$

plus

$$
\sum_{\substack{k_{j}=2 \\ k_{i}=k_{k}=1}} \frac{4 !}{1 ! 2 ! 1 !} \Phi_{i}^{1} \Phi_{j}^{2} \Phi_{k}^{1}=12 \Phi_{i}^{1} \Phi_{j}^{2} \Phi_{k}^{1}
$$

plus

$$
\sum_{\substack{k_{k}=2 \\ k_{i}=k_{j}=1}} \frac{4 !}{1 ! 1 ! 2 !} \Phi_{i}^{1} \Phi_{j}^{1} \Phi_{k}^{2}=12 \Phi_{i}^{1} \Phi_{j}^{1} \Phi_{k}^{2}
$$

These triple junction terms eventually sum in total to

$$
\partial \Phi_{i, j, k}=12 \Phi_{i}^{2} \Phi_{j}^{1} \Phi_{k}^{1}+12 \Phi_{i}^{1} \Phi_{j}^{2} \Phi_{k}^{1}+12 \Phi_{i}^{1} \Phi_{j}^{1} \Phi_{k}^{2}
$$

Which can be split into cylic and anticyclic permutations

$$
\begin{aligned}
\partial \Phi_{i, j, k}=\partial^{+} \Phi_{i, j, k} & +\partial^{+} \Phi_{i, k, j} \\
& =\left(6 \Phi_{i}^{2} \Phi_{j}^{1} \Phi_{k}^{1}+6 \Phi_{i}^{1} \Phi_{j}^{2} \Phi_{k}^{1}+6 \Phi_{i}^{1} \Phi_{j}^{1} \Phi_{k}^{2}\right) \\
& +\left(6 \Phi_{i}^{2} \Phi_{k}^{1} \Phi_{j}^{1}+6 \Phi_{i}^{1} \Phi_{k}^{2} \Phi_{j}^{1}+6 \Phi_{i}^{1} \Phi_{k}^{1} \Phi_{j}^{2}\right)
\end{aligned}
$$

The " 6 " ahead of each of the terms correspond to the number of cyclic permutations of the $\mathrm{i}, \mathrm{j}, \mathrm{k}$. This can be rewritten by introducing the two helicities (see section 3.4) into

using following definition

$$
\partial^{+} \Phi_{i, j, k}+\partial^{-} \Phi_{i, j, k}=\partial \Phi_{i, j, k}
$$

$$
\partial^{-} \Phi_{i, j, k}=\partial^{+} \Phi_{i, k, j}
$$




\section{Appendix D: Construction of an item from mereotopological / phase field information}

Scope of this appendix is to provide the solution to the challenge posed in section 4.3.6 and to discuss further options for a refined description of a geometrical state. The information provided for the solution of the challenge is listed again in following table. The aim was to describe the well-known Tai-Chi symbol (a) based on connectivity. A description of connectivity is necessary but not sufficient to describe this symbol. Symbol (b) would have the same topological description. The phase field concept brings further information about the relative sizes of the objects. The fraction object 1 takes of the universe shall be equal the fraction object 2 takes and the same holds for objects $3 \& 4)\left(\Phi_{1}=\right.$ $\Phi_{2} \wedge \Phi_{3}=\Phi_{4}$ ) Also the relative fractions of interfaces may be used as criteria. Note that the equal sign indicates the fractions the object take of the universe to be identical, but not the objects themselves. This further constrains the possible choices of a geometrical configuration matching all conditions but still does not lead to the Tai-Chi Symbol (c). Further constraints can be placed on the ratio of the surface (with finite thickness) to the area (remember that in mereology both have the same dimension) leading to a description of disc type objects (d). This brings the description of the Tai Chi symbol closer to the desired object. Still many things like "distances" or "orientations" are missing. Figuring out possible descriptions for further constraints is subject to future efforts.

\begin{tabular}{|c|c|}
\hline & $\begin{array}{l}\text { a): gray, } 1 \text { white, } 2 \text { black, } 3 \text { white, } 4 \text { black } \\
\text { Topological information (phase-field perspective): } \\
\qquad \begin{aligned} \partial \Phi_{1, \forall}= & \partial \Phi_{1,0}+\partial \Phi_{1,2}+\partial \Phi_{1,4}+\partial \Phi_{1,2,0}+\partial \Phi_{1,0,2} \\
\partial \Phi_{2, \forall}= & \partial \Phi_{2,0}+\partial \Phi_{2,1}+\partial \Phi_{2,3}+\partial \Phi_{2,1,0}+\partial \Phi_{2,0,1} \\
& \partial \Phi_{3, \forall}=\partial \Phi_{3,2} \quad \partial \Phi_{4, \forall}=\partial \Phi_{4,1}\end{aligned} \\
\begin{aligned} \partial \Phi_{0, \forall}= & \partial \Phi_{0,1}+\partial \Phi_{0,2}+\partial \Phi_{0,1,2}+\partial \Phi_{0,2,1}+\text { ext. }\end{aligned}\end{array}$ \\
\hline 0 & $\begin{array}{l}\text { b) Topological information (RCC perspective): } \\
1 \text { isConnected } 2 \text { is abbreviated as } 1 C 2 \\
\qquad C 0 \wedge 1 C 2 \wedge 1 C 4 \\
2 C 0 \wedge 2 C 1 \wedge 2 C 3 \\
3 C 2 \text { only, } 4 C 1 \text { only } \\
0 C 1 \wedge 0 C 2 \wedge \neg 0 C 3 \wedge \neg 0 C 4\end{array}$ \\
\hline & $\begin{array}{c}\text { c) "Relative quantitative information" (phase-field perspective): } \\
\Phi_{1}=\Phi_{2} \wedge \Phi_{3}=\Phi_{4} \\
\partial \Phi_{1,0}=\partial \Phi_{2,0} \wedge \partial \Phi_{1,4}=\partial \Phi_{2,3} \\
\partial \Phi_{1,0}>\partial \Phi_{1,4} \ldots \text { etc } \ldots\end{array}$ \\
\hline & $\begin{array}{l}\text { d) "further possible constraints": Requesting the ratio of "object } \\
\text { boundary"* to "object area" to be minimum: } \\
\qquad \frac{\partial \Phi_{3, \forall}}{\Phi_{3}}=! \text { min and } \frac{\partial \Phi_{4, \forall}}{\Phi_{4}}=! \mathrm{min} \\
\text { will constrain the two objects } 3 \text { and } 4 \text { to be discs. The same holds } \\
\text { for the total boundary of the objects with the matrix phase: } \\
\qquad \frac{\partial \Phi_{1,0}+\partial \Phi_{2,0}}{\Phi_{1}+\Phi_{2}+\Phi_{3}+\Phi_{4}}=! \mathrm{min} \\
\text { which will constrain the overall "symbol" to be a disc. } \\
\text { *note that the boundary also is an area and the ratio thus is dimensionless }\end{array}$ \\
\hline
\end{tabular}




\section{References}

1. Schmitz, G. J.: "Quantitative mereology: An essay to align physics laws with a philosophical concept" Physics Essays, 33, (2020) 479; http://dx.doi.org/10.4006/0836-1398-33.4.479

2. Casati, R. and Varzi, A. C. (1999): "Parts and Places”, MIT Press ; ISBN: 9780262517072

3. Whitehead, A. N.: Process and Reality, New York, MacMillan, 1929

4. Schulz, D. R.: "Platonism, Mereology and Whitehead's Process Ontology", Monash University 2015 (https://bridges.monash.edu/articles/thesis/Platonism_mereology_and_Whitehead_s_process_ontology/4719385)

5. E. Ghedini , G. Goldbeck, A. Hashibon, G. J. Schmitz, and J. Friis (2021): "EMMO: The Elementary Multiperspective Materials Ontology " available online at GitHub: https://github.com/emmo-repo/EMMO

6. Arp,R. , Smith,B. and Spear, A.D.: Building Ontologies with Basic Formal Ontology; MIT Press; ISBN: 9780262527811

7. https://en.wikipedia.org/wiki/BORO

8. $\quad$ https://en.wikipedia.org/wiki/Dublin_Core

9. Herre, H.; Heller, B.; Burek, P.; Hoehndorf, R.; Loebe, F. \& Michalek, H.: General Formal Ontology (GFO): A Foundational Ontology Integrating Objects and Processes. Part I: Basic Principles. Research Group Ontologies in Medicine (Onto-Med), University of Leipzig.

10. https://en.wikipedia.org/wiki/Cyc

11. https://en.wikipedia.org/wiki/Suggested_Upper_Merged_Ontology

12. https://en.wikipedia.org/wiki/UMBEL

13. Nardi, J. C., Falbo, R. D. A., Almeida, J. P. A., Guizzardi, G., Pires, L. F., van Sinderen, M. J., \& Guarino, N. (2013, September). "Towards a commitment-based reference ontology for services". In Enterprise Distributed Object Computing Conference (EDOC), 2013 17th IEEE International (pp. 175-184). IEEE.

14. Gangemi, A., Guarino, N., Masolo, C., Oltramari, A., Schneider, L., 2002. Sweetening Ontologies with DOLCE, in: Gómez-Pérez, A., Benjamins, V.R. (Eds.), Knowledge Engineering and Knowledge Management: Ontologies and the Semantic Web. Springer Berlin Heidelberg, Berlin, Heidelberg, pp. 166-181. https://doi.org/10.1007/3-540-45810-7_18

15. Borgo, S. and Masolo, C.: "Ontological Foundations of DOLCE" In book: Theory and Applications of Ontology: Computer Applications. Springer Science+Business Media B.V, New York, USA. https://dx.doi.org/10.1007/978-90-481-8847-5

16. https://en.wikipedia.org/wiki/Object_Process_Methodology

17. Dori, D.: Object-Process Methodology: A Holistic Systems Paradigm; Springer Science \& Business Media ISBN 3642562094, 9783642562099

18. Ivanova, T.: "Extended Contact Algebras and Internal Connectedness"; Studia Logica (2019) DOI: 10.1007/s11225-019-09845-6

19. de Laguna, T.: Point, line and surface as sets of solids, J. Philos, 19 (1922),449-461.

20. Borgo,S. and Masolo, C.: "Full Mereogeometries"; The Review of Symbolic Logic (2006) DOI: 10.1017/S1755020310000110

21. Borgo, S. , Ferrario, R., Masolo, C. and Oltramari, A.: "Mereogeometry and Pictorial Morphology" Image; topical issue 5 (February 2007)

22. Levesque, H. J. (ed.). “Readings in Knowledge Representation”. Morgan Kaufmann. ISBN 978-0-934613-01-9.

23. Stell, J.G.: Mereotopology and Computational Representations of the Body; Computational Culture, 6. ISSN 2047-2390

24. Randell, D. A., Cui, Z. and Cohn, A. G.: A spatial logic based on regions and connection. In: B. Nebel, W. Swartout, C. Rich (Eds.) Proceedings of the 3rd International Conference Knowledge Representation and Reasoning, Morgan Kaufmann, Los Allos, CA(1992)p 165.

25. de Vries, H.. Compact spaces and compactications, Van Gorcum, 1962

26. Stell, J.G.: Boolean connection algebras: A new approach to the Region Connection Calculus, Artif. Intell. 122 (2000), $111\{136$.

27. Vakarelov,D, Düntsch, I. and Bennett, B.: A note on proximity spaces and connection based mereology. In: Welty, C. and Smith, B., editors, Proceedings of the 2nd International Conference on Formal Ontology in Information Systems (FOIS'01), ACM, (2001), 139-150.

28. Vakarelov,D., Dimov, G. , Düntsch, I. and Bennett, B.: A proximity approach to some region based theory of space. Journal of applied non-classical logics, vol. 12, No3-4 (2002), 527-559

29. Bennett, B. and Düntsch, I.: Axioms, Algebras and Topology. In:Handbook of Spatial Logics, M. Aiello, I. Pratt, and J. van Benthem (Eds.), Springer,2007, 99-160.

30. Düntsch, I. and Winter, M.: A representation theorem for Boolean contact algebras. Theoretical Computer Science (B), 347 (2005), 498-512.

31. Dimov, G. and Vakarelov,D.: Contact algebras and region based theory of space: A proximity approach I. Fundamenta Informaticae, vol. 74, No 2-3,(2006) 209-249

32. Düntsch, I. and Vakarelov, D.: Region-based theory of discrete spaces: A proximity approach. In: Nadif, M., Napoli, A., SanJuan, E., and Sigayret, A. EDS, Proceedings of Fourth International Conference Journees del'informatique Messine, 123-129, Metz, France, 2003. Journal version in: Annals of Mathematics and Artificial Intelligence, 49(1-4):5-14, 2007.

33. Balbiani,P. and Ivanova, T.: "Representation theorems for extended contact algebras based on equivalence relations" Studia Logica · September 2020 DOI: 10.1007/s11225-020-09923-0 
34. Burch, R. (1991): “A Peircean Reduction Thesis: The Foundations of Topological Logic”, Texas Tech University Press, Lubbock, TX

35. Correia J.H., Pöschel R. (2006): “The Teridentity and Peircean Algebraic Logic” In: Schärfe H., Hitzler P., Øhrstrøm P. (eds) Conceptual Structures: Inspiration and Application. ICCS 2006. Lecture Notes in Computer Science, vol 4068. Springer, Berlin, Heidelberg. https://doi.org/10.1007/11787181_17

36. Vakarelov,D.: “A mereotopology based on sequent algebras", Journal of Applied Non-Classical Logics 27 (3-4):342-364 (2017)

37. Vakarelov,D. : “Extended Mereotopology Based on Sequent Algebras” Journal of Applied Non-Classical Logics · January 2018 DOI: $10.1080 / 11663081.2017 .1420590$

38. Grüniger M., Chui, C., Yi Ru, Jona Thai “A Mereology for Connected Structures” in: B. Brodaric and F. Neuhaus (Eds.)” Formal Ontology in Information Systems", IOS Press.Doi:10.3233/FAIA200670

39. Stell, J. G., West, M.: “A 4-Dimensionalist Mereotopology": https://www.researchgate.net/publication/249754393_A_4-Dimensionalist_Mereotopology

40. Vakarelov,D.: "Point-free theories of space and time" https://arxiv.org/abs/2004.14755v1

41. https://www.researchgate.net/project/Mereotopology-static-and-dynamic

42. Nenchev, V.: “Dynamic Realtional Mereotopology: Logics for stable and unstable relations" Logic and Logical Philosophy Volume 22 (2013), 295-325 DOI: 10.12775/LLP.2013.014

43. Galton, A.: "The mereotopology of discrete space", in: C. Freska, D.M. Mark (Eds.), Spatial Information Theory: Cognitive and Computational Foundations of Geographic Science, 1999, 251-266.

44. Galton, A.: “Discrete Mereotopology" in: Calosi C., Graziani P. (eds): “Mereology and the Sciences”. Synthese Library (Studies in Epistemology, Logic, Methodology, and Philosophy of Science), vol 371. Springer, Cham, 2014, 293-321.

45. Landini, G., Galton, A., Randell, D. \& Fouad, S. 2019: 'Novel applications of discrete mereotopology to mathematical morphology', Signal Processing: Image Communication, vol. 76, pp. 109-117. https://doi.org/10.1016/j.image.2019.04.018

46. Boolos, G.: "to be is to be the value of a variable (or to be some values of some variables)", Journal of Philosophy, 81(1984), 430-49

47. Schmitz, G.J., Böttger, B., Eiken, J., Apel, M. , Viardin, A., Carré, A. and Laschet, G.: "Phase-field based simulation of microstructure evolution in technical alloy grades" Int. J. Adv. Eng. Sci. Appl. Math. 24 (2012) 126 DOI 10.1007/s12572-011-0026-y

48. Eiken, J.: "A Phase-Field Model for Technical Alloy Solidification" PhD Thesis RWTH Aachen (2009) ISBN : 978-3-8322-9010-8

49. Böttger, B., Apel, M., Budnitzki, M., Eiken, J. , Laschet, G., Zhou, B.: "Calphad coupled phase-field model with mechano-chemical contributions and its application to rafting of $\gamma^{\prime}$ in CMSX-4" Computational Materials Science 184 (2020) 109909 https://doi.org/10.1016/j.commatsci.2020.109909

50. Hamaker, H.C.: The London-van der Waals Attraction between Spherical Particles. In: Physica. Bd. 4, Nr. 10, 1937, S. 10581072, doi:10.1016/S0031-8914(37)80203-7

51. Ginzburg, V.L. and Landau, L.D. : Zh. Eksp. Teor. Fiz. 20, 1064 (1950). English translation in: L. D. Landau, Collected papers (Oxford: Pergamon Press, 1965) p. 546

52. Cahn, J. W.; Hilliard, J. E. (1958). "Free Energy of a Nonuniform System. I. Interfacial Free Energy". The Journal of Chemical Physics. AIP Publishing. 28 (2): 258-267. doi:10.1063/1.1744102. ISSN 0021-9606.

53. Allen, S. M.; Cahn, J. W. (1976). "On Tricritical Points Resulting from the Intersection of Lines of Higher-Order Transitions with Spinodals". Scripta Metallurgica. 10 (5): 451-454. doi:10.1016/0036-9748(76)90171-x.

54. Kosterlitz, J. M.; Thouless, D. J.: Ordering, metastability and phase transitions in two-dimensional systems. In: Journal of Physics C: Solid State Physics. Band 6, Nr. 7, April 1973, S. 1181-1203, doi:10.1088/0022-3719/6/7/010

55. Langer, J. (1978): unpublished handwritten notes on the phase-field concept

56. Caginalp, G. An analysis of a phase field model of a free boundary. Arch. Rational Mech. Anal. 92, 205-245 (1986). https://doi.org/10.1007/BF00254827

57. Fix,G.: Phase field models for free boundary prolems. In: Free boundary problems Vol.II, (ed.A.Fasano,M.Primicerio) Piman Boston (1983)

58. Kobayashi, R.: "Modeling and numerical simulations of dendritic crystal growth" Physica D: Nonlinear Phenomena Vol. 63 34 (1993) 410

59. Steinbach, I. , Pezzolla, F., Nestler, B., Seeßelberg, M., Prieler, R., Schmitz, G.J. , Rezende, J.L.L.: „A phase field concept for multiphase systems" Physica D 94(1996), p.135-147

60. Böttger, B.; Eiken, J.; Apel, M.: "Multi-ternary extrapolation scheme for efficient coupling of thermodynamic data to a multi-phase-field model", Comput. Mater. Sci., 108, 283 (2015)

61. MICRESS: The MICRostructure Evolution Simulation Software: www.micress.de (accessed Feb 20th 2021)

62. OpenPhase: http://openphase-solutions.com/ (accessed June9th 2021)

63. FiPY : https://www.ctcms.nist.gov/fipy/(accessed June9th 2021)

64. Provatas, N.; Elder, K.: "Phase-Field Methods in Materials Science and Engineering". Wiley VCH, Weinheim DOI:10.1002/9783527631520

65. Steinbach, I. ( 2009): „Phase-field models in materials science”, Modelling Simul. Mater. Sci. Eng. 17073001

66. Grain growth video animation: available at https://www.youtube.com/watch?v=NIzsjGE-Q4Y

67. Weisstein, E. W. "Heaviside Step Function." From MathWorld--A Wolfram Web Resource. https://mathworld.wolfram.com/HeavisideStepFunction.html 
68. Schmitz, G. J.: “Thermodynamics of Diffuse Interfaces", in H. Emmerich, B. Nestler, M. Schreckenberg (eds): "Interface and Transport Dynamics" Springer Lecture Notes in Computational Science and engineering, ISBN 3-540-40367-1 (2003) p. 47-64

69. Schmitz, G.J.: “Entropy and Geometric Objects", Entropy 2018, 20(6), 453; https://doi.org/10.3390/e20060453

70. de Gennes, P.G..: "Wetting: statics and dynamics". Reviews of Modern Physics. 57 (1985) (3): 827-863. doi:10.1103/RevModPhys.57.827

71. Eiken, J.: Discussion of the Accuracy of the Multi-Phase-Field Approach to Simulate Grain Growth with Anisotropic Grain Boundary Properties, ISIJ 60 (2020) 8 , 1832; DOI https://doi.org/10.2355/60 isijinternational.ISIJINT-2019-722

72. Mulliken, R.S. Electronic Structures of Polyatomic Molecules and Valence I. Phys. Rev. 1932, 40, 55-62.

73. Banchetti-Robino, M.P.; Llored, J.-P.:"Reality Without Reification: Philosophy of Chemistry's Contribution to Philosophy of Mind" In Essays in the Philosophy of Chemistry; Scerri, E., Fisher, G., Eds.; Oxford University Press: Oxford, UK, 2016; pp. 83

74. Banchetti-Robino, M.P.: "The Limits of Classical Extensional Mereology for the Formalization of Whole-Parts Relations in Quantum Chemical Systems" Philosophies 2020, 5, 16; doi:10.3390/philosophies5030016

75. Foto by Andrew Vargas, reproduced under CC BY 2.0, https://commons.wikimedia.org/w/index.php?curid=12812037

76. moviehttp://www22.pair.com/csdc/download/spotsmovie.avi

77. Kiehn, R.M.:"Falaco Solitons, Cosmological strings in swimming pool" http://www22.pair.com/csdc/pdf/falaco85i.pdf

78. 3D connected moving vortices: movie available at https://www.youtube.com/watch?v=pnbJEg9r108

79. Te Vrugt, M: “Mereology of Thermodynamic Equilibrium” https://arxiv.org/abs/2104.11140v1 